\title{
THEORY OF NON-LINEAR SINGULAR DIFFERENTIAL SYSTEMS*
}

BY

W. J. TRJITZINSKY

1. Introduction. The most important published paper dealing with this subject is a recent work by the present author $\dagger$ in which further references to the literature of the subject will be found. A method, introduced for the first time by Trjitzinsky in $\left(\mathrm{T}_{1}\right)$, which is specifically applicable to non-linear problems lies at the basis of the developments in the present work. $\ddagger$

In this paper we consider the system of non-linear differential equations

$$
t^{-p} y_{j}(1)(t)=a_{j}\left(t, y_{1}, \cdots, y_{n}\right) \quad(j=1, \cdots, n ; p \text { an integer } \geqq 0), \S
$$

where

$$
\begin{aligned}
& a_{j}\left(t, y_{1}, \cdots, y_{n}\right)=l_{j}\left(t, y_{1}, \cdots, y_{n}\right)+q_{j}\left(t, y_{1}, \cdots, y_{n}\right), \\
& l_{j}\left(t, y_{1}, \cdots, y_{n}\right)=l_{1, j}(t) y_{1}+\cdots+l_{n, j}(t) y_{n}, \\
& q_{j}\left(t, y_{1}, \cdots, y_{n}\right)=\sum_{j} a_{i_{1}, i_{2}}, \cdots, i_{n}(t) y_{1}^{i_{1}} y_{2}^{i_{2}} \cdots y_{n}^{i_{n}} \\
&\left(i_{1}, i_{2}, \cdots, i_{n} \geqq 0 ; i_{1}+\cdots+i_{n} \geqq 2 ; j=1, \cdots, n\right) .
\end{aligned}
$$

The coefficients $l_{i, j}(t),{ }_{j} a_{i_{1}}, \cdots, i_{n}(t)$ are assumed to be analytic at $t=\infty$ for $|t| \geqq r(>0)$, while the series of the second member of (1.1b) are supposed to be convergent for

$$
|t| \geqq r ;\left|y_{1}\right|,\left|y_{2}\right|, \cdots,\left|y_{n}\right| \leqq \rho .
$$

Moreover, it will be assumed that the linear system obtained by letting $q_{j}\left(t, y_{1}, \cdots, y_{n}\right) \equiv 0(j=1, \cdots, n)$ is actually of order $n$, and that it is not of Fuchsian type (cf. \$2, italics preceding (2.6)).

The analytic theory will be developed for the complex neighborhood of the singular point $t=\infty$. The main results of this work are embodied in the theorems at the end of $\$ \S 7,9,10$, and 15 .

* Presented to the Society, September 9, 1937; received by the editors December 9, 1936.

$\dagger$ W. J. Trjitzinsky, Analytic theory of non-linear singular differential equations, Mémorial des Sciences Mathématiques, Paris. In the sequel this paper will be referred to as $\left(T_{1}\right)$.

$\ddagger$ This method has been also applied in the paper by W. J. Trjitzinsky, Non-linear difference equations, Compositio Mathematica, vol. 5 (1937), pp. 1-66.

$\S p$ is taken as small as is compatible with the stated hypotheses.

$\| \rho$ is taken sufficiently small so that the function represented by the series is analytic at every point of the specified region. 
The system (A) contains as special cases some of the important instances of dynamical differential systems. From the point of view of dynamical applications the real variable theory is important. However, in so far as the problems of the present paper are concerned our interest lies in the complex plane. A considerable variety of new situations of mathematical interest arises in the field now under consideration.

We shall also consider systems (B), obtained from (A) by assuming that the

$$
l_{i, j}(t), \quad{ }_{j} a_{i_{1}, i_{2}}, \cdots, i_{n}(t)
$$

are all independent of $t$ and that $p=0$. These systems are of importance in dynamics. Our concern here, as well as throughout the paper, is with the analytic character of solutions "in the small" and, as stated before, with the complex neighborhood of the singular point.

Finally, in $\$ \$ 11-15$ an investigation is given for systems (C) of the form

$$
\lambda^{-p} y_{j}{ }^{(1)}(x)=a_{j}\left(\lambda, x, y_{1}, \cdots, y_{n}\right) \quad(j=1, \cdots, n ; \text { integer } p \geqq 0)
$$

where $\lambda$ is a parameter and where the second members are analytic in $\lambda, y_{1}, \cdots, y_{n}$ at $\lambda=\infty, y_{1}=\cdots=y_{n}=0$, for $x$ on a real interval, and continuous in $x$ on this interval. The theory of such systems will be given for the neighborhood of the singular point $\lambda=\infty$. The precise formulation of this problem, together with some references, will be found in $\$ 11$.

2. Semi-formal aspects. Formal solutions of the system (A) will now be constructed. These will appear as series, in general divergent, whose coefficients are functions determined by a sequence of well-defined analytic processes. For these reasons the construction and consideration of these solutions can be referred to as the domain of "semi-formal aspects" of the theory of systems (A).

Consider now the linear system associated with (A),

$$
t^{-p} y_{i}^{(1)}(t)=l_{i}\left(t, y_{1}, \cdots, y_{n}\right) \quad(i=1, \cdots, n)
$$

(cf. (1.1a)). By hypothesis

$$
\left|\left(l_{i, j}(t)\right)\right| \not \equiv 0 . \dagger
$$

The general asymptotic theory of such systems has been given by Trjitzinsky. $\ddagger$ It will be assumed that the reader is acquainted with the main results

$\dagger A=\left(a_{i, j}\right)$ is to denote a matrix of $n^{2}$ elements $(i, j=1, \cdots, n)$ with the displayed element in the $i$ th row and $j$ th column. The symbol $\left|\left(a_{i, j}\right)\right|=|A|$ is to stand for the determinant of $A$. The inverse of the matrix $A$ will be denoted by $A^{-1}\left(=\left(a_{i, j}\right)^{-1}\right)$.

$\ddagger$ W. J. Trjitzinsk.y, Analytic theory of linear differential equations, Acta Mathematica, vol. 62 (1934), pp. 167-226. In the sequel this paper will be referred to as $\left(\mathrm{T}_{2}\right)$. 
and methods contained in $\left(\mathrm{T}_{2}\right)$.

The system (LA) can be written in matrix form as

$$
Y^{(1)}(t)=Y(t) A(t), \quad Y(t)=\left(y_{i, j}(t)\right),
$$

where

$$
A(t)=\left(t^{p} l_{i, j}(t)\right) \quad(i, j=1, \cdots, n) .
$$

The elements of a row in $Y(t)$ will constitute a solution of (LA). The system $\left(\mathrm{LA}_{1}\right)$ has a singularity of finite rank at $t=\infty$.

The following definitions will now be introduced.

Definition 1. Generically $\left\{t_{q}\right\}$ ( $q$ an integer $\geqq 0$ ) is to denote an expression

$$
\rho_{0}(t)+\rho_{1}(t) \log t+\cdots+\rho_{q}(t) \log ^{q} t,
$$

where $\rho_{j}(t)(j=0, \cdots, q)$ is a series, possibly divergent, of the form

$$
\rho_{j, 0}+\rho_{j, 1} t^{-1 / k}+\rho_{j, 2} t^{-2 / k}+\cdots
$$

(k a positive integer). $\{t\}^{*}$ is to stand for an expression $\{t\}_{q}$.

Definition 2. A curve $B$ will be said to be regular if it is analytic in every finite part and extends to infinity where it possesses a unique limiting direction. $\dagger$

Definition 3. A region $R$ will be said to be regular if it is closed, extends to infinity, and is such that for $t$ in $R|t| \geqq r_{1}(>0)$. The boundary of $R$ is to be simple and is to consist of an arc $\gamma$ of the circle $|t|=r_{1}$ and of two regular (cf. Definition 2) curves extending from different extremities of $\gamma$. Generically $R\left(\theta_{1}, \theta_{2}\right)$ is to denote a regular region for which the two regular curves (making part of the boundary) have limiting directions $\theta_{1}$ and $\theta_{2}$, respectively. The number $\left|\theta_{1}-\theta_{2}\right|$ will be termed the opening of $R$.

Definition 4. Generically $[t]_{q}$ is to denote a function of the form

$$
p_{0}(t)+p_{1}(t) \log t+\cdots+p_{q}(t) \log ^{q} t,
$$

where $p_{j}(t)(j=0, \cdots, q)$ is a function, analytic for $t \neq \infty$ in a regular (cf. Definition 3 ) region $R$, such that

$$
p_{j}(t) \sim \rho_{j}(t)
$$

$(t$ in $R)$,

where $\rho_{j}(t)$ is of the form (2.3). $\ddagger[t]^{*}$ is to stand for $[t]_{q}$.

$\dagger$ The implication is that a regular curve $\left(|t| \geqq r^{\prime}\right)$ is representable by an equation $R f(t)=0$, where $f(t)$ is analytic for $|t| \geqq r^{\prime}$, but may possess a singularity at $t=\infty$.

$\ddagger$ Unless otherwise stated asymptotic relations are asymptotic in the ordinary sense, i.e., to infinitely many terms. Thus, if $a(t) \sim a_{0}+a_{1} t^{-1 / k}+\cdots(t$ in a regular region $R)$ to infinitely many terms, one has $a(t)=a_{0}+\cdots+a_{n-1} t^{-(n-1) / k}+a_{n}(t) t^{-n / k}\left(\left|a_{n}(t)\right|<b_{n} ; t\right.$ in $\left.R\right)$ for $n=1,2, \cdots$. If $n$ cannot be increased beyond $n_{0}$ the above relation is to $n_{0}$ terms. 
One may write $[t]_{q} \sim\{t\}_{q}$ and $[t]^{*} \sim\{t\}^{*}(t$ in $R)$.

The system $\left(\mathrm{LA}_{1}\right)$ possesses a formal matrix solution $\dagger$

$$
S(t)=\left(s_{i, j}(t)\right)=\left(e^{Q_{i}(t)} t^{r_{i, j}} \sigma_{i, j}(t)\right),
$$

where

$$
Q_{i}(t)=\sum_{\nu=0}^{l_{i}-1} q_{\nu} i^{\left(t^{(} l_{i}-\nu\right) / k_{i}} \quad\left(\text { integer } k_{i} \geqq 1 ; i=1, \cdots, n ; l_{i} \geqq 1\right),
$$

the $r_{i, j}$ for a fixed $i$ may differ only by rational numbers, and

$$
\sigma_{i, j}(t)=\{t\}_{q}
$$

$$
(i, j=1, \cdots, n) . \ddagger
$$

Moreover, formally $|S(t)|$ does not vanish. $\S$

In consequence of a hypothesis made in $\$ 1$ the system (LA) is not of Fuchsian type; that is, not all of the polynomials $Q_{i}(t)(i=t, \cdots, n)$ involved in (2.5) are identically zero. We let $B_{i, j}$ denote a regular curve along which

$$
R\left(Q_{i}(t)-Q_{j}(t)\right)=0 .
$$

It will be understood that there are no $B_{i, j}$ curves corresponding to any pair of values $i, j$ for which $Q_{i}(t) \equiv Q_{j}(t)$. Let

$$
R_{1}^{\prime}, R_{2}^{\prime}, \cdots, R_{N}^{\prime}
$$

be regular regions (cf. Definition 3) separated by the $B_{i, j}$ curves (formed for all admissible pairs of values $i, j$ ) in such a way that interior to any such region there is no $B_{i, j}$ curve. Consider a particular region $R_{k}{ }^{\prime}$ of the set (2.6a). It is of the form $R\left(\theta_{k, 1}, \theta_{k, 2}\right)$ (cf. Definition 3) where, let us say, $\theta_{k, 1} \leqq \theta_{k, 2}$. Let $B_{k, l}^{\prime}$ and $B_{k, r}^{\prime}$ denote the regular curves forming part of the boundary of $R_{k}^{\prime}$ and possessing at infinity the limiting directions $\theta_{k, 1}$ and $\theta_{k, 2}$, respectively.

In view of the Fundamental Existence Theorem given in $\left(T_{2}\right)$ and in consequence of the connection between single $n$th order linear differential equations with systems, the following can be asserted for any fixed $k\left(1 \leqq k \leqq N^{\prime}\right)$. If $\theta_{k, 1}=\theta_{k, 2}$, the matrix equation $\left(\mathrm{LA}_{1}\right)$ ' has a matrix solution

$$
Y(t)=\left(y_{i, j}(t)\right) \quad(i, j=1, \cdots, n),
$$

whose elements $y_{i, j}(t)$ are analytic in $R_{k}^{\prime}$ for $t \neq \infty$, while

$$
Y(t) \sim S(t)
$$

$\left(t\right.$ in $\left.R_{k}^{\prime}\right)$.

$\dagger\left(\mathrm{T}_{2}\right)$, p. 171

$\ddagger$ It is seen that the $\sigma_{i, j}(t)$ can be so selected that the $r_{i, j}$ are independent of $j$.

$\S$ This implies that when $|S(t)|$ is formally computed as an expression of the type (exp. [polynomial in $\left.t^{\alpha}\right]$ ) $t^{\beta}\{t\}_{0}$ (cf. Definition 1), not all the coefficients in $\{t\}_{0}$ are zero. 
The implication of $(2.7)$ is

$$
y_{i, j}(t)=e^{Q_{i}(t)} t^{r_{i, j}}[t]_{q}
$$$$
\left(i, j=1, \cdots, n ; t \text { in } R_{k}^{\prime}\right)
$$

(cf. Definition 4). If $\theta_{k, 1}<\theta_{k, 2}$ there exist regular overlapping subregions of $R_{k}^{\prime}$,

$$
{ }_{r} R_{k}^{\prime}=R\left(\theta_{k, 1}, \theta_{k, 2}\right), \quad{ }_{l} R_{k}^{\prime}=R\left(\theta_{k, 1}, \theta_{k, 2}\right),
$$

whose boundaries contain $B_{k, l}^{\prime}$ and $B_{k, r}^{\prime}$, respectively. $\dagger$ The regions (2.8) can be so selected (depending on the polynomials $(2.5 \mathrm{a})$; for details cf. $\left(\mathrm{T}_{2}\right)$ ) that $\left(\mathrm{LA}_{1}\right)$ possesses two matrix solutions,

$$
{ }_{r} Y(t)=\left({ }_{r} y_{i, j}(t)\right), \quad{ }_{l} Y(t)=\left({ }_{l} y_{i, j}(t)\right),
$$

such that

$$
\begin{aligned}
& { }_{r} Y(t) \sim S(t) \\
& { }_{i} Y(t) \sim S(t)
\end{aligned}
$$$$
\left(t \text { in }{ }_{r} R_{k}^{\prime}\right) \text {, }
$$$$
\left(t \text { in }{ }_{l} R_{k}{ }^{\prime}\right) \text {. }
$$

Let $P(t)$ stand for a polynomial (2.5a) which is not identically zero. It is clear that one may find a regular region $R^{\prime}=R\left(\theta^{\prime}, \theta^{\prime \prime}\right)\left(\theta^{\prime}<\theta^{\prime \prime}\right)$ such that

$$
e^{P(t)} \sim 0
$$

$\left(t\right.$ in $\left.R^{\prime}\right)$.

Since not all of the $Q_{i}(t)$ are identically zero it is observed that there exist regions

$$
R_{1}, R_{2}, \cdots, R_{N}
$$

such that, if $R$ stands for a particular one of them, the following statements are true:

(i) $R$ is a regular region (cf. Definition 3 ) which is a subset of a region referred to in (2.7), (2.8b), (2.8c).

(ii) There exist polynomials of the set (2.5a), $Q_{i_{1}}(t), Q_{i_{2}}(t), \cdots, Q_{i_{m}}(t)$ $(1 \leqq m \leqq n)$ such that

$$
e^{Q_{i \nu}(t)} \sim 0 \quad(\nu=1,2, \cdots, m ; t \text { in } R) .
$$

As a matter of notation, which does not entail any loss of generality, the polynomials referred to in (2.9a) will be designated as

$$
Q_{1}(t), Q_{2}(t), \cdots, Q_{m}(t) \text {. }
$$

Moreover, without any loss of generality one may write

$$
R Q_{1}(t) \leqq R Q_{2}(t) \leqq \cdots \leqq R Q_{n}(t)
$$

$(t$ in $R)$.

† The other regular curve, which forms part of the boundary of ${ }_{r} R_{k}^{\prime}$ (or ${ }_{l} R_{k}^{\prime}$ ), is interior to $R_{k}^{\prime}$ and has at infinity the limiting direction of $B_{k, r}^{\prime}\left(\right.$ or $\left.B_{k, l}^{\prime}\right)$. 
In the sequel, unless stated otherwise, we confine ourselves to a particular region $R$ referred to in connection with (i), (ii), and (2.9b). In consequence of (i) there exists a matrix solution of $\left(\mathrm{LA}_{1}\right),{ }_{0} Y(t)=\left({ }_{0} y_{i, j}(t)\right)$, such that

$$
{ }_{0} Y(t) \sim S(t)
$$

$(t$ in $R)$.

We consider now a solution of (LA) of the form

$$
{ }_{1} y_{j}(t)=\sum_{\lambda=1}^{m} c_{\lambda} 0 y_{\lambda, j}(t) \quad(j=1, \cdots, n),
$$

where $c_{1}, c_{2}, \cdots, c_{m}$ are arbitrary constants. The non-linear system (A) of $\S 1$ will be formally satisfied by the series (in general divergent) of the form

$$
y_{j}(t)={ }_{1} y_{j}(t)+{ }_{2} y_{j}(t)+\cdots+{ }_{\nu} y_{j}(t)+\cdots \quad(j=1, \cdots, n) .
$$

Here the $y_{j}(t)(\nu=2,3, \cdots)$ will be determined in the sequel as certain functions analytic in $R(t \neq \infty)$ and of the form

$$
\begin{aligned}
{ }_{\nu} y_{j}(t)=\sum_{k_{1}, \cdots, k_{m}} c_{1}^{k_{1} c_{2}^{k_{2}} \cdots c_{m} c_{\nu} \eta_{k_{1}, k_{2}, \cdots, k_{m} ; j}(t)} \\
\quad\left(k_{1}, k_{2}, \cdots, k_{m} \geqq 0 ; k_{1}+k_{2}+\cdots+k_{m}=\nu\right) .
\end{aligned}
$$

The functions ${ }_{1} y_{j}(t)$ will be representable by (2.12a) with $\nu=1$ if we let

$$
{ }_{0} y_{\lambda, j}(t)={ }_{1} \eta_{k_{1}, k_{2}, \cdots, k_{m} ; j}(t) \quad(\lambda=1, \cdots, m ; j=1, \cdots, n),
$$

where $k_{\lambda}=1$ and $k_{i}=0$ for $i \neq \lambda$.

To determine the terms of the series (2.12) these series are substituted in (A). If we take account of (1.1), (1.1a), (1.1b), it follows that, since ${ }_{1} y_{j}(t)(j=1, \cdots, n)$ is a solution of (LA), we have

$$
\sum_{\nu=2}^{\infty}{ }_{\nu} y_{j}{ }^{(1)}(t)-t^{p} \sum_{\nu=2}^{\infty} l_{j}\left(t,{ }_{\nu} y_{1}, \cdots,{ }_{\nu} y_{n}\right)=t^{p} q_{j} \quad(j=1, \cdots, n),
$$

where

$$
\begin{aligned}
q_{j}=\sum_{i_{1}, \cdots, i_{n}} a_{i_{1}, \cdots, i_{n}}(t) \prod_{\alpha=1}^{n}\left[\sum_{\nu=1}^{\infty}{ }_{\nu} y_{\alpha}(t)\right]^{i_{\alpha}} \\
\left(i_{1}, \cdots, i_{n} \geqq 0 ; i_{1}+\cdots+i_{n} \geqq 2\right) .
\end{aligned}
$$

The first member in (2.13) may be written in the form

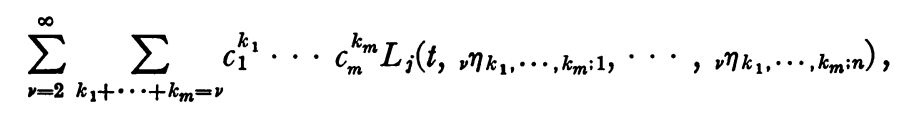

where 


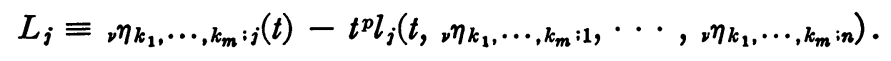

We now proceed to derive a formal expansion of $q_{j}$ according to powers of $c_{1}, \cdots, c_{m}$. First it is noted that, with $i_{\alpha}>0$,

$$
\begin{aligned}
{\left[\sum_{\nu=1}^{\infty}{ }_{\nu} y_{\alpha}(t)\right]^{i_{\alpha}} } & =\sum_{v_{1}, \cdots, v_{i_{\alpha}}=1} v_{1} y_{\alpha} v_{2} y_{\alpha} \cdots v_{v_{\alpha}} y_{\alpha} \\
& =\sum_{\gamma=i_{\alpha}}^{\infty} \sum_{\nu_{1}+\cdots+v_{i \alpha}=\gamma}{ }_{\nu_{1}} y_{\alpha} v_{2} y_{\alpha} \cdots v_{i_{\alpha}} y_{\alpha}\left(1 \leqq \nu_{1}, \nu_{2}, \cdots\right) .
\end{aligned}
$$

In view of (2.12a) it is observed that $\dagger$

$$
\begin{aligned}
& \prod_{\beta=1}^{i_{\alpha}}{ }_{\nu_{\beta}} y_{\alpha}=\prod_{\beta=1}^{i_{\alpha}} \sum_{k_{1}^{\beta}+\cdots+k_{m}{ }^{\beta}=\nu_{\beta}} c_{1}^{k_{1}^{\beta}} c_{2}^{k_{2}^{\beta}} \cdots c_{m}^{k_{m}{ }^{\beta}}{ }_{\nu_{\beta}} \eta_{k_{1}}^{\beta}, \cdots, k_{m}^{\beta} ; \alpha
\end{aligned}
$$

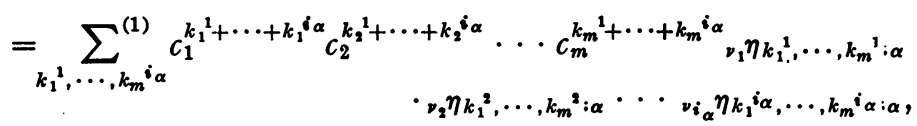

the summation symbol of the last member above being specified as follows:

$$
\begin{aligned}
& \sum^{(1)}=\sum\left(k_{i}{ }^{\beta} \geqq 0 ; k_{1}{ }^{1}+k_{2}{ }^{1}+\cdots+k_{m}{ }^{1}=\nu_{1} ;\right. \\
&\left.k_{1}{ }^{2}+k_{2}{ }^{2}+\cdots+k_{m}{ }^{2}=\nu_{2} ; \cdots ; k_{1}^{i \alpha}+k_{2}^{i \alpha}+\cdots+k_{m}^{i \alpha}=\nu_{i \alpha}\right) .
\end{aligned}
$$

It is next inferred that

$$
\prod_{\beta=1}^{i_{\alpha}}{ }_{\nu \beta} y_{\alpha}=\sum_{\delta_{1}+\cdots+\delta_{m}=\gamma} c_{1}^{\delta_{1}} c_{2}^{\delta_{2}} \cdots c_{m}^{\delta_{m}}{ }_{\gamma} \phi_{\nu_{1}, \nu_{2}, \cdots, \nu_{i \alpha} ; \alpha}^{\delta_{1}, \delta_{2}, \cdots, \delta_{m} ; i \alpha}
$$

where

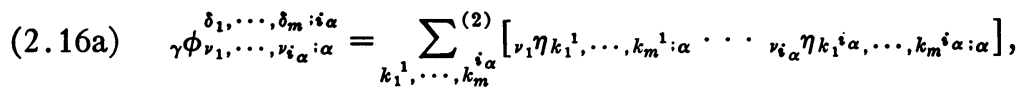

$$
\sum^{(2)}=\sum\left[k_{1}^{1}+\cdots+k_{1}^{i_{\alpha}}=\delta_{1} ; k_{2}^{1}+\cdots+k_{2}^{i_{\alpha}}=\delta_{2} ; \cdots ;\right.
$$

(2.16b) $k_{m}{ }^{1}+\cdots+k_{m}^{i_{\alpha}}=\delta_{m} ; k_{1}{ }^{1}+\cdots+k_{m}^{1}=\nu_{1} ; k_{1}{ }^{2}+\cdots+k_{m}{ }^{2}=\nu_{2} ; \cdots$;

$$
\left.k_{1}^{i \alpha}+\cdots+k_{m}^{i \alpha}=\nu_{i_{\alpha}}\right] \text {. }
$$

Substitution of (2.16) into (2.15) will yield

$$
\left[\sum_{\nu=1}^{\infty}{ }_{\nu} y_{\alpha}(t)\right]^{i_{\alpha}}=\sum_{\gamma=i \alpha}^{\infty} \sum_{\delta_{1}+\cdots+\delta_{m}=\gamma} c_{1}^{\delta_{1} \delta_{2}^{\delta_{2}}} \cdots c_{m}^{\delta_{m}} \psi_{\alpha}^{\delta_{1}, \delta_{2}, \cdots, \delta_{m}: i_{\alpha}}
$$

$\dagger$ Here the superscripts attached to the $k_{i}$ do not indicate powers. 
with

$$
{ }_{\gamma} \psi_{\alpha}^{\delta_{1}, \cdots, \delta_{m}: i \alpha}=\sum_{v_{1}+\cdots+v_{i_{\alpha}}=\gamma}{ }_{\gamma} \phi_{\nu_{1}, \cdots, i_{i_{\alpha}} ; \alpha}^{\delta_{1}, \cdots, \delta_{m} ; i_{\alpha}}
$$

It will be convenient to introduce the notation

$$
\eta_{\gamma}^{\alpha: i_{\alpha}}=\sum_{\delta_{1}+\cdots+\delta_{m}=\gamma} c_{1}^{\delta_{1}} \cdots c_{m}^{\delta_{m}} \psi_{\alpha}^{\delta_{1}, \cdots, \delta_{m}: i_{\alpha}}
$$

We may then write

$$
\begin{aligned}
\prod_{\alpha=1}^{n}\left[\sum_{\nu=1}^{\infty}{ }_{\nu} y_{\alpha}(t)\right]^{i_{\alpha}}=\prod_{\alpha=1}^{n} \sum_{\gamma_{\alpha=i_{\alpha}}}^{\infty} \eta_{\gamma_{\alpha}}^{\alpha: i_{\alpha}}=\sum_{\gamma_{1}, \cdots, \gamma_{n}} \prod_{q=1}^{n} \eta_{\gamma_{q}}^{q: i_{q}} \\
\left(\gamma_{1} \geqq i_{1}, \gamma_{2} \geqq i_{2}, \cdots, \gamma_{n} \geqq i_{n}\right) .
\end{aligned}
$$

In (2.18) the terms can be grouped so that

$$
\begin{aligned}
\prod_{\alpha=1}^{n}\left[\sum_{v=1}^{\infty}{ }_{\nu} y_{\alpha}(t)\right]^{i_{\alpha}}=\sum_{H=i_{1}+\cdots+i_{n}}^{\infty} \sum_{\gamma_{1}+\cdots+\gamma_{n}=H} \prod_{q=1}^{n} \eta_{\gamma_{q}}^{q: i q} \\
\left(\gamma_{1} \geqq i_{1}, \cdots, \gamma_{n} \geqq i_{n}\right) .
\end{aligned}
$$

Before we proceed further, the product involved in the second member of (2.18a) will be expanded. In view of $(2.17 \mathrm{~b})$

$$
\begin{aligned}
& \prod_{q=1}^{n} \eta_{\eta_{q q}}^{q: i_{q}}=\prod_{q=1}^{n} \sum_{q \delta_{1}+\cdots+q \delta_{m}=\gamma q} c_{1}^{q \delta_{1}} \cdots c_{m}^{q \delta_{m}}{ }_{\gamma_{q}} \psi_{q}^{q \delta_{1}} \cdots{ }_{q} \delta_{m}: i_{q} \\
& =\sum_{{ }_{1} \delta_{1}, \cdots, n \delta_{m}} c_{1}^{1^{1 \delta_{1}+\cdots+n \delta_{1}}} c_{2}^{1 \delta_{2}+\cdots+n \delta_{2}} \cdots c_{m}^{{ }^{1} \delta_{m}+\cdots+{ }_{n} \delta_{m}} \\
& \cdot \gamma_{1} \psi_{1}^{{ }^{1 \delta_{1}}, \cdots{ }_{1} \delta_{m}: i_{1}}{ }_{\gamma_{2}} \psi_{2}^{2_{1}^{\delta_{1}}, \cdots,{ }_{2} \delta_{m}: i_{2}} \cdots{ }_{\gamma_{n}} \psi_{n}^{{ }^{\delta_{1}}, \cdots{ }_{n} \delta_{m} ; i_{n}} \\
& \left({ }_{1} \delta_{1}+\cdots+{ }_{1} \delta_{m}=\gamma_{1} ;{ }_{2} \delta_{1}+\cdots+{ }_{2} \delta_{m}=\gamma_{2} ; \cdots ;{ }_{n} \delta_{1}+\cdots+{ }_{n} \delta_{m}=\gamma_{n}\right) .
\end{aligned}
$$

Substitute ${ }_{1} \delta_{q}+_{2} \delta_{q}+\cdots+{ }_{n} \delta_{q}=h_{q}(q=1,2, \cdots, m)$ in (2.19). Then we have

$$
\prod_{q=1}^{n} \eta_{\gamma q}^{q: i q}=\sum_{h_{1}, \cdots, h_{m}} c_{1}^{h_{1}} \cdots c_{m}^{h_{m}} \sum_{{ }_{1} \delta_{1}, \cdots, n}^{(3)}{ }_{\gamma_{1}} \psi_{1}^{1 \delta_{1}, \cdots, \delta_{m}: i_{1}} \cdots{ }_{\gamma n} \psi_{n}^{n_{1}^{\delta_{1}}, \cdots, n_{m} \delta_{m} ; i_{n}}
$$

where

$$
\begin{aligned}
\sum^{(3)}=\sum\left[{ }_{1} \delta_{q}+\cdots+{ }_{n} \delta_{q}=h_{q}(q=1, \cdots, m)\right. \\
\left.{ }_{1} \delta_{1}+\cdots+{ }_{1} \delta_{m}=\gamma_{1} ; \cdots ;{ }_{n} \delta_{1}+\cdots+{ }_{n} \delta_{m}=\gamma_{n}\right] .
\end{aligned}
$$

Now, as indicated in (2.18a), $\gamma_{1}+\cdots+\gamma_{n}=\pi$. Hence in (2.19a)

$$
\sum_{q=1}^{m} h_{q}=\sum_{q=1}^{m} \sum_{i=1}^{n} \delta_{q}=\sum_{i=1}^{n} \gamma_{i}=B \text {. }
$$


We shall write

$$
\sum_{{ }_{1} \delta_{1}, \cdots,{ }_{n} \delta_{m}}^{(3)} \cdots={ }_{H} \Lambda_{\gamma_{1}, \cdots, \gamma_{n}}^{h_{1}, \cdots, h_{m}: i_{1}, \cdots, i_{n}}
$$

Then (2.19a) will assume the form

$$
\prod_{q=1}^{n} \eta_{\gamma_{q}}^{q: i q}=\sum_{h_{1}+\cdots+h_{m}=H} c_{1}^{h_{1}} \cdots c_{m}^{h_{m}} \Lambda_{\gamma_{1}, \cdots, \gamma_{n}}^{h_{1}, \cdots, h_{m} ; i_{1}, \cdots, i_{n}} .
$$

Substitution of (2.20) into (2.18a) will yield

(2.21) $\prod_{\alpha=1}^{n}\left[\sum_{\nu=1}^{\infty}{ }_{\nu} y_{\alpha}(t)\right]^{i_{\alpha}}=\sum_{H=i_{1}+\cdots+i_{n}}^{\infty} \sum_{h_{1}+\cdots+h_{m}=H} c_{1}^{h_{1}} \cdots c_{m}^{h_{m}{ }_{H} \Lambda_{i_{1}}^{h_{1}}, \cdots, i_{n}}{ }^{h_{1}, \cdots, h_{m}}$,

where

(2.21a) ${ }_{H} \Lambda_{i_{1}, \cdots, i_{n}}^{h_{1}, \cdots, h_{m}}=\sum_{\gamma_{1}+\cdots+\gamma_{n}=H}{ }_{H} \Lambda_{\gamma_{1}, \cdots, \gamma_{n}}^{h_{1}, \cdots, h_{m} ; i_{1}, \cdots, i_{n}} \quad\left(\gamma_{1} \geqq i_{1}, \cdots, \gamma_{n} \geqq i_{n}\right)$.

Substitution of (2.21) in (2.13a) will result in

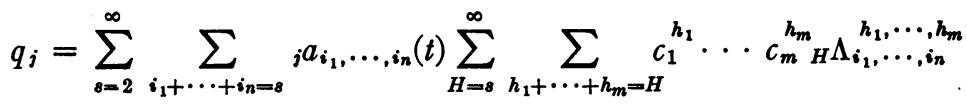

$$
\begin{aligned}
& =\sum_{s=2}^{\infty} \sum_{H=8}^{\infty} \sum_{h_{1}+\cdots+h_{m}=H} c_{1}^{h_{1}} \cdots c_{m}^{h_{m}} \Lambda_{s: j}^{h_{1}, \cdots, h_{m}}
\end{aligned}
$$

where

$$
{ }_{H} \Lambda_{8: j}^{h_{1}, \cdots, h_{m}}=\sum_{i_{1}+\cdots+i_{n=8}}{ }_{j} a_{i_{1}, \cdots, i_{n} H} \Lambda_{i_{1}, \cdots, i_{n}}^{h_{1}, \cdots, h_{m}} .
$$

If we denote the expression following the second summation symbol of the last member of (2.22) by $o_{s, H ; j}$, it is inferred that

$$
q_{j}=\sum_{s=2}^{\infty} \sum_{H=s}^{\infty} o_{s, H: j}=\sum_{H=2}^{\infty} \sum_{s=2}^{H} o_{s, H: j}
$$

By substituting the expression for $o_{s, H ; j}$ in (2.23) one obtains

$$
q_{j}=\sum_{H=2}^{\infty} \sum_{h_{1}+\cdots+h_{m}=H} c_{1}^{h_{1}} \cdots c_{m}^{h_{m}} \Gamma_{j}^{h_{1}, \cdots, h_{m}}
$$

with

$$
{ }_{H} \Gamma_{j}^{h_{1}, \cdots, h_{m}}=\sum_{s=2}^{H}{ }_{H} \Lambda_{s ; j}^{h_{1}, \cdots, h_{m}} .
$$

In consequence of the italicized statement following (2.13a) and in view of (2.23a) it is inferred from (2.13) that 


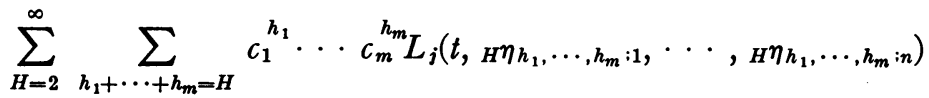

$$
\begin{aligned}
& =\sum_{H=2}^{\infty} \sum_{h_{1}+\cdots+h_{m}=H} c_{1}^{h_{1}} \cdots c_{m}^{h_{m}} t^{p}{ }_{H} \Gamma_{j}^{h_{1}, \cdots, h_{m}} .
\end{aligned}
$$

Thus the following set of differential equations is obtained:

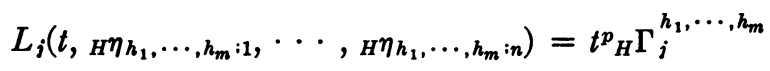

$$
\begin{aligned}
& \left(j=1,2, \cdots, n ; H=h_{1}+\cdots+h_{m} ; h_{1} \geqq 0, \cdots, h_{m} \geqq 0\right)
\end{aligned}
$$

(cf. (2.14a)). There exist systems $\left(S_{H}\right)$ for $H=2,3, \cdots$. It will be shown that the systems $\left(S_{2}\right),\left(S_{3}\right), \cdots$ can be solved in succession in order to determine all the ${ }_{\nu} \eta_{k_{1}}, \ldots, k_{m} ; i$ involved in the formal solutions (2.12) of the system (A), $\$ 1$ (cf. (2.12a)).

We shall now establish in detail the nature of the dependence of the second members of $\left(S_{H}\right)$ on the ${ }_{\nu} \eta_{k_{1}}, \ldots, k_{m} ; j$. Let

$$
\sum^{(4)}=\sum_{s=2}^{H} \sum_{i_{1}+\cdots+i_{n=8}} \sum_{\gamma_{1}+\cdots+\gamma_{n=H}} \sum_{1_{1}, \cdots n_{n} \delta_{m}}^{(3)} \quad\left(\gamma_{1} \geqq i_{1}, \cdots, \gamma_{n} \geqq i_{n}\right)
$$

(cf. (2.19b)). Here $s \leqq B$. For if $s>_{B}$ it would follow that $\gamma_{1}+\cdots+\gamma_{n} \geqq i_{1}+$ $\cdots+i_{n}=s>_{B}$, which would be contrary to the equality $\gamma_{1}+\cdots+\gamma_{n}=\boldsymbol{H}$.

Consecutive application of (2.23b), (2.22a), (2.21a), and (2.19c) will yield

$$
\begin{aligned}
{ }_{H} \Gamma_{j}^{h_{1}, \cdots, h_{m}} & =\sum_{s=2}^{H}{ }_{H} \Lambda_{s ; j}^{h_{1}, \cdots, h_{m}} \\
& =\sum_{s=2}^{H} \sum_{i_{1}+\cdots+i_{n=8}}{ }_{j} a_{i_{1}, \cdots, i_{n}{ }_{H}} \Lambda_{i_{1}, \cdots, i_{n}}^{h_{1}, \cdots, h_{m}} \\
& =\sum_{s=2}^{H} \sum_{i_{1}+\cdots+i_{n}=8}{ }_{j} a_{i_{1}, \cdots, i_{n}} \sum_{\gamma_{1}+\cdots+\gamma_{n}=H}{ }_{H} \Lambda_{\gamma_{1}, \cdots, \gamma_{n}}^{h_{1}, \cdots, h_{m} ; i_{1}, \cdots, i_{n}} \\
& =\sum^{(4)} a_{i_{1}, \cdots, i_{n}} \prod_{\alpha=1}^{n}{ }_{\gamma_{\alpha}} \psi_{\alpha}^{\alpha^{\delta_{1}}, \cdots, \alpha \delta_{m} ; i_{\alpha}} .
\end{aligned}
$$

In consequence of (2.17a) and (2.16a) it is finally deduced that

$$
\begin{aligned}
& { }_{H} \Gamma_{j}^{h_{1}, \cdots, h_{m}}=\sum^{(4)}{ }_{j} a_{i_{1}, \cdots, i_{n}} \prod_{\alpha=1}^{n} \sum_{\nu_{1}+\cdots+v_{i_{\alpha}}=\gamma_{\alpha}}{ }_{\gamma_{\alpha}} \phi_{\nu_{1}, \cdots, \nu_{i_{\alpha} ; \alpha}{ }^{\delta_{1}}, \cdots, \delta_{m} ; i \alpha} \\
& =\sum^{(4)} a_{i_{1}, \cdots, i_{n}} \prod_{\alpha=1}^{n} \sum_{\nu_{1}+\cdots+v_{i_{\alpha}}=\gamma_{\alpha}} \sum_{k_{1}{ }^{1}, \cdots, k_{m}{ }^{{ }^{\alpha} \alpha}}^{(2)} \prod_{r=1}^{i_{\alpha}}{ }_{\nu_{r} \eta_{k_{1}}{ }^{r}, \cdots, k_{m}{ }^{r} ; \alpha} \\
& \left(\boldsymbol{B}=h_{1}+\cdots+h_{m}\right)
\end{aligned}
$$


(cf. (2.24), (2.16b)). $\dagger$ In (2.26) the expression displayed after the product symbol with respect to $\alpha(\alpha=1, \cdots, n)$ is to be replaced by unity for every $\alpha$ for which $i_{\alpha}=0 ;$ moreover, for every such $\alpha$ we have $\gamma_{\alpha}=0$.

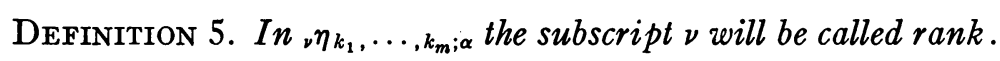

It will be now established that ${ }_{H} \Gamma_{j}^{h_{1}, \cdots, h_{m}}$ is independent of the ${ }_{\nu} \eta_{k_{1}}, \cdots, k_{m} ; \alpha$ of rank $\geqq H$. To prove this one notes that, in view of $(2.26)$, the ranks of the

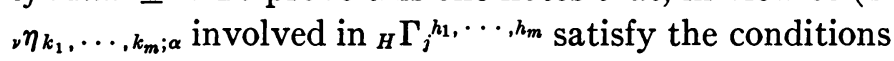

(2.27) $\nu_{1}+\cdots+\nu_{i \alpha}=\gamma_{\alpha} \geqq i_{\alpha}(\alpha=1,2, \cdots, n) ; \nu_{k} \geqq 1(k=1,2, \cdots)$; (2.27a) $\gamma_{1}+\cdots+\gamma_{n}=B ; i_{1}+\cdots+i_{n} \geqq 2 ; i_{k} \geqq 0 \quad(k=1, \cdots, n)$.

Thus

$$
\left(\nu_{1}+\cdots+\nu_{i_{1}}\right)+\left(\nu_{1}+\cdots+\nu_{i_{2}}\right)+\cdots+\left(\nu_{1}+\cdots+\nu_{i_{n}}\right)={ }_{H}
$$

The number of terms in the first member here is $i_{1}+\cdots+i_{n}$. If we had a $\nu_{\rho} \geqq B$ the left member in $(2.27 \mathrm{~b})$ would certainly be $\geqq B+1$ since there are at least two terms (we have $i_{1}+\cdots+i_{n} \geqq 2$ ) in this member, while all the $\nu_{k}$ are $\geqq 1$. This makes the truth of the italicized statement subsequent to Definition 5 evident.

The $\eta$ 's of rank one are known; they are the functions defined by (2.12b) where the ${ }_{0} y_{i, j}(t)$ are elements of the matrix solution ${ }_{0} Y(t)=\left({ }_{0} y_{i, j}(t)\right)$ of (LA). It is to be noted that in $R,{ }_{0} Y(t)$ satisfies $(2.10)$. The ${ }_{2} \Gamma_{j} h_{1}, \cdots, h_{m}$ $\left(h_{1}+\cdots+h_{m}=2 ; h_{1} \geqq 0, \cdots, h_{m} \geqq 0 ; j=1, \cdots, n\right)$ can accordingly be determined as functions of $t$. One may then determine all the $\eta$ 's of rank two with the aid of the system $\left(S_{2}\right)$. In general, if the $\eta$ 's of ranks $1,2, \cdots,{ }-1$ are known, the ${ }_{H} \Gamma_{j}{ }^{h_{1}}, \cdots, h_{m}$ may be computed as functions of $t$ and the $\eta$ 's of rank $B$ may be then constructed as solutions of the system $\left(S_{H}\right)$. Thus a mechanism has been set up for determining the functions ${ }_{\nu} y_{j}(t)$ of $(2.12 \mathrm{a})$; i.e., a device for computation of consecutive terms of the formal solution $\left(y_{1}(t), \cdots, y_{n}(t)\right)$ (cf. (2.12)) of the system (A) of $\$ 1$.

A matrix equation

$$
Y^{(1)}(t)=Y(t) A(t)+G(t) \quad\left(Y(t)=\left(y_{i, j}(t)\right) ; \quad G(t)=\left(g_{i, j}(t)\right)\right)
$$

is satisfied by

$$
Y(t)=C(t)_{0} Y(t) ; \quad C(t)=\int^{t} G(\tau)_{0} Y^{-1}(\tau) d \tau=\left(c_{i, j}(\tau)\right),
$$

where ${ }_{0} Y(t)=\left({ }_{0} y_{i, j}(t)\right.$ is a matrix solution of

$$
{ }_{0} Y^{(1)}(t)={ }_{0} Y(t) A(t) .
$$

$\dagger \operatorname{In}(2.16 \mathrm{~b})$ let $\delta_{i}={ }_{\alpha} \delta_{i}$ and $\gamma=\gamma_{\alpha}$. 
If the $g_{i, j}(t)=g_{j}(t)$ are independent of $i$, it is not difficult to verify that the $c_{i, j}(t)=c_{j}(t)$ are independent of $i$. The same will be true of the

$$
y_{i, j}(t)=y_{j}(t)=\sum_{\lambda=1}^{n} c_{\lambda}(t)_{0} y_{\lambda, j}(t) .
$$

By writing

$$
A(t)=\left(t^{p} l_{i, j}(t)\right) ; g_{i, j}(t)=g_{j}(t)=t_{H}^{p} \Gamma_{j}^{h_{1}, \cdots, h_{m}}(t),
$$

we accordingly infer that the system $\left(S_{H}\right)$ is satisfied with the aid of the formulas

$$
{ }_{H} \eta_{h_{1}}, \cdots, h_{m} ; j(t)=\sum_{\lambda=1}^{n} c_{\lambda}(t)_{0} y_{\lambda, j}(t)
$$

where

$$
\begin{aligned}
& c_{\lambda}(t)=c_{\lambda}\left(t ;{ }_{H} \Gamma_{1}^{h_{1}, \cdots, h_{m}}, \cdots,{ }_{H} \Gamma_{n}^{h_{1}, \cdots, h_{m}}\right) \\
& =\sum_{\lambda_{1}=1}^{n} \int^{t} \tau^{p}{ }_{H} \Gamma_{\lambda_{1}}^{h_{1}, \cdots, h_{m}}(\tau)_{0} \bar{y}_{\lambda_{1}, \lambda}(\tau) d \tau \\
& \left(j=1, \cdots, n ; h_{1}+\cdots+h_{m}=H ; h_{1} \geqq 0, \cdots, h_{m} \geqq 0\right) .
\end{aligned}
$$

In $(2.30 \mathrm{a})$ the ${ }_{0} \bar{y}_{i, j}(\tau)$ are the elements of the inverse of the matrix $\left({ }_{0} y_{i, j}(\tau)\right)$; that is,

$$
\left({ }_{0} \bar{y}_{i, j}(\tau)\right)=\left({ }_{0} y_{i, j}(\tau)\right)^{-1} .
$$

The above may be summarized as follows.

LEMMA 1. Let $R$ be a region referred to in connection with (i), (ii) (subsequent to (2.9)) and (2.9b). Let ${ }_{0} Y(t)$ be the matrix involved in (2.10). The system (A), $\$ 1$ is formally satisfied by series (2.12) for $j=1, \cdots, n$, in general divergent. The first term, ${ }_{1} y_{j}(t)$, in such a series is defined by (2.11). The subsequent terms ${ }_{\nu} y_{j}(t)$ are of the form (2.12a) with $\nu=2,3, \cdots$. The coefficients ${ }_{\nu} \eta_{k_{1}}, \ldots, k_{m} ; j(t)$ involved in ${ }_{\nu} y_{j}(t)$ can be determined in succession with the aid of (2.30), (2.30a), (2.30b), and (2.26). (In connection with (2.26) cf. italics subsequent to (2.26) and Definition 5.)

Note. $R$ can be replaced by a more general region. However, we restrict ourselves to regions of the stated kind, inasmuch as only corresponding to such regions will "actual" solutions of $(\mathrm{A}), \S 1$ be obtained.

3. Preliminaries to integration. It is to be noted that

$$
{ }_{0} y_{\lambda, \alpha}(t)=e^{Q_{\lambda}(t)} t^{r_{\lambda}}[t]_{q} \quad(t \text { in } R ; \lambda, \alpha=1, \cdots, n) .
$$


Thus the elements of the matrix (2.30b) are of the form

$$
{ }_{0} \bar{y}_{\lambda_{1}, \lambda}(\tau)=e^{-Q_{\lambda}(\tau)} \tau^{-\lambda_{\lambda}+\omega}[\tau]^{*} \quad\left(\omega \text { rational; } \tau \text { in } R ; \lambda_{1}, \lambda=1, \cdots, n\right)
$$

(cf. Definition $4, \S 2)$ ). In view of the notation (2.12a), we have

$$
\begin{aligned}
& { }_{1} \eta_{k_{1}{ }^{r}, \cdots, k_{m^{r}} ; \alpha}(\tau)=\exp \left[k_{1}{ }^{r} Q_{1}(\tau)+\cdots+k_{m^{r}} Q_{m}(\tau)\right] t^{k_{1}{ }^{r} r_{1}+\cdots+k_{m}{ }^{r} r_{m}}[t]_{q} \\
& \left(k_{1}^{r}+\cdots+k_{m}^{r}=1 ; k_{i}^{r} \geqq 0 ; \alpha=1, \cdots, n ; \tau \text { in } R\right) .
\end{aligned}
$$

In consequence of (2.26),

$$
{ }_{2} \Gamma_{j}{ }^{h_{1}, \ldots, h_{m}}(\tau)=\sum^{(4)} a_{i_{1}, \cdots, i_{n}}(\tau) \prod_{\alpha=1}^{n} \sum_{\nu_{1}+\cdots+\nu_{i_{\alpha}}=i_{\alpha}} \sum^{(2)} \prod_{r=1}^{i_{\alpha}}{ }_{\nu_{r}} \eta_{k_{1}, \cdots, k_{m}{ }^{r} ; \alpha}(\tau)
$$

With the aid of (2.16b) and (3.2) one obtains

$$
\begin{aligned}
& \prod_{r=1}^{i_{\alpha}}{ }_{{ }_{r}} \eta_{k_{1}{ }^{r}, \cdots, k_{m}{ }^{r}: \alpha}(\tau)=\exp \left[\left(k_{1}{ }^{1}+\cdots+k_{1}^{i_{\alpha}}\right) Q_{1}+\cdots\right. \\
& \left.+\left(k_{m}^{1}+\cdots+k_{m}^{i \alpha}\right) Q_{m}\right] t^{\left(k_{1}^{1}+\cdots+k_{1}^{i \alpha}\right) r_{1}+\cdots+\left(k_{m}{ }^{1}+\cdots+k_{m}{ }^{i} \alpha\right) r_{m}} \cdot[\tau] * \\
& =\exp \left[\delta_{1} Q_{1}+\cdots+\delta_{m} Q_{m}\right] \tau^{\delta_{1} r_{1}+\cdots+\delta_{m} r_{m}}[\tau]^{*} .
\end{aligned}
$$

Application of the summation

$$
\sum_{\nu_{1}+\cdots+v_{i_{\alpha}}=i_{\alpha}} \sum^{(2)}
$$

(cf. (2.16b) with $\delta_{i}={ }_{\alpha} \delta_{i}$ ) to the left member of (3.3a) will yield the function (3.3b) $\quad F_{\alpha}^{1}=$ generic form of the last member of (3.3a).

With the aid of (2.24) and (2.19b) it is inferred that

$$
\begin{aligned}
\prod_{\alpha=1}^{n} F_{\alpha}^{1}= & \exp \left[\left({ }_{1} \delta_{1}+\cdots+{ }_{n} \delta_{1}\right) Q_{1}+\cdots+\left({ }_{1} \delta_{m}+\cdots+{ }_{n} \delta_{m}\right) Q_{m}\right] \\
& \cdot \tau^{\left.\left({ }_{1} \delta_{1}+\cdots+n \delta_{1}\right) r_{1}+\cdots+{ }_{1} \delta_{m}+\cdots+n \delta_{m}\right) r_{m}}[\tau]^{*} \\
= & \exp \left[h_{1} Q_{1}(\tau)+\cdots+h_{m} Q_{m}(\tau)\right] \tau^{h_{1} r_{1}+\cdots+h_{m} r_{m}}[\tau]^{*} .
\end{aligned}
$$

Since ${ }_{j} a_{i_{1}}, \cdots, i_{n}(\tau)=[\tau]_{0}$, in view of (2.24) it is concluded that

$$
{ }_{2} \Gamma_{i}^{h_{1}, \cdots, h_{m}}(\tau)=\sum_{i_{1}+\cdots+i_{n}=2} \sum_{\gamma_{1}+\cdots+\gamma_{n}=2} \sum_{{ }_{1} \delta_{1}, \cdots,{ }_{n} \delta_{m}}^{(3)} F_{i_{1}, \cdots, i_{n} ; \gamma_{1}, \cdots, \gamma_{n} i_{1} \delta_{1}, \cdots, n}^{h_{1}, \cdots, h_{m}}
$$

where $\gamma_{1}=i_{1}, \cdots, \gamma_{n}=i_{n}$ and

(3.4a) $\quad F_{i_{1} ;}^{h_{1}}, \ldots ; \ldots, \cdots=$ generic form of the last member of $(3.3 \mathrm{c})$. 
The combined summation represented by the three displayed summation symbols involved in the second member of (3.4) is not with respect to $\left(h_{1}, \cdots, h_{m}\right)$. Hence, in view of (3.4a),

$$
\begin{gathered}
{ }_{2} \Gamma_{j}^{h_{1}, \cdots, h_{m}}(\tau)=\exp \left[h_{1} Q_{1}(\tau)+\cdots+h_{m} Q_{m}(\tau)\right] \tau^{h_{1} r_{1}+\cdots+h_{m} r_{m}}[\tau] *(\tau \text { in } R), \\
h_{1}+\cdots+h_{m}=2, \quad h_{1} \geqq 0, \cdots, h_{m} \geqq 0 ; j=1, \cdots, n
\end{gathered}
$$

If we make use of (3.5) and (3.1a), we infer that the integrand displayed in (2.30a) (for $B=2)$ is of the form

$$
\exp [Q(\tau)] \tau^{r}[\tau]^{*},
$$

where

$$
\begin{aligned}
Q(\tau) & =h_{1} Q_{1}(\tau)+\cdots+h_{m} Q_{m}(\tau)-Q_{\lambda}(\tau) ; \\
r & =h_{1} r_{1}+\cdots+h_{m} r_{m}-r_{\lambda}+\omega+p .
\end{aligned}
$$

In view of our present purposes it will be essential to integrate expressions of the form (3.6) $\left(Q(\tau)\right.$ a polynomial in $\tau^{1 / k} ; k$ an integer $\left.\geqq 1\right)$ in such a manner that the integral is of the same generic form as the integrand, with $r$ possibly changed. This can certainly be achieved by the methods of Trjitzinsky ${ }^{\text {if }} R Q(\tau)$ does not change sign in $R$. Now, when one examines the particular integrand (3.6) for which (3.6a) holds, the following is noted regarding $Q(\tau)$. If $\lambda>m$ we pick out an $h_{\alpha}(>0) \ddagger$ and write

$$
\begin{aligned}
Q(\tau)= & {\left[h_{1} Q_{1}(\tau)+\cdots+\left(h_{\alpha}-1\right) Q_{\alpha}(\tau)+\cdots+h_{m} Q_{m}(\tau)\right] } \\
& +Q_{\alpha}(\tau)-Q_{\lambda}(\tau) .
\end{aligned}
$$

Here $h_{1} \geqq 0, \cdots, h_{\alpha}-1 \geqq 0, \cdots, h_{m} \geqq 0$; thus, since one may write in consequence of $(2.9 \mathrm{a})$ and $(2.9 \mathrm{~b})$

$$
R Q_{1}(\tau) \leqq \cdots \leqq R Q_{m}(\tau)<0 \quad \quad(\tau \text { in } R),
$$

it is inferred that the real part of the expression within the brackets of the second member of (3.7) is certainly negative in $R$. In this connection we make use of the fact that

$$
h_{1}+\cdots+h_{\alpha-1}+\left(h_{\alpha}-1\right)+h_{\alpha}+\cdots+h_{m}=1,
$$

as can be seen from (3.5a). On the other hand, by (2.9c)

$$
R\left(Q_{\alpha}(\tau)-Q_{\lambda}(\tau)\right) \leqq 0 \quad(\tau \text { in } R) .
$$

Whence it is concluded that, for $\lambda>m$,

$\dagger$ Cf. $\left(T_{2}\right)$.

$\ddagger$ This is possible in view of (3.5a). 


$$
R Q(\tau)<0
$$

Moreover, by (2.9a), (2.9b), exp $[Q(\tau)] \sim 0$ in $R$. Suppose now that $\lambda \leqq m$. If $h_{\lambda}>0$ we have

$$
Q(\tau)=h_{1} Q_{1}(\tau)+\cdots+\left(h_{\lambda}-1\right) Q_{\lambda}(\tau)+\cdots+h_{m} Q_{m}(\tau)
$$

with $h_{1} \geqq 0, \cdots, h_{\lambda-1}-1 \geqq 0, \cdots, h_{m} \geqq 0$ and $h_{1}+\cdots+\left(h_{\lambda}-1\right)+\cdots+h_{m}$ $=1$. Hence $\exp [Q(\tau)] \sim 0(\tau$ in $R)$ in this case as well. If $\lambda \leqq m$ and $h_{\lambda}=0$, the following is noted. Some $h_{\alpha}(1 \leqq \alpha<\lambda) \dagger$ may be positive; by (3.8) one then has $R\left(Q_{\alpha}(\tau)-Q_{\lambda}(\tau)\right) \leqq 0$. Since

$$
\begin{gathered}
Q(\tau)=\left[h_{1} Q_{1}(\tau)+\cdots+\left(h_{\alpha}-1\right) Q_{\alpha}(\tau)+\cdots+h_{m} Q_{m}(\tau)\right]+Q_{\alpha}(\tau)-Q_{\lambda}(\tau) \\
\left(h_{1} \geqq 0, \cdots, h_{\alpha}-1 \geqq 0, \cdots, h_{m} \geqq 0 ; h_{1}+\cdots+\left(h_{\alpha}-1\right)+\cdots+h_{m}=1\right),
\end{gathered}
$$

it is inferred that, for the case under consideration, $R Q(\tau)<0$ and $\exp [Q(\tau)]$ $\sim 0(\tau$ in $R)$. In the remaining case, when $\lambda \leqq m$ and $h_{\lambda}=0$ one has $h_{1}=h_{2}=\ldots$ $=h_{\lambda}=0$. This necessitates that some $h_{\alpha}(\lambda<\alpha \leqq m) \ddagger$ should be positive, and we have

$$
\begin{aligned}
Q(\tau)=-Q_{\lambda}(\tau)+h_{\lambda+1} Q_{\lambda+1}(\tau)+\cdots+h_{m} Q_{m}(\tau) \\
\left(h_{\lambda+1} \geqq 0, \cdots, h_{m} \geqq 0 ; h_{\lambda+1}+\cdots+h_{m}=2\right) .
\end{aligned}
$$

Now $R Q(\tau)$, where $Q(\tau)$ is given by (3.9), will not necessarily maintain its sign§ in $R$, unless $\lambda=m$, in which case some $h_{\alpha}(1 \leqq \alpha<\lambda)$ would have to be positive. If, more generally, functions are considered of the form

$$
Q(\tau)=g_{1} Q_{1}(\tau)+g_{2} Q_{2}(\tau)+\cdots+g_{m} Q_{m}(\tau)=\sum_{\nu=0}^{l-1} q_{\nu} \tau^{(l-\nu) / k},
$$

where the $g_{i}(i=1, \cdots, n)$ are real, and where, unless $Q(\tau) \equiv 0$, the leading term actually present is

$$
q_{\nu_{1}} \tau^{\left(l-\nu_{1}\right) / k} \quad\left(0 \leqq \nu_{1} \leqq l-1 ; q_{\nu_{1}} \neq 0\right),
$$

it is observed that, with

$$
q_{0}{ }^{1} \tau^{l_{1} / k_{1}}, q_{0}{ }^{2} \tau^{l_{2} / k_{2}}, \cdots, q_{0}{ }^{m} \tau^{l_{m} / k_{m}}
$$

respectively denoting the leading terms in $Q_{1}(\tau), Q_{2}(\tau), \cdots, Q_{m}(\tau)$, we necessarily have

$$
\frac{l-\nu_{1}}{k} \leqq l^{\prime}
$$

$\dagger$ This may arise only if $\lambda>1$.

$\ddagger$ This may occur only if $\lambda<m$.

$\S$ Examples can be given when $R Q(\tau)$ changes sign in $R$. 
where $l^{\prime}$ is the greater one of the numbers $l_{i} / k_{i}(i=1, \cdots, m)$. The regular curves along which $R Q(\tau)=0$ will possess at infinity limiting directions $\bar{\tau}_{0}$ satisfying the equation

$$
\begin{array}{r}
R\left(q_{\nu} \tau^{\left(l-\nu_{1}\right) / k}\right)=\left|q_{\nu_{1}}\right||\tau|^{\left(l-\nu_{1}\right) / k} \cos \left[\bar{q}_{\nu_{1}}+\left(\frac{l-\nu_{1}}{k}\right) \bar{\tau}_{0}\right]=0 \\
\left(\bar{q}_{\nu_{1}}=\angle q_{\nu_{1}} ; \bar{\tau}=\angle \tau\right) .
\end{array}
$$

These directions have accordingly the values

$$
\bar{\tau}_{0}=\bar{\tau}_{0}^{(m)}=\frac{k}{l-\nu_{1}}\left[\frac{\pi}{2}+m \pi-\bar{q}_{\nu_{1}}\right] \quad(m=0, \pm 1, \pm 2, \cdots) .
$$

Thus they differ by non-zero integral multiples of $k \pi /\left(l-\nu_{1}\right)$. By (3.10c)

$$
\frac{k \pi}{l-\nu_{1}} \geqq \frac{\pi}{l^{\prime}}
$$

At this stage it will be convenient to introduce a definition.

Definition 6. Let $R$ denote a region satisfying conditions (i), (ii) of $\$ 2$. Let $Q(\tau)$ be a polynomial of the form (3.10). A region $W$ will be said to be of the order $\nu(\geqq 1)$ with respect to $Q(\tau)$ if the following conditions are satisfied.

(I) $W$ is coincident with or is a regular (cf. Definition $3, \S 2$ ) subregion of $R$.

(II) $W=W_{1}+W_{2}+\cdots+W_{\nu}$, where $W_{1}, \cdots, W_{\nu}$ are regular non-overlapping regions such that along $W_{i, i+1}$, the common boundary of $W_{i}$ and $W_{i+1}$ $(i=1, \cdots, \nu-1), R Q(\tau)=0$, while interior to $W_{i}(i=1, \cdots, \nu) R Q(\tau)$ does not change sign.

When $\nu=1$ Condition (II) is to be replaced by a statement to the effect that $R Q(\tau)$ does not change sign in $W$.

With $\nu>1$ and $\theta_{i, i+1}$ denoting the limiting direction of $W_{i, i+1}(i=1, \cdots$, $\nu-1)$ at infinity, it may be supposed without any loss of generality that

$$
\theta_{1,2}<\theta_{2,3}<\cdots<\theta_{\nu-1, \nu}
$$

One may then write

$$
\theta_{i, i+1}=\theta_{1,2}+(i-1) \frac{k \pi}{l-\nu_{1}} \quad(i=1,2, \cdots, \nu-1) .
$$

Lemma 2. With $R$ denoting a region satisfying (i) and (ii) of $\$ 2$, let $W$ be a regular subregion of $R$ of opening (cf. Definition 3 , \$2) less than $\pi / l^{\prime}$, where $l^{\prime}$ is the number referred to in the italicized statement in connection with $(3.10 \mathrm{~b})$, 
(3.10c). The region $W$ will then be of order $\leqq 2$ (cf. Definition 6) with respect to every $Q(\tau)$ of the form (3.10). $\dagger$

In consequence of this lemma, $R$ can be covered with a finite number of regions of order $\leqq 2$ with respect to every $Q(\tau)$ of the form (3.10). The inequality " $\leqq 2$ " cannot be replaced by the equality " $=1$ " because, generally, it is impossible to find a regular subregion $W$ of $R$ (of opening however small) such that for no $Q(\tau)$ of the form (3.10) is there a curve $R Q(\tau)=0$ interior to $W$.

The truth of this lemma can be inferred as follows. Consider a region $W=R\left(\alpha_{1}, \alpha_{2}\right)$ (cf. Definition 3) for which, as required by the lemma, $0 \leqq \alpha_{2}-\alpha_{1}<\pi / l_{1}$. Unless implied otherwise we take a fixed set of numbers $g_{1}, \cdots, g_{m}$. If $Q(\tau) \equiv 0, W$ will be of order unity with respect to this $Q(\tau)$. If $Q(\tau) \not \equiv 0$ let the regular curves (Definition 2) be designated by

$$
B_{1}, B_{2}, \cdots \text {, }
$$

and let the limiting directions at infinity be the numbers

$$
\beta_{1}, \beta_{2}, \cdots,
$$

respectively. It is a matter of notation to arrange these numbers so that

$$
\beta_{1}<\beta_{2}<\cdots ; \beta_{1} \leqq \alpha_{1}
$$

and so that $B_{1}$, if not coincident with the boundary of $W$ for which limiting direction is $\alpha_{1}$, lies exterior to $W$ in such a way that between $B_{1}$ and the mentioned boundary of $W$ there is no curve $B_{i}(i=2,3, \cdots)$ for which

$$
\beta_{1}<\beta_{i} \leqq \alpha_{1} .
$$

The implication of the above is that, if (3.11a) holds for $i=2$, the curve $B_{2}$ must extend interior to $W$ (in which case necessarily $\beta_{2}=\alpha_{1}$ ).

For some subscript $\nu(>1) B_{\nu}$ will be either coincident with the boundary of $W$, for which the limiting direction is $\alpha_{2}$, or it will lie exterior to $W$ so that between the boundary of $W$, just referred to, and $B_{\nu}$ there is no curve $B_{i}$ $(i<\nu)$ such that

$$
\alpha_{2} \leqq \beta_{i}<\beta_{\nu} .
$$

If, however, (3.11b) holds for $i=\nu-1$, the above necessitates that we should have $\alpha_{2}=\beta_{\nu-1}$, while $B_{\nu-1}$ extends interior to $W$.

Corresponding to a particular $Q(\tau)(\not \equiv 0)$ of the form (3.10), $W$ has been covered by a succession of $\nu-1$ adjacent regions separated by the curves $B_{2}, B_{3}, \cdots, B_{\nu-1}$, all interior to $W$. Designate these regions by 


$$
R^{1,2}, R^{2,3}, \cdots, R^{\nu-1, \nu} \quad\left(R^{i, i+1}=R\left(\beta_{i}, \beta_{i+1}\right)\right) .
$$

With the leading term in $Q(\tau)$ given by (3.10a) one has, in view of the statements preceding Definition 6,

$$
\beta_{\nu-1}-\beta_{2}=(\nu-3) \frac{k \pi}{l-\nu_{1}} \geqq(\nu-3) \frac{\pi}{l^{\prime}},
$$

whenever $\nu>3$.

When $\nu \leqq 3$ there are at most two regions (3.12), and $W$ will be of order $\leqq 2$. If $\nu>3$ consider the region

$$
R^{2,3}+\cdots+R^{\nu-2, \nu-1}=R\left(\beta_{2}, \beta_{\nu-1}\right) .
$$

It will be interior to $W . \dagger$ Now $W=R\left(\alpha_{1}, \alpha_{2}\right)$. Thus

$$
\beta_{v-1}-\beta_{2} \leqq \alpha_{2}-\alpha_{1}<\frac{\pi}{l_{1}} \text {. }
$$

This, in view of (3.12a) implies that

$$
(\nu-3) \frac{\pi}{l^{\prime}}<\frac{\pi}{l^{\prime}}, \quad \nu>3 \quad \text { ( } \nu \text { an integer). }
$$

These inequalities are, however, incompatible. Hence $\nu \leqq 3$ necessarily and $W$ is at most of the second order.

In the sequel, unless stated otherwise, $W$ is to denote a particular regular subregion of $R$ with opening $<\pi / l^{\prime}$.

4. Integrations. Let $W=R\left(\alpha_{1}, \alpha_{2}\right), \alpha_{1}<\alpha_{2}$ (cf. Definition 3, §2) be a region such that $\alpha_{2}-\alpha_{1}<\pi / l^{\prime}$. Consider the problem

$$
y^{(1)}(t)=e^{Q(t)} t^{r} a(t)
$$

where, unless $Q(t) \equiv 0$,

$$
Q(t)=\sum_{\nu=\nu_{1}}^{l-1} q_{\nu} t^{(l-\nu) / k} \quad\left(0 \leqq \nu_{1} \leqq l-1 ; q_{\nu_{1}} \neq 0\right)
$$

and $\left(l-\nu_{1}\right) / k \leqq l^{\prime}$. Assume $a(t)$ to be of the form

$$
a(t)=[t]^{*}
$$

(cf. Definition 4, §2).

It is not difficult to see that Lemma 2 holds when $Q(t)$, instead of being assumed to be of the form (3.10) is given by (4.1a). The essential fact is that the inequality subsequent to (4.1a) holds. Thus $W$ is of order $\leqq 2$ (with respect to all the $Q(t)$ of stated type-provided, of course, that $l^{\prime}$ is fixed). In this

$\dagger$ That is, all of its points, with the possible exception of those on the circular part of its boundary, are interior to $W$. 
connection "order" is specified according to Definition $6, \S 3$. When $Q(t) \not \equiv 0$ we have $W=W_{1}+W_{2}$, where

$$
W_{1}=R\left(\alpha_{1}, \beta_{2}\right), \quad W_{2}=R\left(\beta_{2}, \alpha_{2}\right) \quad\left(\alpha_{1} \leqq \beta_{2} \leqq \alpha_{2}\right),
$$

unless

$$
W=W_{1}=R\left(\alpha_{1}, \alpha_{2}\right) .
$$

The symbols involved here have the same significance as in $\$ 3$. With $B_{i}$ denoting the regular curves satisfying the equation $R Q(t)=0$ and $\beta_{i}$ denoting the limiting direction of $B_{i}$ we have $B_{2}$ interior to $W$ (in case (4.2)) or there are no curves $B_{i}$ interior to $W$. The curve $B_{1}$ is exterior to $W$ or is coincident with the right boundary $\dagger$ of $W$ and the curve $B_{3}$ is exterior to $W$ or is coincident with the left boundary of $W$. However, in view of our present purposes, it will be necessary to examine this case in greater detail, taking advantage of the conditions satisfied by $W$.

When $W=W_{1}$ the integration methods given in $\left(\mathrm{T}_{2}\right)$ are applicable. In the case of $W=W_{1}+W_{2}$ an extension of these methods will be necessary. The latter case will now be considered.

By hypothesis $a(t) \sim \alpha(t)(t$ in $W)$, where

$$
\alpha(t)=\{t\}^{*}
$$

(cf. Definition 1, §2). As demonstrated in $\left(\mathrm{T}_{2}\right)$ the formal equation

$$
s^{(1)}(t)=e^{Q(t)} t^{r} \alpha(t),
$$

associated with (4.1), possesses a formal solution

$$
s(t)=e^{Q(t)} t^{r+w} \sigma(t),
$$

where

$$
\sigma(t)=\{t\}^{*}, \quad w=1-\left(\frac{l-\nu_{1}}{k}\right) \quad\left(1-l^{\prime} \leqq w<1\right) .
$$

Retaining in the power series involved in $\sigma(t)$ the first $\beta_{1}$ terms only, we obtain a certain function $\sigma_{\beta_{1}}(t)$. Apply now the transformation

$$
y(t)=e^{Q(t)} t^{r+w} \sigma_{\beta_{1}}(t)+z(t)
$$

to the given equation (4.1). It is observed that $z(t)$ will satisfy

$$
z^{(1)}(t)=\zeta(t)
$$

where

† The "right" boundary of $W$ is that one of the two regular curves, forming part of the boundary of $W$, which appears on the right when the region $W$ is faced from the origin. 


$$
\begin{aligned}
\zeta(t) & =e^{Q(t)} t^{r} a(t)-\frac{d}{d t}\left[e^{Q(t)} t^{r+w} \sigma_{\beta_{1}}(t)\right] \\
& =e^{Q(t)} t^{-\beta} b(t) \quad(|b(t)| \leqq b ; t \text { in } W),
\end{aligned}
$$

as can be seen from the developments given in $\left(\mathrm{T}_{2}\right)$. The essential fact to be noted is that in (4.6a) $\beta\left(=\beta\left(\beta_{1}\right)\right)$ can be made as large as desired by choosing $\beta_{1}$ sufficiently great. The function $b(t)$ will, of course, be analytic in $W(t \neq \infty)$.

Suppose, for instance, that $R Q(t)>0$ interior to $W_{1}$. Let $t_{0}$ denote the point where $B_{2}$ meets the circular part of the boundary of $W$. A solution of (4.6) will be defined as follows:

$$
\begin{aligned}
z(t) & =\zeta_{0}+\int_{t_{0}}^{t} \zeta(\tau) d \tau \\
\zeta_{0} & =\int_{\infty}^{t_{0}} \zeta(\tau) d \tau .
\end{aligned}
$$

In (4.7) the path of integration is interior to $W_{2}$ when $t$ is interior to $W_{2}$,and it is interior to $W_{1}$ when $t$ is interior to $W_{1}$. This path is along $B_{2}$ when $t$ is on $B_{2}$. In (4.7a) the path is along $B_{2}$. We deliberately avoid using the notation

$$
z(t)=\int_{\infty}^{t} \zeta(\tau) d \tau
$$

unless further qualifications are introduced. This is because, for $t$ in $W_{1}$, there may exist paths $(t, \infty)$ extending interior to $W_{1}$ for which the displayed integral (4.7b) diverges. Formulas (4.7) and (4.7a) define a solution of (4.6) analytic throughout $W(t \neq \infty)$.

If $t$ is in $W_{1}$ and there are no curves $\dagger$

$$
\frac{\partial R Q(t)}{\partial \theta}=0 \quad\left(t=|t| \exp \left[(-1)^{1 / 2} \theta\right]\right) \ddagger
$$

between $t$ and $B_{2}$, we shall write

$$
z(t)=\left(\int_{\infty}^{t^{\prime}}+\int_{t^{\prime}}^{t}\right) \zeta(\tau) d \tau \quad\left(\left|t^{\prime}\right|=|t|\right) .
$$

The first integration displayed here is along $B_{2}$; the second is along an arc of the circle $|\tau|=|t|$. With the aid of (4.6a) it follows that

$\dagger$ The whole discussion here and in the sequel is given sufficiently far away from the origin so that no two distinct regular curves, under consideration, intersect.

$\ddagger$ All such regular curves have limiting directions distinct from those of the regular curves $R Q(t)=0$ (cf. $\left.\left(\mathrm{T}_{2}\right)\right)$. 


$$
|z(t)| \leqq b\left(\int_{\infty}^{t^{\prime}}+\int_{t^{\prime}}^{t}\right) e^{R Q(\tau)}|\tau|^{-\beta+2}\left|\frac{d \tau}{\tau^{2}}\right| .
$$

By taking $\beta_{1}$ sufficiently great the inequality $-\beta+2 \geqq 0$ will be secured. Since $R Q(\tau)$ increases monotonically as $\tau$ describes the circular arc $|\tau|=|t|$ from $t^{\prime}$ to $t$, it is inferred that

$$
e^{R Q(\tau)}|\tau|^{-\beta+2}
$$

attains its upper bound, for the combined path under consideration, at $t$. Thus

$$
|z(t)| \leqq b\left|e^{Q(t)} t^{-\beta+2}\right|\left(\int_{\infty}^{t^{\prime}}+\int_{t^{\prime}}^{t}\right)\left|\frac{d \tau}{\tau^{2}}\right| .
$$

As established in $\left(\mathrm{T}_{2}\right)$ we have, when $\tau=r \exp \left[(-1)^{1 / 2} \theta\right]$, is on $B_{2}$,

$$
\theta-\beta_{2}=f_{0} r^{-p / k}+f_{1} r^{-(p+1) / k}+\cdots \quad(p \text { an integer } \geqq 1),
$$

the series here involved being convergent for $r \geqq r_{1}$ ( $r_{1}$ sufficiently great). Hence along $B_{2}$

$$
|d \tau|=\left[r^{2}(d \theta)^{2}+\left(d r^{2}\right)\right]^{1 / 2}=\left(1+g_{1} r^{-1 / k}+g_{2} r^{-2 / k}+\cdots\right)|d r|,
$$

so that along $B_{2}$

$$
|d \tau|<g|d r|
$$

where $g(>0)$ is independent of $\tau$ and depends only on the curve $B_{2}$. Thus

$$
\int_{\infty}^{t^{\prime}}\left|\frac{d \tau}{\tau^{2}}\right|<\frac{g}{|t|} \quad\left(\left|t^{\prime}\right|=|t|\right) .
$$

On the other hand,

$$
\int_{t^{\prime}}^{t}\left|\frac{d \tau}{\tau^{2}}\right|=\int_{t^{\prime}}^{t}\left|\frac{d \theta}{\tau}\right|=\frac{1}{|t|}\left(\angle t^{\prime}-\angle t\right)<\frac{g^{\prime}}{|t|},
$$

since $\angle t^{\prime}-\angle t$ is obviously bounded when $t^{\prime}$ is on $B_{2}$ and $t\left(|t|=\left|t^{\prime}\right|\right)$ is any other point in $W$. By (4.10), (4.10a) it is inferred from (4.9a) that

$$
|z(t)|<\left(g+g^{\prime}\right) b\left|e^{Q(t)} t^{-\beta+1}\right|
$$

when $t$ is in $W_{1}$ while no regular curves (4.8) are between $t$ and $B_{2}$. In other words, if no curve (4.8) extends interior to $W_{1}$ the inequality (4.11) will certainly. hold throughout $W_{1}$.

Suppose now there are regular curves (4.8) interior to $W_{1}$. As indicated in $\left(\mathrm{T}_{2}\right)$ there is just one curve (4.8) between $B_{1}$ and $B_{2}$. Hence there will be only 
one curve (4.8) interior to $W_{1}$. Let $\lambda_{0}$ be its limiting direction. Then $\dagger$

$$
\alpha_{1} \leqq \lambda_{0}<\beta_{2}
$$

and

$$
\lambda_{0}=\frac{1}{2}\left(\beta_{1}+\beta_{2}\right) .
$$

We need to obtain an inequality for $|z(t)|$ when $t$ is in $W_{1}$ between the curve (4.8) (with the limiting direction $\lambda_{0}$ ) and the right boundary of $W_{1}$ (i.e., of $W$ ). (4.7), (4.7a) will now be written in the form

$$
z(t)=\zeta_{0}+\zeta_{1}+\left(\int_{t_{0}^{\prime}}^{t^{\prime}}+\int_{t^{\prime}}^{t}\right) \zeta(\tau) d \tau \quad\left(\left|t^{\prime}\right|=|t|\right)
$$

Here

$$
\zeta_{1}=\int_{t_{0}}^{t_{0}^{\prime}} \zeta(\tau) d \tau
$$

with the integration extended over the circular part of the boundary of $W_{1}$ ( $t_{0}$ and $t_{0}^{\prime}$ representing the points where $B_{2}$ and the right boundary of $W$ meet the circular arc). The first integral displayed in (4.13) is along the right boundary of $W$. The second is along an arc of the circle $|\tau|=|t|$. If $B_{1}$ had the same limiting direction at infinity as the right boundary of $W$, that is, if $\beta_{1}=\alpha_{1}$, a contradiction would necessarily follow, since it has been previously assumed that $\alpha_{1}<\beta_{2} \leqq \alpha_{2}$. In fact, one would have $\beta_{2}-\beta_{1}\left(=\beta_{2}-\alpha_{1}\right) \leqq \alpha_{2}-\alpha_{1}$ $<\pi / l^{\prime} ;$ on the other hand, $\ddagger$

$$
\beta_{2}-\beta_{1}=\frac{k \pi}{l-\nu_{1}} \geqq \frac{\pi}{l^{\prime}} .
$$

Hence, whenever there exists a curve $R Q(t)=0$ interior to $W, \alpha_{1}>\beta_{1}$. We will have necessarily

$$
\begin{array}{r}
a=\cos \left(\bar{q}_{\nu_{1}}+\frac{l-\nu_{1}}{k} \alpha_{1}\right)>0 . \\
\quad R Q(t)=\left|q_{\nu_{1}}\right||t|^{\left(l-\nu_{1}\right) / k} \cos \left(\bar{q}_{\nu_{1}}+\frac{l-\nu_{1}}{k} \theta\right)+\cdots
\end{array}
$$

and

$$
\frac{\partial}{\partial \theta} R Q(t)=-\left|q_{\nu_{1}}\right||t|\left(l-\nu_{1}\right) / k\left(\frac{l-\nu_{1}}{k}\right) \sin \left(\bar{q}_{\nu_{1}}+\frac{l-\nu_{1}}{k} \theta\right)+\cdots .
$$

Thus the limiting directions of the curves $R Q(t)=0$ are the values of $\theta$ for which $\cos \left(\bar{q}_{\nu_{1}}+\left(\left(l-\nu_{1}\right) / k\right) \theta\right)$ $=0$, while those of the curves $(4.8)$ are the values of $\theta$ for which $\sin \left(\bar{q}_{\nu_{1}}+\left(\left(l-\nu_{1}\right) / k\right) \theta\right)=0$.

$\ddagger$ This is a consequence of an inequality stated in connection with (4.1a). 
Accordingly along the right boundary $\dagger$ of $W$

$$
R Q(\tau)=a\left|q_{\nu_{1}}\right||\tau|^{\left(l-\nu_{1}\right) / k}\left(1+g_{t}\right) \quad\left(a\left|q_{\nu_{1}}\right|>0\right),
$$

where $g_{\tau} \rightarrow 0$ as $|\tau| \rightarrow \infty$. In consequence of (4.14) it is inferred that there exists a number $r(\beta)$ so that, for all $t^{\prime}$ on the right boundary of $W$ and such that $\left|t^{\prime}\right| \geqq r(\beta)$, we have

$$
\left|e^{Q(\tau)} \tau^{-\beta}\right| \leqq\left|e^{Q\left(t^{\prime}\right)}\left(t^{\prime}\right)^{-\beta}\right|
$$

when $\tau$ is restricted to the part of the mentioned boundary between $t_{0}^{\prime}$ and $t^{\prime}$. Whence, in view of (4.6a),

$$
\begin{array}{rlrl}
\left|\int_{t_{0}^{\prime}}^{t^{\prime}} \zeta(\tau) d \tau\right| & \leqq b \int_{t_{0}^{\prime}}^{t^{\prime}}\left|e^{Q(\tau)} \tau^{-\beta}\right||d \tau| \leqq b\left|e^{Q\left(t^{\prime}\right)}\left(t^{\prime}\right)^{-\beta}\right| \int_{t_{0}^{\prime}}^{t^{\prime}}|d \tau| \\
& <b_{1}\left|e^{Q\left(t^{\prime}\right)}\left(t^{\prime}\right)^{-\beta+1}\right| & \left(\left|t^{\prime}\right| \geqq r(\beta)\right) .
\end{array}
$$

Take $b^{\prime}\left(\geqq b_{1}\right)$, depending on $\beta$, sufficiently great so that

We then have

$$
\left|\int_{t_{0}^{\prime}}^{t^{\prime}}\right|<b^{\prime}\left|e^{Q\left(t^{\prime}\right)}\left(t^{\prime}\right)^{-\beta+1}\right| \quad\left(\left|t^{\prime}\right|<r(\beta)\right) .
$$

$$
\left|\int_{t_{0}^{\prime}}^{t^{\prime}}\right|<b^{\prime}\left|e^{Q\left(t^{\prime}\right)}\left(t^{\prime}\right)^{-\beta+1}\right|
$$

for all $t^{\prime}$ on the right boundary of $W$.

On the circular arc $\left(t^{\prime}, t\right)$ constituting the path of integration in the second integral displayed in (4.13), $R Q(\tau)$ attains its upper bound at $t$ ( $t$ between the right boundary of $W_{1}$ and the curve (4.8) extending interior to $W_{1}$ ). Thus by the same reasoning as before one obtains

$$
\left|\int_{t^{\prime}}^{t} \zeta(\tau) d \tau\right| \leqq b g^{\prime}\left|e^{Q(t)} t^{-\beta+1}\right|
$$

If we apply the relations

$$
\left|t^{\prime}\right|=|t|, \quad\left|e^{Q\left(t^{\prime}\right)}\right| \leqq\left|e^{Q(t)}\right|
$$

to $(4.15 \mathrm{a})$, in consequence of (4.16) we infer that

$$
\left|\left(\int_{t_{0}^{\prime}}^{t^{\prime}}+\int_{t^{\prime}}^{t}\right) \zeta(\tau) d \tau\right|<b^{\prime \prime}\left|e^{Q(t)} t^{-\beta+1}\right|,
$$

where $b^{\prime \prime}$ may depend on $\beta$. When $t$ is between the right boundary of $W$ and the curve (4.8) (extending in $W_{1}$ ), we have

$\dagger$ Use is made of the fact that, if $\theta=f(r)\left(\tau=r \exp \left[(-1)^{1 / 2} \theta\right)\right]$ is the equation of this boundary, one has $\lim f(r)=\alpha_{1}$ as $r \rightarrow \infty$. 


$$
\left|e^{-Q(t)} t^{\beta+1}\right| \leqq\left|e^{-Q\left(t^{\prime}\right)}\left(t^{\prime}\right)^{\beta+1}\right| \leqq b_{1}^{\prime \prime}
$$$$
\left(\left|t^{\prime}\right|=|t|\right),
$$

where $t^{\prime}$ has the significance indicated before and $b_{1}^{\prime \prime}$ may depend on $\beta$. With the aid of (4.17) and (4.17a) from (4.13) we obtain the inequality

$$
|z(t)|<b_{2}\left|e^{Q(t)} t^{-\beta+1}\right|
$$

$\left(b_{2}\right.$ dependent on $\left.\beta\right)$

for $t$ in the region specified in connection with (4.17a). In view of (4.11) it is seen that (4.18) is valid throughout $W_{1}$ in any case.

When $t$ is in $W_{2}$ there are the following four possibilities.

Case 1. The regular curve (4.8) whose limiting direction is $\left(\beta_{1}+\beta_{2}\right) / 2$ is coincident with the left boundary of $W_{2}$ or extends interior to $W_{2}$.

Case 2. The curve (4.8) mentioned in Case 1 extends exterior to $W_{2}$, while $\alpha_{2}>\beta_{2}$.

Case 3. $\alpha_{2}=\beta_{2}$ and there exists a regular curve

$$
R Q(\tau)=-h(<0), \dagger
$$

with limiting direction $\beta_{2}$, which is coincident with the left boundary of $W_{2}$ or extends exterior to $W_{2}$.

Case 4. $\alpha_{2}=\beta_{2}$ and for no $h(>0)$ is there a curve (4.19) satisfying the conditions laid down in the formulation of Case 3.

In Case 1 one has

$$
R Q(\tau)=-a^{\prime}|\tau|^{(l-\nu) / k}\left(1+g_{r}\right)
$$

where $g_{\tau} \rightarrow 0$ as $|\tau| \rightarrow \infty$ along the curve (4.8) under consideration. The function $z(t)$, as defined by (4.7), can be expressed as follows:

$$
z(t)=\left(\int_{\infty}^{t_{1}}+\int_{t_{1}}^{t}\right) \zeta(\tau) d \tau \quad\left(\left|t_{1}\right|=|t|\right) .
$$

Here the first displayed integration is along the curve (4.8); the second is along an arc of the circle $|\tau|=|t|$. With the radius of the circular part of the boundary of $W$ sufficiently great (but independent of $\beta$ ), the upper bound of

$$
\left|e^{Q(\tau)} \tau^{-\beta+2}\right|
$$

is attained at $t_{1}$ when $\tau$ is the variable of integration in the first displayed integral of (4.21). This follows from (4.20). On the other hand, along the arc $\left(t_{1}, t\right),|\exp [Q(\tau)]|$ increases monotonically, attaining the upper bound at $t$. Thus an inequality like (4.9b) is obtained. With the aid of a reasoning closely analogous to that employed in deriving (4.11) it is inferred that in Case 1

$$
|z(t)|<a_{1}\left|e^{Q(t)} t^{-\beta+1}\right|
$$

$\left(t\right.$ in $\left.W_{2}\right)$.

$\dagger$ Regarding such curves cf. $\left(\mathrm{T}_{2}\right)$. 
In Case 2, a relation like (4.20) will hold along the left boundary of $W_{2}$. This follows from the inequality $\beta_{3}>\alpha_{2}>\beta_{2}$. We express $z(t)$ as in (4.21) with the first integration involved there along the left boundary of $W_{2}$. Making an assumption as stated in italics subsequent to (4.21) we conclude, as in Case 1, that (4.21a) holds (for $t$ in $W_{2}$ ) in Case 2 as well.

In Case $3,-h \leqq R Q(\tau) \leqq 0$ for $\tau$ in $W_{2}$. Thus

$$
e^{-h} \leqq\left|e^{Q(\tau)}\right| \leqq 1
$$

$$
\left(\tau \text { in } W_{2}\right) \text {. }
$$

On writing (4.21) with the path of integration as in Case 2, we note that

$$
\left|e^{Q(\tau)} \tau^{-\beta+2}\right| \leqq|t|^{-\beta+2}
$$

when $\tau$ is on the combined path of integration. In view of (4.21) one accordingly has (cf. (4.6a))

$$
|z(t)| \leqq b|t|^{-\beta+2}\left(\int_{\infty}^{t_{1}}+\int_{t_{1}}^{t}\right)\left|\frac{d \tau}{\tau^{2}}\right| .
$$

Inequalities (4.10), (4.10a) will hold with $g$ and $g^{\prime}$ having a meaning analogous to that previously assigned. Whence it is concluded that

$$
|z(t)|<\left(g+g^{\prime}\right) b|t|^{-\beta+1} \quad\left(t \text { in } W_{2}\right) .
$$

In view of (4.22) it is inferred that (4.21a) holds in Case 3, provided that we take

$$
a_{1} \geqq e^{h}\left(g+g^{\prime}\right) b .
$$

In the remaining Case $4, R Q(\tau)$ will be monotonically decreasing along the left boundary of $W_{2}$ (as $|\tau| \rightarrow \infty$ along this boundary), provided that the radius of the circular part of the boundary is taken sufficiently great. Along any circular arc

$$
\left(t_{1}, t\right) \quad\left(\left|t_{1}\right|=|t|\right),
$$

where $t_{1}$ is on the left boundary of $W_{2}$ and $t$ is in $W_{2}, R Q(\tau)$ will attain its upper bound at $t$. The repetition, then, of the argument of Case 2 will yield an analogous inequality for $|z(t)|$.

In view of the statement previously made in connection with (4.18) it is now seen that (4.18) (with a suitable $b_{2}$, dependent on $\beta$ ) will hold throughout $W=W_{1}+W_{2}$. If we write

$$
z(t)=e^{Q(t)} t^{r+w_{Z_{1}}(t)}
$$

it is accordingly observed that

$$
\left|z_{1}(t)\right|<b_{2}{ }^{\prime}|t|^{-\beta^{\prime}} \quad\left(\beta^{\prime}=\beta-1+r^{\prime}+w\right) \dagger
$$

$\dagger r^{\prime}=$ real part of $r$. 
for $t$ in $W_{1}+W_{2}$. Moreover, $\beta^{\prime} \rightarrow \infty$ with $\beta_{1} \rightarrow \infty$. By virtue of (4.24) and (4.5) it is concluded that the following is true for every positive integer $\alpha$.

The problem formulated at the beginning of this section possesses a solution $y_{\alpha}(t)$, analytic in $W_{1}+W_{2}(t \neq \infty)$, of the form

$$
y_{\alpha}(t)=e^{Q(t)} t^{r+w} \eta_{\alpha}(t),
$$

where

$$
\eta_{\alpha}(t) \sim \sigma(t)
$$$$
\left(t \text { in } W_{1}+W_{2}\right)
$$

to $\alpha$ terms (cf. (4.4a)). Let $\alpha_{1}$ be any integer $>\alpha$. One has

$$
y_{\alpha_{1}}(t)-y_{\alpha}(t) \equiv c_{\alpha_{1}, \alpha}=e^{Q(t)} t^{r+w}\left(\eta_{\alpha_{1}}(t)-\eta_{\alpha}(t)\right) .
$$

With the aid of (4.25a) (stated for $\alpha$ and for $\alpha_{1}$ ) it is concluded that the constant $c_{\alpha_{1}, \alpha}$ must be zero. To demonstrate this it is sufficient to let $t$ recede to infinity along the curve $R Q(t)=0$, separating $W_{1}$ and $W_{2}$, and to note that the last member in $(4.25 \mathrm{~b})$ will then manifestly approach zero. Thus all the solutions $y_{\alpha}(t)$ are identical. Whence it can be asserted that (4.1) has a solution $y(t)$, analytic in $W_{1}+W_{2}(t \neq \infty)$, of the form

$$
\begin{gathered}
y(t)=e^{Q(t)} t^{r+w} \eta(t), \\
\eta(t) \sim \sigma(t)
\end{gathered}
$$$$
\left(\operatorname{tin} W_{1}+W_{2}\right)
$$

(cf. (4.4a)), the asymptotic relations being valid in the ordinary sense. The above will hold also when $R Q(t)>0$ interior to $W_{1}$ and $R Q(t)<0$ in $W_{2}$.

We shall now consider briefly the case of (4.2a). The curves $B_{1}, B_{2}$ will both be exterior to $W=W_{1}$, or one of them will be coincident with a boundary of $W_{1}$, while the other one is exterior to $W_{1}$. One now has

$$
\beta_{1} \leqq \alpha_{1}<\alpha_{2} \leqq \beta_{2} \quad\left(\alpha_{2}-\alpha_{1}<\frac{\pi}{l^{\prime}} \leqq \beta_{2}-\beta_{1}\right) .
$$

Suppose first that $R Q(t)<0$ (interior to $W_{1}$ ). If the regular curve (4.8) with the limiting direction $\left(\beta_{1}+\beta_{2}\right) / 2$ is not exterior to $W_{1}$ the function $z(t)$ (solution of (4.6)) will be expressed as an integral extending from infinity along the curve (4.8) to a point $t_{1}\left(\left|t_{1}\right|=|t|\right)$ and from $t_{1}$ to $t$ along an arc of the circle $|\tau|=|t|$. If the mentioned curve (4.8) is exterior to $W_{1}$, an inequality for $|z(t)|$ is obtained with the aid of an integration, extending from infinity to $t_{1}\left(\left|t_{1}\right|=|t|\right)$ along that one of the two regular boundaries of $W$ which is nearer to the curve (4.8); from $t_{1}$ the path of integration is continued (away from the curve (4.8) towards $t$ ) along an arc of the circle $|\tau|=|t|$. With the radius of the circular part of the boundary of $W_{1}$ sufficiently great (this choice can be made independently of $\beta, \beta_{1}$ ) it is observed that 


$$
\left|e^{Q(\tau)}\right|, \quad\left|e^{Q(\tau)} \tau^{-\beta}\right|
$$

will be monotonically increasing along the specified paths, from infinity to $t$. With the aid of the reasoning previously employed in proving (4.26), (4.26a) for $t$ in $W_{1}+W_{2}$ the truth of (4.26), (4.26a) is again made evident in the new case under consideration as well; the remarks regarding the choice of path are to be noted.

Suppose now that interior to $W_{1}, R Q(t)>0$. The following is observed. If, for instance, the right boundary of $W_{1}$ is considered, either

$$
\left|e^{Q(\tau)} \tau^{-\beta}\right| \rightarrow \infty
$$

(for every $\beta>0$ )

as $|\tau| \rightarrow \infty$ along this boundary, or

$$
\left|e^{Q(\tau)} \tau^{-\beta}\right| \rightarrow 0
$$

as $|\tau| \rightarrow \infty$ along the mentioned boundary. We have $\beta_{1} \leqq \alpha_{1}<\alpha_{2} \leqq \beta_{2}$. It is observed that (4.28) will hold $\dagger$ when $\alpha_{1}>\beta_{1}$; (4.28) may hold even when $\alpha_{1}=\beta_{1}$. However, with $\alpha_{1}=\beta_{1}$, the case (4.28a) will sometimes occur. Since the equalities

$$
\alpha_{1}=\beta_{1}, \quad \alpha_{2}=\beta_{2}
$$

cannot be satisfied at the same time, only one of the following two cases may present itself (when $R Q(t)>0$ interior $W=W_{1}$ ).

Case $\left(a_{1}\right)$. Along both regular boundaries of $W_{1}$ (4.28) is satisfied.

Case $\left(\mathrm{a}_{2}\right)$. Along one of the boundaries of $W_{1}$ (4.28) holds, while along the other we have $(4.28 \mathrm{a})$.

In the Case $\left(\mathrm{a}_{1}\right)$ if the curve (4.8) (between $B_{1}$ and $B_{2}$ ) is exterior to $W_{1}$ or is coincident with one of the regular boundaries of $W_{1}$, the path of integration is to be taken from a point on the circular part of the boundary of $W_{1}$ along that one of the regular boundaries of $W_{1}$ which is further removed from the mentioned curve (4.8). Along this boundary of $W_{1}$ integration is to be extended to $t_{1}\left(\left|t_{1}\right|=|t|\right)$. The path is further extended along an arc of the circle $|\tau|=|t|$ from $t_{1}$ to $t\left(t\right.$ in $\left.W_{1}\right)$. If, in the Case $\left(a_{1}\right)$, a curve (4.8) extends interior to $W_{1}$, the path is taken from $t_{0}$, the intersection of (4.8) with the circular part of the boundary of $W_{1}$, to $t$. When $t$ is, for instance, between the curve (4.8) and the right boundary of $W_{1}$, the path is deformed so as to extend from $t_{0}$ along the circular part of the boundary of $W_{1}$ to the intersection with the right boundary of $W_{1}$; the path is further continued along the latter boundary to $t_{1}\left(\left|t_{1}\right|=|t|\right)$ and from $t_{1}$ it is finally extended along an arc of the circle $|\tau|=|t|$ to $t$. An analogous deformation is made when $t$ is in $W_{1}$ to the left of the curve (4.8) under consideration. Given $\alpha$,

† Along the left boundary of $W_{1}(4.28)$ will certainly hold if $\alpha_{2}<\beta_{2}$. 
however large, a solution (4.25), (4.25a) ( $t$ in $W_{1}$ ) can be obtained. The various solutions $y_{\alpha}(t)$ will in general differ by constants distinct from zero. However, any particular one of the $y_{\alpha}(t)$ will maintain in the ordinary sense and throughout $W_{1}$ the asymptotic form of the formal solution. The latter fact is due to the relation

$$
e^{-Q(t)} \sim 0
$$

$\left(t\right.$ in $\left.W_{1}\right)$

which certainly holds in Case $\left(a_{1}\right)$. The above choice of the path having been made, the following property will hold. If $|t| \geqq r_{\beta}\left(r_{\beta}\right.$ sufficiently great), the upper bound of $\left|\tau^{-\beta} \exp [Q(\tau)]\right|$, for $\tau$ on the path under consideration, will be attained at $\tau=t$.

Consider now Case $\left(a_{2}\right)$. Suppose, for instance, that (4.28a) holds along the left boundary of $W_{1}$; let $\beta(>0)$ be sufficiently great so that we have (4.28a). In this case integration will be precisely as in the previously discussed case when $W=W_{1}+W_{2}$, where $W_{1}$ and $W_{2}$ are separated by the curve $B_{2}$ along which $R Q(t)=0, R Q(t)>0$ in $W_{1}$, and $t$ is in $W_{1}$ (cf. the developments stated in connection with (4.8)-(4.18)). One needs only to replace $B_{2}$ by the left boundary of $W_{1}$. The result will be precisely analogous to the one obtained in the previous case.

Consider now the following modified problem:

$$
\begin{aligned}
y^{(1)}(t) & =e^{Q(t)} t^{r} a(t), \\
Q(t) & =\text { polynomial in } t^{1 / k}, \\
a(t) & =[t]^{*} \\
e^{Q(t)} & \sim 0
\end{aligned}
$$$$
\left(t \text { in } R^{\prime}\right) \text {, }
$$

Here $R^{\prime}=R\left(\alpha_{1}, \alpha_{2}\right)\left(\alpha_{1}<\alpha_{2}\right)$ is a regular region (cf. Definition $\left.3, \S 2\right)$. In view of $(4.29 \mathrm{c})$ it is not difficult to infer that not more than one curve (4.8) may extend interior to $R^{\prime}$. Hence the reasoning of the type employed in the text between (4.27) and (4.28) is again applicable leading to an analogous result.

An examination of the preceding developments of this section enables us to assert the following.

Let the region $W=R\left(\alpha_{1}, \alpha_{2}\right)$ be subject to the condition

$$
0<\alpha_{1}-\alpha_{2}<\frac{\pi}{2 l^{\prime}},
$$

and let the radius $r^{\prime}$ of the circular part of the boundary of $W$ be suitably great. With $t$ in $W$ and $t_{1}\left(\left|t_{1}\right|=|t|\right)$ on a regular boundary of $W$ or on a regular curve $R Q(\tau)=0$ or on a regular curve (4.8), as the case may be, the path of integration indicated in the previous discussion extends towards $t_{1}$ along one of the mentioned curves either from infinity or from a point 
on the circular part of the boundary of $W$ (as the case may be) $\dagger \dagger$ from $t_{1}$ the path extends towards $t$ along an arc of the circle $|\tau|=|t|$. The upper bound of $\left.|\exp [Q(\tau)]| \tau\right|^{-\gamma \mid}(\gamma>0)$, when $\tau$ is on such a path, is attained at $\tau=t$ (at least for $|t| \geqq r^{\prime \prime}>r^{\prime}$ ). A similar statement (with $\gamma=0$ and $W$ replaced by $R$ ) will hold, with the condition (4.30) deleted, when the problem (4.29)$(4.29 \mathrm{c})$ is under consideration.

LEMMA 3. Consider the problem represented by (4.1), (4.1a), (4.1b) with $l^{\prime}$ fixed. A solution of this problem can be evaluated as follows:

$$
y(t)=\int^{t} e^{Q(\tau)} \tau^{r}[\tau]^{*} d \tau=e^{Q(t)} t^{r+w}[t]^{*}
$$

(cf. Definition 4, §2), where

$$
1-l^{\prime} \leqq w=1-\frac{l-\nu_{1}}{k}<1,
$$

unless $Q(t) \equiv 0$, when $w=1$. For the problem (4.29)-(4.29c), with the leading term in $Q(t)$ given by $q t^{\nu^{\prime} / k}\left(q \neq 0 ; \nu^{\prime}>0\right)$, a solution of the form (4.31) will exist for $t$ in $R^{\prime}$; in this case

$$
w=1-\frac{\nu^{\prime}}{k}<1
$$

5. Formal solutions. Let $R$ be a region of positive opening, referred to in $\S 2$ in connection with (i), (ii), (9a), and (9b). Thus, when $\tau$ is interior to $R$, we have

$$
R Q_{1}(\tau) \leqq \cdots \leqq R Q_{m}(\tau)<R Q_{m+1}(\tau) \leqq \cdots \leqq R Q_{m}(\tau)
$$

Also

$$
e^{Q_{i}(\tau)} \sim 0 \quad(i=1, \cdots, m ; \tau \text { in } R) .
$$

Let $l^{\prime}$ be the number defined in the italicized statement made in connection with (3.10b), (3.10c). Finally, let $W=R\left(\alpha_{1}, \alpha_{2}\right)\left(0<\alpha_{1}<\alpha_{2} ; \alpha_{2}-\alpha_{1}<\pi / l^{\prime}\right)$ denote a subregion of $R$.

Our purpose will now be to determine the coefficients of the series (2.12) (cf. (2.12a)), that is, the

$$
\begin{gathered}
{ }_{H} \eta_{h_{1}, \cdots, h_{m} ; j}(t) \\
\left(h_{1}, \cdots, h_{m} \geqq 0 ; h_{1}+\cdots+h_{m}={ }_{H} ; j=1, \cdots, n ; \quad \text { H }=1,2, \cdots\right) .
\end{gathered}
$$

† In a certain case the path extends from a fixed point on the circular part of the boundary of $W$, along this part to the intersection with one of the regular boundaries of $W$ and from there on along the latter boundary towards $t_{1}$; from $t_{1}$ it is continued to $t$ along a circular arc. 
For $\boldsymbol{B}=1$ the functions (5.2) are seen to be of the form (3.2). With the aid of (3.2) the relations (3.5), (3.5a) were obtained; that is, the form of the

$$
{ }_{2} \Gamma_{j}^{h_{1}, \cdots, h_{m}}(\tau)
$$

$(\tau$ in $R)$

was determined.

Before proceeding further we note that, in consequence of (3.1), (3.1a), (2.30), and (2.30a), one may write

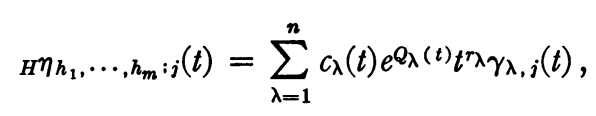

where

$$
\begin{aligned}
c_{\lambda}(t) & =\sum_{\lambda_{1}=1}^{n} \int_{H}^{t} \Gamma_{\lambda_{1}}^{h_{1}, \cdots, h_{m}}(\tau) e^{-Q_{\lambda}(\tau)} \tau^{-r_{\lambda}+\omega+p} \bar{\gamma}_{\lambda_{1}, \lambda}(\tau) d \tau, \\
\gamma_{\lambda, j}(\tau) & =[\tau]^{*}, \quad \bar{\gamma}_{\lambda_{1}, \lambda}(\tau)=[\tau]^{*} \quad(\tau \text { in } R) .
\end{aligned}
$$

Here the $\gamma_{\lambda, j}(\tau)$ and $\bar{\gamma}_{\lambda_{1}, \lambda}(\tau)$ are independent of $H, h_{1}, \cdots, h_{m}$. For $H=2$ the integrand displayed in (5.4a) has the form (3.6), (3.6a). Lemma $3, \S 4$ is seen to be applicable for evaluation of all of the integrals involved in (5.4a) for $H=2$ when $t$ is in $W$. The integral displayed in (5.4a) for $H=2$ will be of the form

$$
\exp \left[h_{1} Q_{1}(t)+\cdots+h_{m} Q_{m}(t)-Q_{\lambda}(t)\right] t^{h_{1} r_{1}+\cdots+h_{m} r_{m}-r_{\lambda}+\omega+p+w_{2}}[t]^{*}
$$

when $t$ is in $W$. Here $w_{2}$ is taken the same for all of the involved terms in (5.4a) for $H=2 ; w_{2}$ is rational and $w_{2} \leqq 1$. It can be asserted that $c_{\lambda}(t)$ has the form (5.5) when $t$ is in $W$ (and $H=2$ ). Substitution of these forms in (5.4) (for $\boldsymbol{H}=2)$ will yield, in view of $(5.4 \mathrm{~b})$,

$$
\begin{aligned}
& { }_{2} \eta_{h_{1}}, \cdots, h_{m} ; j \\
& \left(h_{1}, \cdots, h_{m} \geqq 0 ; h_{1}+\cdots+h_{m}=2 ; j=1, \cdots, n ; t \text { in } W\right) .
\end{aligned}
$$

Here $w_{2}^{\prime}=\omega+p+w_{2}$. On taking account of (3.2) in view of (5.6) one may write

$$
\begin{gathered}
\nu_{r} \eta_{k_{1}}{ }^{r}, \cdots, k_{m}{ }^{r} ; \alpha \\
(\tau)=\exp \left[k_{1}{ }^{r} Q_{1}(\tau)+\cdots+k_{m}{ }^{r} Q_{m}(\tau)\right] \tau^{k_{1}{ }^{r} r_{1}+\cdots+k_{m}{ }^{r} r_{m}+\left(\nu_{r}-1\right) w_{2}{ }^{r}}[\tau]^{*} \\
\left(\tau \text { in } W ; k_{1}{ }^{r}, \cdots, k_{m}{ }^{r} \geqq 0 ; k_{1}{ }^{r}+\cdots+k_{m}{ }^{r}=\nu_{r} ; \alpha=1, \cdots, n ; \nu_{r}=1,2\right) .
\end{gathered}
$$

We shall now substitute (5.7) in (2.26) (for ${ }_{H}=3$ ). If the equalities of $(2.16 \mathrm{~b}$ ) and (5.7) are used it is inferred that

$$
\prod_{r=1}^{i_{\alpha}} \cdots=\exp \left[\delta_{1} Q_{1}(\tau)+\cdots+\delta_{m} Q_{m}(\tau)\right] \tau^{\delta_{1} r_{1}+\cdots+\delta_{m} r_{m}} \tau^{\nu\left(\gamma_{\alpha}, i_{\alpha}\right) w_{2}^{\prime}}[\tau]^{*}
$$


where

$$
\nu\left(\gamma_{\alpha}, i_{\alpha}\right)=\sum_{r=1}^{i_{\alpha}}\left(\nu_{r}-1\right)=\gamma_{\alpha}-i_{\alpha}
$$

The symbol

$$
\sum_{\nu_{1}+\cdots+\nu_{i_{\alpha}}=\gamma_{\alpha}} \sum_{k_{1}^{\prime}, \cdots, k_{m}^{\prime}}^{(2)} \quad\left(\delta_{i}={ }_{\alpha} \delta_{i}\right)
$$

(cf. (2.16b)) applied to the product (5.8) will yield a function

$$
F_{\alpha}^{\prime}=\text { generic form of the last member of (5.8) }
$$

In view of (2.24) and (2.19b) (where $\boldsymbol{H}=3$ )

$$
\begin{gathered}
{ }_{1} \delta_{q}+\cdots+{ }_{n} \delta_{q}=h_{q}(q=1, \cdots, m) ; \\
\gamma_{1}+\cdots+\gamma_{n}=3 ; i_{1}+\cdots+i_{n}=2,3 .
\end{gathered}
$$

Accordingly, in consequence of (5.8b) (with $\delta_{i}={ }_{\alpha} \delta_{i}$ ) and (5.8a), one has

$$
\begin{array}{r}
\prod_{\alpha=1}^{n} F_{\alpha}^{\prime}=\exp \left[h_{1} Q_{1}(\tau)+\cdots+h_{m} Q_{m}(\tau)\right] \tau^{h_{1} \tau_{2}}+\cdots+h_{m} r_{m} \tau^{(3-s)} w_{2}^{\prime}[\tau]^{*} \\
\left(s=i_{1}+\cdots+i_{n} ; \tau \text { in } W\right) .
\end{array}
$$

Since without any loss of generality one may consider that $w_{2}^{\prime} \geqq 0$ and since the only values that $s$ may assume (when ${ }_{H}=3$ ) are 2 and 3 , it is concluded that $\dagger$

$$
\prod_{\alpha=1}^{n} F_{\alpha}^{\prime}=\exp \left[h_{1} Q_{1}(\tau)+\cdots+h_{m} Q_{m}(\tau)\right] \tau^{h_{1} r_{1}+\cdots+h_{m} r_{m}} \tau^{w_{2}^{\prime}}[\tau]^{*}
$$

We next write

$$
\begin{aligned}
F_{i_{1}, \cdots, i_{n} ; \gamma_{1}, \cdots, \gamma_{n} i_{1} \delta_{1}, \cdots, \delta_{m} \delta_{m}}^{h_{1}, \cdots, h_{m}} & ={ }_{j} a_{i_{1}, \cdots, i_{n}} \prod_{\alpha=1}^{n} F_{\alpha}^{\prime} \\
& =\text { generic form of last member of (5.9). }
\end{aligned}
$$

Next it is noted that the first summation symbol of the last member of (2.26) is defined by (2.24), (2.19b); this summation is extended only over the subscripts of the left member of (5.10). The generic form displayed in (5.9) does not contain these subscripts. Hence, for $\tau$ in $W$,

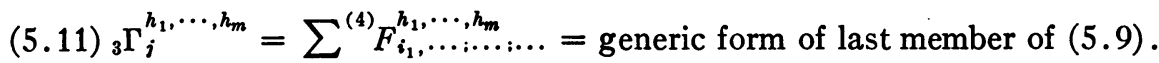

Assume now that, with a rational $w_{H-1}^{\prime} \geqq w_{2}^{\prime}\left(w_{H-1}^{\prime} \leqq \omega+p+1\right)$ and for $\tau$ in $W$, one has

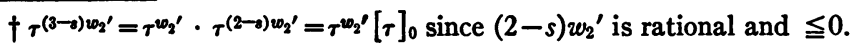




$$
\begin{array}{r}
{ }_{q+1} \Gamma_{j}^{h_{1}, \cdots, h_{m}}=\exp \left[h_{1} Q_{1}(\tau)+\cdots+h_{m} Q_{m}(\tau)\right] \tau^{h_{1} r_{1}+\cdots+h_{m} r_{m}} \tau^{(q-1) w_{H-1}^{\prime}}[\tau]^{*} \\
\left(h_{1}, \cdots, h_{m} \geqq 0 ; h_{1}+\cdots+h_{m}=q+1 ; j=1, \cdots, n\right), \\
{ }_{q} \eta_{k_{1}, \cdots, k_{m} ; \alpha}(\tau)=\exp \left[k_{1} Q_{1}(\tau)+\cdots+k_{m} Q_{m}(\tau)\right] \tau^{k_{1} r_{1}+\cdots+k_{m} r_{m}} \tau^{(q-1) w_{H-1}^{\prime}}[\tau]^{*} \\
\left(k_{1}, \cdots, k_{m} \geqq 0 ; k_{1}+\cdots+k_{m}=q ; \alpha=1, \cdots, n\right)
\end{array}
$$

for $q=1,2, \cdots, H-1(H \geqq 3)$. For $H=3$, that is for $q=1,2$, relations (5.12) and (5.13) have been established previously in (3.2), (3.5), (5.6), and (5.11).

Substitute (5.12) with $q=H-1$ in (5.4a). The integrand there displayed will be of the form

$$
e^{Q(\tau)} \tau^{r}[\tau]^{*} \quad(\tau \text { in } W),
$$

where

$$
\begin{aligned}
Q(\tau) & =h_{1} Q_{1}(\tau)+\cdots+h_{m} Q_{m}(\tau)-Q_{\lambda}(\tau), \\
r & =h_{1} r_{1}+\cdots+h_{m} r_{m}-r_{\lambda}+\omega+p+(B-2) w_{H-1}^{\prime}
\end{aligned}
$$

with

$$
h_{1}, \cdots, h_{m} \geqq 0 ; \quad h_{1}+\cdots+h_{m}=H
$$

In view of (5.14c) and (5.1) it is inferred that, for $\lambda>m$, one certainly has

$$
e^{Q(\tau)} \sim 0
$$

When $\lambda \leqq m, Q(\tau)$ will be a linear combination (with constant coefficients) of

$$
Q_{1}(\tau), \cdots, Q_{m}(\tau)
$$

only. With (3.10b) denoting the leading terms of the polynomials (5.15) it is recalled that $l^{\prime}$ was defined as the greater one of the numbers $l_{i} / k_{i}$ $(i=1, \cdots, m)$. Thus, when $\lambda \leqq m, Q(\tau)$ is of the form (3.10) and, unless $Q(\tau) \equiv 0$, there will be a leading term,

$$
q_{\nu_{1}} \tau^{\left(l-\nu_{1}\right) / k} \quad\left(q_{\nu_{1}} \neq 0 ; l-\nu_{1}>0\right),
$$

present in $Q(\tau)$; moreover, we shall have $\left(l-\nu_{1}\right) / k \leqq l^{\prime}$. It is accordingly seen that, when $\lambda \leqq m, Q(\tau)$ will be a polynomial of the variety of polynomials involved in the integration problem (4.1), (4.1a), (4.1b). On the other hand, when $\lambda>m$, since $\exp [Q(\tau)] \sim 0(\tau$ in $W)$, it is observed that $Q(t)$ will be a polynomial of the type which could occur in the problem $(4.29)-(4.29 \mathrm{c})$, provided one lets $R^{\prime}=W$. Thus Lemma $3, \S 4$ is applicable for evaluation of the integrals involved in (5.4a). With the aid of this lemma and in view of (5.14) we conclude that we may write

$$
\int^{t}(\text { integrand displayed in }(5.4 \mathrm{a}))=e^{Q(t)} t^{r+w_{B}}[t]^{*} \quad(t \text { in } w),
$$


where $w_{H}$ is rational and $w_{H} \leqq 1$. The functions $c_{\lambda}(t)$ (cf. (5.4a)) will be all of the generic form of the last member of (5.16). Hence, in view of (5.14a), (5.14b), one obtains from (5.4) and (5.4b)

$$
\begin{array}{r}
{ }_{H} \eta_{h_{1}, \cdots, h_{m} ; j}(t) \\
=\sum_{\lambda=1}^{n} \exp \left[h_{1} Q_{1}(t)+\cdots+h_{m} Q_{m}(t)\right] t^{h_{1} r_{1}+\cdots+h_{m} r_{m}+(H-2) w_{B-1}+w_{B}^{\prime \prime}}[t]^{*} \\
\quad\left(t \text { in } W ; h_{1}+\cdots+h_{m}=H ; j=1, \cdots, n\right),
\end{array}
$$

where $w_{H}^{\prime \prime}=\omega+p+w_{H} \leqq \omega+p+1$. Let $w_{H}^{\prime}$ be the greater of the numbers $w_{H}^{\prime \prime}$ and $w_{H-1}^{\prime}$. Necessarily $w_{H}^{\prime}$ will be rational and $w_{H}^{\prime} \leqq \omega+p+1$. On noting that

$$
t^{(H-2)\left(w_{H-1}^{\prime}-w_{H}^{\prime}\right)+w_{H}^{\prime \prime}-w_{H}^{\prime}}=[t]_{0},
$$

in view of (5.16a) it is inferred that, for $t$ in $W$

(5.17) $\quad H \eta_{h_{1}, \cdots, h_{m} ; j}(t)=\exp \left[h_{1} Q_{1}(t)+\cdots+h_{m} Q_{m}(t)\right] t^{h_{1} r_{1}+\cdots+h_{m} r_{m}+(H-1) w_{B}^{\prime}}[t]^{*}$.

This, however, implies that (5.13) holds also when $H$ is increased by unity; that is (5.13) holds for $q=1,2, \cdots, H$, provided that in (5.13) we write $w_{H}^{\prime}$ in place of $w_{H-1}^{\prime}$. This is possible for $q=1,2, \cdots, H-1$ because $w_{H-1}^{\prime}-w_{H}^{\prime}$ is rational and non-positive.

To determine the form of ${ }_{H+1} \Gamma_{j}^{h_{1}, \ldots, h_{m}}$ use will be made of (5.13), with $w_{H-1}^{\prime}$ replaced by $w_{H}^{\prime}$, and of (5.17). Substituting the known forms of the ${ }_{q} \eta \ldots ; j(t)(q=1,2, \cdots$,$) in (2.26), where { }_{H}$ is replaced by $H+1$, one obtains

(5.18) $\prod_{r=1}^{i_{\alpha}} \cdots=$ generic form of the last member of (5.8) ( $w_{2}^{\prime}$ replaced by $\left.w_{H}^{\prime}\right)$.

Here (5.8a) will hold. The summation symbol, displayed subsequent to (5.8a), applied to the product (5.18) will yield

(5.18a) $\quad F_{\alpha}^{\prime}=$ generic form of the last member of (5.18).

By virtue of the relations (2.24), (2.19b), where $\boldsymbol{H}$ is replaced by $\boldsymbol{H}+1$, one has

$$
\begin{array}{cc}
{ }_{1} \delta_{q}+\cdots+{ }_{n} \delta_{q}=h_{q} & (q=1, \cdots, m) ; \\
\gamma_{1}+\cdots+\gamma_{n}=H+1 ; i_{1}+\cdots+i_{n}=2,3, \cdots, H+1 .
\end{array}
$$

Thus from (5.18a), where we put $\delta_{i}={ }_{\alpha} \delta_{i}$, and in view of (5.8a) it is inferred that

$$
\begin{aligned}
& \prod_{\alpha=1}^{n} F_{\alpha}^{\prime}= \exp \left[h_{1} Q_{1}(\tau)+\cdots+h_{m} Q_{m}(\tau)\right] \tau^{h_{1} r_{1}+\cdots+h_{m} r_{m}} \tau^{(H+1-s) w_{H}^{\prime}}[\tau]^{*} \\
&= \exp \left[h_{1} Q_{1}(\tau)+\cdots+h_{m} Q_{m}(\tau)\right] \tau^{h_{1} r_{1}+\cdots+h_{m} r_{m}} \tau^{(H-1) w^{\prime}}[\tau]^{*} \\
&\left(s=i_{1}+\cdots+i_{n}=2,3, \cdots, B+1 ; \tau \text { in } W\right),
\end{aligned}
$$


since $w_{H}^{\prime} \geqq 0$ and $w_{H}^{\prime}$ is rational. The functions (5.10) will have the generic form of the last member of (5.19). In view of this fact

$$
\begin{aligned}
& H+1 \Gamma_{j}^{h_{1}, \cdots, h_{m}} \\
&=\sum^{(4)} F_{i_{1}, \cdots, i_{n} ; \gamma_{1}, \cdots, \gamma_{n} i_{1} \delta_{1}, \cdots, \delta_{n}}^{h_{1}, \cdots, h_{m}} \\
&=\exp \left[h_{1} Q_{1}(\tau)+\cdots+h_{m} Q_{m}(\tau)\right] \tau^{h_{1} r_{1}+\cdots+h_{m} r_{m}} \tau^{(H-1) w_{H}^{\prime}}[\tau]^{*}
\end{aligned}
$$

since the summation here involved is only with respect to the subscripts of $F$.

We accordingly conclude that relations (5.12) and (5.13) will be certainly valid for $H=3,4, \cdots$ when $\tau$ is in $W$. The numbers $w_{H}^{\prime}$ are rational and

$$
0 \leqq w_{2}^{\prime} \leqq w_{3}^{\prime} \leqq \cdots \leqq \omega+p+1 \text {. }
$$

Lemma 4. Let $R$ be a region of positive opening (cf. Definition 3, \$2), referred to in $\$ 2$ in connection with (i), (ii), (5.9a), (5.9b). As a matter of notation, within $R$ we have (5.1) and (5.1a), the $Q_{i}(\tau)(i=1, \cdots, n)$ being the polynomials involved in the formal matrix solution (2.5) of the linear differential system $\left(\mathrm{LA}_{1}\right)$, $\$ 2 . \dagger$ The highest power of $\tau$ actually present in $Q_{i}(\tau)$ being designated as $\tau^{i_{\mathbf{i}} / k_{i}}$ we let $l^{\prime}$ denote the greatest of the numbers $l_{i} / k_{i}(i=1, \cdots, m)$. Let $W$ be of the form $R\left(\alpha_{1}, \alpha_{2}\right)$ (cf. Definition 3), where

$$
0<\alpha_{2}-\alpha_{1}<\pi / l^{\prime},
$$

and be a subregion of $R$. The system (A), \$1 will possess a formal solution

$$
s_{j}(t)={ }_{1} y_{j}(t)+{ }_{2} y_{j}(t)+\cdots+{ }_{\nu} y_{j}(t)+\cdots \quad(j=1,2, \cdots, n),
$$

where, with $c_{1}, c_{2}, \cdots, c_{m}$ denoting arbitrary constants,

$$
\begin{aligned}
& { }_{\nu} y_{j}(t)=\sum_{k_{1}, \cdots, k_{m}} c_{1}^{k_{1} c_{2}^{k_{2}} \cdots c_{m}^{k_{m}}{ }_{\nu} \eta_{k_{1}, k_{2}}, \ldots, k_{m} ; j}(t) \\
& \left(k_{1}, \cdots, k_{m} \geqq 0 ; k_{1}+k_{2}+\cdots+k_{m}=\nu ; j=1, \cdots, n\right) .
\end{aligned}
$$

In (5.23a) the coefficients are functions which for $t$ in $W$, have the form

$$
{ }_{\nu} \eta_{k_{1}, \cdots, k_{m} ; j}(t)=\exp \left[k_{1} Q_{1}(t)+\cdots+k_{m} Q_{m}(t)\right] t^{k_{1} r_{1}+\cdots+k_{m} r_{m}} t^{(\nu-1) w_{\nu}}[t]_{q(\nu)}
$$

(cf. Definition 4, §2). $\ddagger$ The $w_{\nu}^{\prime}$ satisfy the conditions stated in connection with (5.21).

Note. A function $[t]_{q(\nu)}$ involved in the second member of (5.24) satisfies an asymptotic relation

$$
[t]_{q(v)} \sim\{t\}_{q(v)}
$$

$\dagger$ This system is identical with (LA), $\$ 2$ and is the linear system associated with the non-linear problem (A), \$1.

$\ddagger$ It might happen that $q(\nu) \rightarrow \infty$ as $\nu \rightarrow \infty$. 
(cf. Definition 1, §2). The formal expression $\{t\}_{q(\nu)}$ contains power series (generally divergent) of the form

$$
\rho_{0}+\rho_{1} t^{-1 / k_{\nu}}+\rho_{2} t^{-2 / k_{\nu}}+\cdots \quad\left(k_{\nu} \text { an integer }>0\right) .
$$

The $k_{\nu}(\nu=1,2, \cdots)$ may approach infinity with $\nu$.

In general the series (5.23) will diverge.

6. Transformations. With Lemma $4, \S 5$ in view, consider the transformation

$$
y_{j}(t)=Y_{j}(t)+\rho_{j}(t) \quad(j=1, \cdots, n),
$$

where

$$
Y_{j}(t)={ }_{1} y_{j}(t)+{ }_{2} y_{j}(t)+\cdots+{ }_{N-1} y_{j}(t) .
$$

Here the ${ }_{\nu} y_{j}(t)(\nu=1, \cdots, N-1 ; j=1, \cdots, n)$ are the functions (5.23a) and (5.24). $N$ is a fixed positive integer (which may be taken as large as desired). This transformation is to be applied to (A), $\$ 1$.

We let

$$
\left|c_{i}\right|<c^{\prime} \quad\left(c^{\prime}>0 ; i=1, \cdots, m\right) .
$$

Denoting the part of a region $G$ for which $|t| \geqq r^{\prime}$ by $G\left(|t| \geqq r^{\prime}\right)$ we observe that

$$
\left|Y_{j}(t)\right| \leqq \rho^{\prime} \quad\left[j=1, \cdots, n ; t \text { in } W\left(|t| \geqq r^{\prime}\right)\right],
$$

where

$$
0<\rho^{\prime}<\rho,
$$

where $\rho$ is the $\rho$ of (1.2), if $r^{\prime}$ (depending on $N$ and $c^{\prime}$ ) in (6.3) is taken sufficiently great. In order that (6.3) hold $r^{\prime}$ may be taken independent of $N$, provided $c^{\prime}$ (depending on $N$ ) of (6.2) has been taken sufficiently small.

Substitution of (6.1) in (A), $\$ 1$ will yield

$$
\begin{array}{r}
t^{-p} \rho_{j}^{(1)}(t)-l_{j}\left(t, \rho_{1}, \cdots, \rho_{n}\right)=-t^{-p} Y_{j}^{(1)}(t)+l_{j}\left(t, Y_{1}, \cdots, Y_{n}\right) \\
+q_{j}\left(t, Y_{1}+\rho_{1}, \cdots, Y_{n}+\rho_{n}\right) \quad(j=1, \cdots, n),
\end{array}
$$

where

$$
q_{j}\left(t, Y_{1}+\rho_{1}, \cdots\right)=q_{j}\left(t, Y_{1}, \cdots, Y_{n}\right)
$$

$$
+\sum_{\nu_{1}, \cdots, \nu_{n}} \alpha_{\nu_{1}, \nu_{2}, \cdots, \nu_{n} ; j}(t) \rho_{1}^{p_{1}^{1}}(t) \cdots \rho_{n}^{\nu_{n}}(t)
$$

$$
\left(\nu_{1} \geqq 0, \cdots, \nu_{n} \geqq 0 ; \nu_{1}+\nu_{2}+\cdots+\nu_{n}=1,2, \cdots\right),
$$

$$
\left.\nu_{1} ! \cdots \nu_{n} ! \alpha_{\nu_{1}}, \cdots, \nu_{n} ; j(t)=\frac{\partial^{\nu_{1}+\cdots+\nu_{n}}}{\partial^{\nu_{1}} y_{1} \cdots \partial^{\nu_{n}} y_{n}} q_{j}\left(t, y_{1}, \cdots, y_{n}\right)\right]
$$

$$
\left(y_{1}=Y_{1}(t), \cdots, y_{n}=Y_{n}(t)\right)
$$


(cf. (1.1b)). In view of (6.3) and the convergence conditions satisfied by the series (1.1b), the series (6.4a) with $j=1, \cdots, n$ are absolutely and uniformly convergent for $t$ in $W$, provided

$$
\left|\rho_{1}(t)\right|, \cdots,\left|\rho_{n}(t)\right| \leqq \rho^{\prime \prime}
$$

where

$$
0<\rho^{\prime \prime}<\rho-\rho^{\prime}
$$

with $\rho^{\prime}$ defined as in (6.3a).

We may write (6.4) in the form

$$
\begin{array}{r}
t^{-p} \rho_{j}^{(1)}(t)-l_{j}\left(t, \rho_{1}, \cdots, \rho_{n}\right)=\bar{q}_{j}\left(t, \rho_{1}, \cdots, \rho_{n}\right)-F_{j}(t) \\
\quad(j=1, \cdots n),
\end{array}
$$

where

$$
\bar{q}_{j}=\sum_{p_{1}+\cdots+v_{n} \geqq 1} \alpha_{v_{1}, \cdots, \nu_{n} ; j}(t) \rho_{1}^{n_{1}} \cdots \rho_{n}^{\eta_{n}}
$$

(cf. (6.4b)) and

$$
F_{j}(t)=t^{-p} Y_{j}^{(1)}(t)-l_{j}\left(t, Y_{1}, \cdots, Y_{n}\right)-q_{j}\left(t, Y_{1}, \cdots, Y_{n}\right) .
$$

To determine the form of $F_{j}(t)$ we first note that, by (2.23a),

$$
q_{j}\left(t, Y_{1}, \cdots, Y_{n}\right)=\sum_{H=2}^{\infty} \sum_{h_{1}+\cdots+h_{m}=H} c_{1}^{h_{1}} \cdots c_{m}^{h_{m}} \Gamma_{j}^{\prime h_{1}, \cdots, h_{m}}(t) .
$$

We now recall that the functions

$$
{ }_{H} \Gamma_{j}^{h_{1}, \cdots, h_{m}}(t),
$$

referred to in $\$ 5$, depend only on the functions

$$
{ }_{\nu} \eta_{k_{1}}, \cdots, k_{m} ; j(t)
$$

of rank less than $\boldsymbol{z}$ (cf. Definition 5 and (2.26)); the functions (6.9a) have in $W$ the form (5.24). It is clear that the function (6.8) can be given by the expression $q_{j}\left(t, y_{1}, \cdots, y_{n}\right)\left(y_{1}(t)=s_{1}(t), \cdots, y_{n}(t)=s_{n}(t)\right.$ the formal series (5.23)) provided that in the latter expression all the functions (6.9a) with rank equal to or greater than $N$ have been replaced by zeros. Hence, in (6.8), one has

$$
{ }_{H} \Gamma_{i}^{h_{1}, \cdots, h_{m}}(t)={ }_{H} \Gamma_{i}^{h_{1}, \cdots, h_{m}}(t) ;
$$

in the second member of (6.10) the functions (6.9a), for which $\nu \geqq N$, are replaced by 0 . In view of the statements in connection with (6.9), (6.9a), and (6.10) we have 


$$
{ }_{H} \Gamma_{j}^{h_{1}, \cdots, h_{m}}(t)={ }_{H} \Gamma_{j}^{h_{1}, \cdots, h_{m}}(t) \quad(B=2,3, \cdots, N) .
$$

For $H>N$ (6.10) will, in general, not hold. However, in view of (2.26) and in consequence of the indicated connection between the coefficients in (6.8) and the functions (6.9), it is inferred that, for $t$ in $W$,

$$
\begin{aligned}
{ }_{H} \Gamma_{j}^{\prime h_{1}, \cdots, h_{m}}(t) & =\text { generic form of }{ }_{H} \Gamma_{j}^{h_{1}, \cdots, h_{m}}(t) \\
& =\exp \left[h_{1} Q_{1}(t)+\cdots+h_{m} Q_{m}(t)\right] t^{h_{1} r_{1}+\cdots+h_{m} r_{m}} t^{(H-2) w_{B}^{\prime}-1}[t]^{*}
\end{aligned}
$$

for all values of the subscripts and superscripts (cf. (5.20) and (5.21)).

In view of the developments of $\$ 2$ and in particular in consequence of (2.13) and (2.14) we see that if in the second member of (6.7) one replaces the $Y_{i}$ by the $y_{i}(t)$ (formal solutions referred to in Lemma 4, §5), respectively, this member can be formally expanded as follows:

$$
\sum_{\nu=2}^{\infty} \sum_{k_{1}+\cdots+k_{m}=\nu} c_{1}^{k_{1}} \cdots c_{m}^{k_{m}} \lambda_{k_{1}, \cdots, k_{m} ; j}(t),
$$

where

$$
{ }_{\nu} \lambda_{k_{1}, \cdots, k_{m} ; j}(t)=t^{-p}{ }_{\nu}{ }_{\eta_{k_{1}}, \cdots, k_{m} ; j}^{(1)}(t)-l_{j}(t, \cdots)-{ }_{,} \Gamma_{j}^{k_{1}, \cdots, k_{m}}(t) .
$$

From the definition of the functions (6.9a) it follows that

$$
{ }_{\lambda} \lambda_{k_{1}}, \ldots, k_{m} ; j(t)=0
$$

for all values of the subscripts.

If in (6.11) the functions (6.9a) of rank $\geqq N$ are all replaced by zeros we obtain an expansion of (6.7),

$$
F_{j}(t)=\sum_{\nu=2}^{\infty} \sum_{k_{1}+\cdots+k_{m}=\nu} c_{1}^{k_{1}} \cdots c_{m}^{k_{m}}, \lambda_{k_{1}, \cdots, k_{m} ; j}^{\prime}(t),
$$

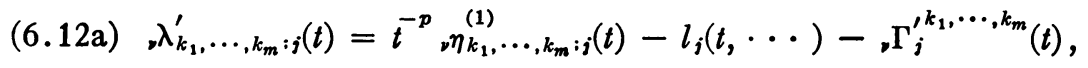

where the functions (6.9a) are replaced by zeros for $\nu \geqq N$. Thus, by (6.10a), (6.11a), and (6.11b),

$$
\begin{array}{lc}
{ }_{\nu} \lambda_{k_{1}, \cdots, k_{m} ; j}^{\prime}(t)={ }_{\nu} \lambda_{k_{1}, \cdots, k_{m} ; j}(t)=0 & (\nu=2,3, \cdots, N-1), \\
{ }_{\nu} \lambda_{k_{1}, \cdots, k_{m} ; j}^{\prime}(t)=-{ }_{\nu} \Gamma_{j}^{\prime k_{1}, \cdots, k_{m}}(t) & (\nu=N, N+1, \cdots) .
\end{array}
$$

Thus $F_{j}(t)$ is representable by

$$
-F_{j}(t)=\sum_{\nu \geqq N} \sum_{k_{1}+\cdots+k_{m}=\nu} c_{1}^{k_{1}} \cdots c_{m}^{k_{m}}, \Gamma_{j}^{\prime k_{1}, \cdots, k_{m}}(t) .
$$

In view of (5.21) one may replace $w_{H-1}^{\prime}$ in $(6.10 \mathrm{~b})$ by 


$$
\bar{\omega}=\omega+p+1 \text {. }
$$

Since

$$
[t]^{*} \leqq|t| \xi b_{\xi},
$$

where $\xi$ is positive, however small, the inequality being inferred from Definition $4, \S 2$, we accordingly obtain

$$
\left|{ }_{\nu} \Gamma_{j}^{\prime k_{1}, \cdots, k_{m}}(t)\right|<g_{k_{1}, \cdots, k_{m}}(t)|t|^{-2 \bar{\omega}+\xi} b_{!}^{\prime} \quad(t \text { in } W),
$$

where

$$
\begin{aligned}
g_{k_{1}}, \cdots, k_{m} & (t)=\left|\exp \left[k_{1} Q_{1}(t)+\cdots+k_{m} Q_{m}(t)\right] t^{k_{1} \bar{r}_{1}+\cdots+k_{m} \bar{r}_{m}}\right| \\
& \left(\bar{r}_{1}=r_{1}+\bar{\omega}, \cdots, \bar{r}_{m}=r_{m}+\omega\right) .
\end{aligned}
$$

Hence by (6.2) and (6.14) we have, for $t$ in $W$,

$$
\left|F_{j}(t)\right|<k_{0}\left(c^{\prime}\right)^{N}|t|^{-2 \bar{\omega}+\xi} \sum_{k_{1}+\cdots+k_{m}=N} g_{k_{1}, \cdots, k_{m}}(t)
$$

(cf. (6.16a) and (6.15)), where $k_{0}$ is a constant independent of $c^{\prime}$.

In the series (6.6a) the coefficients are given by (6.4b). In view of (1.1b) it accordingly follows that

$$
\alpha_{\nu_{1}, \cdots, v_{n} ; j}(t)=\sum_{\lambda_{1}, \cdots, \lambda_{n}}{ }_{j} a_{v_{1}+\lambda_{1}, \cdots, v_{n}+\lambda_{n}}(t) C_{\lambda_{1}}^{\nu_{1}+\lambda_{1}} \cdots C_{\lambda_{n}}^{\nu_{n}+\lambda_{n}} Y_{1}^{\lambda_{1}}(t) \cdots Y_{n}^{\lambda_{n}}(t)
$$

where

(6:18a) $\quad \lambda_{1} \geqq 0, \cdots, \lambda_{n} \geqq 0 ; \lambda_{1}+\cdots+\lambda_{n} \geqq 2-\left(\nu_{1}+\cdots+\nu_{n}\right)$.

Since, in $W, Y_{i}(t) \sim 0(i=1, \cdots, n)$ and since by $(6.18 \mathrm{a})$ for $\nu_{1}+\cdots+\nu_{n}=1$ we have $\lambda_{1}+\cdots+\lambda_{n} \geqq 1$, it is inferred from (6.18) that

$$
\alpha_{\nu_{1}}, \cdots, m ; j(t) \sim 0 \quad\left(t \text { in } W ; \nu_{1}+\cdots+\nu_{n}=1\right) .
$$

\section{Furthermore}

$$
\alpha_{\nu_{1}, \cdots, \nu_{n} ; j}(t)={ }_{j} a_{\nu_{1}, \cdots, \nu_{n}}(t)+{ }_{j} \beta_{\nu_{1}}, \cdots, \nu_{n}(t) \quad\left(\nu_{1}+\cdots+\nu_{n} \geqq 2\right),
$$

where

$$
{ }_{j} \beta_{v_{1}}, \cdots, v_{n}(t)=\sum_{\lambda_{1}+\cdots+\lambda_{n} \geqq 1} \cdots \sim 0 \quad(t \text { in } W) .
$$

By (6.19) equation (6.6) may be written in the form

$$
\begin{array}{r}
t^{-p} \rho_{j}^{(1)}(t)-l_{j}^{*}\left(t, \rho_{1}, \cdots, \rho_{n}\right)=q_{j}^{*}\left(t, \rho_{1}, \cdots, \rho_{n}\right)-F_{j}(t) \\
(j=1, \cdots, n),
\end{array}
$$

where 
(6.20a)

$$
q_{j}^{*}\left(t, \rho_{1}, \cdots, \rho_{n}\right)=\sum_{\nu_{1}+\cdots+\nu_{n} \geqq 2} \alpha_{\nu_{1}}, \cdots, \nu_{n} ; j(t) \rho_{1}^{\nu_{1}} \cdots \rho_{n}^{\nu_{n}}
$$

(cf. (6.19a), (6.19b)) and

$$
\left.l_{j}^{*}\left(t, \rho_{1}, \cdots, \rho_{n}\right) \sim l_{j}\left(t, \rho_{1}, \cdots, \rho_{n}\right) \quad \text { (in } t \text { for } t \text { in } W\right) . \dagger
$$

LEMMa 5. The transformation (6.1), (6.1a) applied to the non-linear system (A), $\$ 1$ will yield a system (6.20). In (6.20) $l_{j}^{*}$ is linear in $\rho_{1}, \cdots, \rho_{n}$, the coefficients of $\rho_{1}, \cdots, \rho_{n}$ being analytic, for $t \neq \infty$, in $W$; moreover, (6.20b) holds. The $q_{i}^{*}$ are given by (6.20a) and have coefficients analytic for $t$ in $W$ $(t \neq \infty)$. The series (6.20a) converge absolutely and uniformly (for $t$ in $W$ $\left(|t| \geqq r^{\prime}\right)$ and provided (6.2) is satisfied) whenever the inequalities (6.5), (6.5a) hold. The $F_{j}(t)$ are analytic in $W(t \neq \infty)$ and the $\left|F_{j}(t)\right|$ satisfy in $W$ inequalities (6.17). These assertions are made under the supposition that $\rho^{\prime}, r^{\prime}$, and $c^{\prime}$ (of (6.2)) have been selected so that inequalities (6.3a) and (6.3) hold.

7. The First Existence Theorem. We shall write for brevity

$$
q_{k_{1}, \cdots, k_{m} ; h}(t)=\exp \left[k_{1} Q_{1}(t)+\cdots+k_{m} Q_{m}(t)\right] t^{k_{1} \bar{r}_{1}+\cdots+k_{m} \bar{r}_{m}}
$$

and, with $\nu>0$,

$$
\left(r_{i}=r_{i}+h\right)
$$

$$
\begin{aligned}
q_{\nu: h}(t)= & \sum_{k_{1}, \cdots, k_{m}}\left|q_{k_{1}, \cdots, k_{m} ; h}(t)\right| \\
& \left(k_{1} \geqq 0, \cdots, k_{m} \geqq 0 ; k_{1}+\cdots+k_{m}=\nu\right) .
\end{aligned}
$$

If we recall that $Q_{i}(\tau)=q_{i} \tau^{l_{i} / k_{i}}+\cdots(i=1, \cdots, m)$, where $q_{i} \neq 0$ and $l_{i} / k_{i}>0$, and take account of

$$
R Q_{1}(\tau) \leqq R Q_{2}(\tau) \leqq \cdots \leqq R Q_{m}(\tau) \quad(\tau \text { in } R), \ddagger
$$

it is inferred that necessarily

$$
l^{\prime}=\frac{l_{1}}{k_{1}}=\frac{l_{2}}{k_{2}}=\cdots=\frac{l_{\bar{m}}}{k_{\bar{m}}}>\frac{l_{\bar{m}+1}}{k_{\bar{m}+1}} \geqq \cdots \geqq \frac{l_{m}}{k_{m}},
$$

where $1 \leqq \bar{m} \leqq m$. In fact, if the contrary were the case, for some $\nu(1 \leqq \nu<m)$ one would have

$$
\frac{l_{\nu}}{k_{\nu}}<\frac{l_{\nu+1}}{k_{\nu+1}}
$$

We shall write $\bar{\tau}=$ angle of $\tau$. Let $\bar{\tau}=\bar{\tau}_{0}$ be some ray, interior to $R$, with $\bar{\tau}_{0}$ $t$ in $W$.

$\dagger(6.20 \mathrm{~b})$ is to mean that $l_{j}^{*}-l_{j}=b_{1}(t) \rho_{1}+\cdots+b_{n}(t) \rho_{n}$, where $b_{i}(t) \sim 0$ for $i=1, \cdots, n$ and for

$\ddagger R$ is the region of Lemma $4, \S 5$. 
distinct from the limiting directions of the regular curves bounding $R$. Such a ray exists since by hypothesis $R$ is of positive opening. We have

$$
\begin{aligned}
& \lambda_{\nu}^{\prime}=\cos \left(\bar{q}_{\nu}+\frac{l_{\nu}}{k_{\nu}} \bar{\tau}_{0}\right)<0, \lambda_{\nu+1}^{\prime}=\cos \left(\bar{q}_{\nu+1}+\frac{l_{\nu+1}}{k_{\nu+1}} \bar{\tau}_{0}\right)<0 \\
&\left(\bar{q}_{i}=\angle q_{i} ; i=1, \cdots, m\right)
\end{aligned}
$$

since the above cosines can vanish only when $\bar{\tau}_{0}$ is replaced by the limiting directions of the regular curves

$$
R Q_{\nu}(\tau)=0, \quad R Q_{\nu+1}(\tau)=0,
$$

respectively, and since in $R$

$$
e^{Q_{\nu}(r)} \sim 0, \quad e^{Q_{\nu+1}(\tau)} \sim 0 .
$$

Now (7.1b) necessitates that along $\bar{\tau}=\bar{\tau}_{0}$ we should have

$$
\left|q_{\nu}\right| \lambda_{\nu}^{\prime}|\tau|^{l_{\nu} / k_{\nu}}+\cdots \leqq\left|q_{\nu+1}\right| \lambda_{\nu+1}^{\prime}|\tau|^{l_{\nu+1} / k_{\nu+1}}+\cdots
$$

that is,

$$
\left|q_{\nu}\right| \lambda_{\nu}{ }^{\prime}|\tau|^{-\alpha}+\cdots \leqq\left|q_{\nu+1}\right| \lambda_{\nu+1}^{\prime}+\cdots \quad(\alpha>0),
$$

which can be seen in view of (7.2a). In the limit, as $|\tau| \rightarrow \infty$ along the ray $\bar{\tau}=\bar{\tau}_{0}$, this will yield the inequality

$$
0 \leqq\left|q_{\nu+1}\right| \lambda_{\nu+1}^{\prime}
$$

which contradicts (7.3). Hence (7.2a) is impossible and the truth of (7.2) has been made evident.

DeFINITION 7. Let $R$ be a region of the type specified in Lemma 4, \$5. Let $W$ be a subregion of $R$ of the character indicated in the same lemma. Thus, $W=R\left(\alpha_{1}, \alpha_{2}\right)$ (cf. Definition 3, §2), where $0<\alpha_{2}-\alpha_{1}<\pi / l^{\prime}$ (cf. (7.2)). Let $W_{\xi}$ denote a subregion of $W$ of the form

$$
W_{\xi}=R\left(\alpha_{1}+\xi, \alpha_{2}-\xi\right) \quad(\xi>0 \text { and sufficiently small })
$$

where the two regular curves bounding $W_{\xi}$ are the rays

respectively. $\dagger$

$$
\angle t=\alpha_{1}+\xi, \quad \angle t=\alpha_{2}-\xi \quad\left(|t| \geqq r_{\xi}^{\prime}\right),
$$

In consequence of the definition of $W_{\xi}$ we have

$$
-\left|q_{i}\right| \cos \left(\bar{q}_{i}+\frac{l_{1}}{k_{1}} \bar{\tau}\right) \geqq \epsilon=\epsilon(\xi)>0 \quad(i=1, \cdots, \bar{m})
$$

for $\tau$ in $W_{\xi}$.

$\dagger$ The rest of the boundary of $W_{\xi}$ consists of an arc of the circle $|t|=r_{\xi}^{\prime}$. 
DEFINITION 8. If $G$ is a regular region such that, whenever $t$ is in $G$, all the points of the ray $\angle \tau=\angle t(|\tau| \geqq|t|)$ necessarily belong to $G$, and if $q(\tau)$ is positive and defined in $G$, it will be said that $q(\tau)$ is monotone in $G$, provided the upper bound of $q(\tau)(\angle \tau=\angle t ;|\tau| \geqq|t|)$ is attained at $t$ for every $t$ in $G$.

LemMa 6. Let $W_{\xi}$ be a region as given in Definition 7. With

$$
Q_{i}(\tau)=q_{i} \tau^{\nu^{\prime}}+\cdots \quad\left(\left|q_{i}\right| \neq 0 ; i=1, \cdots, \bar{m}\right)
$$

(cf. (7.2)) and with $\epsilon=\epsilon(\xi)$ denoting the number involved in (7.4a), let $\nu(\xi)$ be the least integer equal to or greater than

$$
\frac{\epsilon+\left|q_{\lambda}\right|}{\epsilon} \quad(\lambda=1, \cdots, \bar{m}-1) .
$$

Write

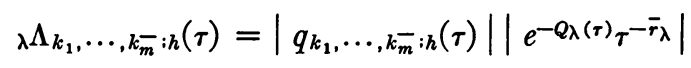

(cf. (7.1)), where

(7.6a) $\quad k_{1} \geqq 0, \cdots, k_{-} \geqq 0 ; \quad k_{1}+\cdots+k_{-}=\nu \geqq \nu(\epsilon) ; \quad \lambda=1,2, \cdots, n$.

There exists then a number $r^{\prime}=r^{\prime}(\xi, h)$, independent of $\lambda, k_{1}, \cdots, k_{\bar{m}}$, such that the functions (7.6) are all monotone (cf. Definition 8) in $W_{\xi}\left(|t| \geqq r^{\prime}\right)$.

To prove this lemma first take $r^{\prime}=r_{\xi, k}^{\prime}$ sufficiently great so that the functions

$$
\begin{array}{cr}
\left|e^{Q_{j}(\tau)} \tau^{\overline{r_{j}}}\right| & (j=1, \cdots, \bar{m}), \\
\left|\exp \left[Q_{i}(\tau)-Q_{\lambda}(\tau)\right] \tau^{\overline{r_{i}}-\overline{r_{\lambda}}}\right| & (i \leqq \bar{m} ; i<r)
\end{array}
$$

are all monotone in $W_{\xi}\left(|t| \geqq r^{\prime}\right)$. The possibility of such a choice follows without difficulty if we note that in $W_{\xi}$

$$
R Q_{j}(\tau)<0, \quad R\left(Q_{i}(\tau)-Q_{\lambda}(\tau)\right)<0
$$

for the values of the subscripts indicated in (7.7), (7.7a), while on the other hand the following is true. If $\bar{\tau}_{1}$ denotes the limiting direction of any one of the regular curves

$$
R Q_{j}(\tau)=0, \quad R\left(Q_{i}(\tau)-Q_{\lambda}(\tau)\right)=0 \quad(j \leqq \bar{m} ; i \leqq \bar{m} ; i<\lambda ;
$$

then, for $\tau$ (with $\angle \tau=\bar{\tau}$ ) in $W_{\xi}$, we have $\left|\bar{\tau}_{1}-\bar{\tau}\right| \geqq \zeta(\xi)>0$.

In view of the statement in connection with (7.7) we infer that the functions

$$
\left|q_{\nu_{1}}, \cdots, \overline{\nu_{m} ; h}(\tau)\right| \quad\left(\nu_{1} \geqq 0, \cdots, \nu_{\bar{m}} \geqq 0\right)
$$

(cf. (7.1)) are monotone in $W_{\xi}\left(|t| \geqq r^{\prime}\right), \dagger$ these functions being expressible

$\dagger$ The statement in connection with (7.8) can be made also when $\bar{m}$ is replaced by $m_{\text {: }}$ 
as products of non-negative powers of functions monotone in $W_{\xi}\left(|t| \geqq r^{\prime}\right)$. Let $\lambda>\bar{m}$. By (7.6a) some $k_{i}(i \leqq \bar{m})$ will be positive. Thus, if we write

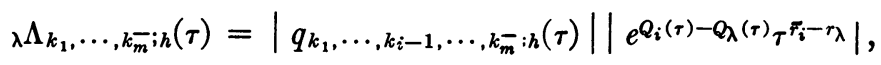

we observe that the statements made in connection with (7.8) and (7.7a) are applicable to the two factors of the second member of (7.9). Hence the functions (7.6) with $\lambda>\bar{m}$ are monotone in $W_{\xi}\left(|t| \geqq r^{\prime}\right)$.

Suppose $\lambda \leqq \bar{m}$ and $k_{\lambda}>0$. Then

$$
{ }_{\lambda} \Lambda_{k_{1}, \cdots, k_{m}^{-}: h}(\tau)=\left|q_{k_{1}, \cdots, k_{\lambda}-1, \cdots, k_{m}^{-} ; h}(\tau)\right| .
$$

The statement in connection with (7.8) is again applicable, and the functions (7.9a) $\left(\lambda \leqq \bar{m}, k_{\lambda}>0\right)$ are seen to be monotone in $W_{\xi}\left(|t| \geqq r^{\prime}\right)$.

Suppose $\lambda \leqq \bar{m}, k_{\lambda}=0$, and some $k_{i}>0(i<\lambda)$. We then write $(7.9)$ and demonstrate, as before, that the functions (7.6) are monotone in $W_{\xi}$ (under the conditions just stated).

It remains to consider the case when $\lambda \leqq \bar{m}$ and $k_{1}=k_{2}=\cdots=k_{\lambda}=0$. Necessarily one must have $\lambda<\bar{m}$ since otherwise $k_{1}+\cdots+k_{m}^{-}=0$, contrary to (7.6a). One may write

$$
{ }_{\lambda} \Lambda_{k_{1}}, \cdots, k_{m}^{-}: h(\tau)=\left|e^{Q(\tau)} t^{-\overline{r_{\lambda}}+h_{\lambda+1} \bar{r}_{\lambda+1}+\cdots+h_{\bar{m}} \overline{r_{m}}}\right| \cdot\left|q_{0, \cdots, 0 ; k_{\lambda+1}-h_{\lambda+1}, \cdots, k_{m}^{-}-h_{m}^{-}}(\dot{\tau})\right|,
$$

where

and

$$
\begin{aligned}
h_{\lambda+1} \geqq 0, \cdots, h_{m} \geqq 0 ; h_{\lambda+1}+\cdots+h_{\bar{m}}=\nu(\xi) & ; h_{i} \leqq k_{i} \\
& (i=\lambda+1, \cdots, \bar{m})
\end{aligned}
$$

$$
Q(\tau)=-Q_{\lambda}(\tau)+h_{\lambda+1} Q_{\lambda+1}(\tau)+\cdots+h_{\bar{m}} Q_{\bar{m}}(\tau) .
$$

Sets of integers $\left(h_{\lambda+1}, \cdots, h_{\bar{m}}\right)$, satisfying (7.10a) exist if $(7.6 \mathrm{a})$ is assumed (as it now is). Moreover, there is only a finite number of such sets. The second factor in the second member of (7.10) is monotone in $W_{\xi}$ in consequence of the statement in connection with (7.8). It will be necessary to consider the other factor. The real part of the function $(7.10 \mathrm{~b})$ is of the form

$$
R Q(\tau)=q(\bar{\tau})|\tau| l^{\prime}+\cdots,
$$

where

$$
\begin{aligned}
& -q(\bar{\tau})=\left|q_{\lambda}\right| \cos \left(\bar{q}_{\lambda}+l^{\prime} \bar{\tau}\right) \\
& \quad+\left[-h_{\lambda+1}\left|q_{\lambda+1}\right| \cos \left(\bar{q}_{\lambda+1} l^{\prime} \bar{\tau}\right)-\cdots-h_{\bar{m}}\left|q_{\bar{m}}\right| \cos \left(\bar{q}_{\bar{m}}+l^{\prime} \bar{\tau}\right)\right] .
\end{aligned}
$$

By (7.4a) and (7.10a)

$$
[\cdots] \geqq\left(h_{\lambda+1}+\cdots+h_{\bar{m}}\right) \epsilon=\nu(\xi) \epsilon .
$$


Thus from (7.11a), in view of the definition of $\nu(\xi)$ given in Lemma 6, we get

$$
-q(\bar{\tau}) \geqq \nu(\xi) \epsilon-\left|q_{\lambda}\right| \geqq \frac{\epsilon+\left|q_{\lambda}\right|}{\epsilon} \cdot \epsilon-\left|q_{\lambda}\right|=\epsilon \quad\left(\tau \text { in } W_{\xi}\right) .
$$

If (7.11) and (7.11b) are used it can be shown that

$$
R Q(\tau)=q(\bar{\tau})|\tau| l^{\prime}(1+\gamma(|\tau|, \bar{\tau}))|\tau|^{-\beta} \quad(\beta>0),
$$

where $|\gamma(|\tau|, \bar{\tau})|<\gamma\left(\tau\right.$ in $\left.W_{\xi}\right)$. Hence there exists a number

$$
r=r\left(\xi, h ; h_{\lambda+1}, \ldots, h_{m}^{-}\right)
$$

such that the factor of the second member of (7.10) is monotone in $W_{\xi}$ $(|t| \geqq r)$. By virtue of the italicized statement subsequent to $(7.10 \mathrm{~b})$ it is concluded that the lemma holds, with $r^{\prime}=r^{\prime}(\xi, h)$ defined as the greatest of all numbers (7.12) $\left(\lambda=1, \cdots, \bar{m}-1 ; h_{\lambda+1}, \cdots, h_{m}\right.$ such that $h_{\lambda+1} \geqq 0, \cdots, h_{m} \geqq 0$ and $\left.h_{\lambda+1}+\cdots+h_{m}=\nu(\xi)\right)$.

Consider now the functions (6.20a); they are represented by series satisfying the convergence conditions stated in Lemma 5, §6. Write

$$
\left|\rho_{i}\right| \leqq \rho^{*}, \quad\left|w_{i}\right| \leqq w^{*} \quad(i=1, \cdots, n),
$$

where

$$
\rho^{*}+w^{*} \leqq \rho^{\prime \prime} .
$$

We then have $\left|\rho_{i}\right| \leqq \rho^{\prime \prime},\left|\rho_{i}+w_{i}\right| \leqq \rho^{\prime \prime}(i=1, \cdots, n)$. With the conditions of Lemma 5 satisfied one may write

$$
\begin{aligned}
q_{j}^{*}\left(t, \rho_{1}+w_{1}, \cdots, \rho_{n}+w_{n}\right)-q_{j}^{*}\left(t, \rho_{1}, \cdots, \rho_{n}\right)=\sum_{i=1}^{n} \frac{\partial q_{j}^{*}}{\partial \rho_{i}} w_{i} \\
+\sum_{\nu_{1}, \cdots, \nu_{n}} q_{j: \nu_{1}, \cdots, \nu_{n}} w_{1}^{\nu_{1}} \cdots w_{n}^{\nu_{n}} \\
\quad\left(\nu_{1} \geqq 0, \cdots, \nu_{n} \geqq 0 ; \nu_{1}+\cdots+\nu_{n}=2\right),
\end{aligned}
$$

where the functions $q_{j ; \nu_{1}, \cdots, \nu_{n}}$ satisfy

$$
\left|q_{j: \nu_{1}, \cdots, \nu_{n}}\right| \leqq q^{\prime},
$$

provided (7.13) and (7.13a) hold. In view of the character of the coefficients $\alpha_{v_{1}}, \ldots, \nu_{n} j(t)$ in the series $(6.20 \mathrm{a})$, it is seen that $q^{\prime}$ can be selected as a constant independent of $t$. Since in (6.20a) $\nu_{1}+\cdots+\nu_{n} \geqq 2$ it follows that

$$
\frac{\partial q_{j}^{*}}{\partial \rho_{i}}=\sum_{k_{1}+\cdots+k_{n} \geqq 1} \alpha_{k_{1}, \cdots, k_{n}}^{i, j}(t) \rho_{1}^{k_{1}} \cdots \rho_{n}^{k_{n}}
$$

and, by (7.13), 


$$
\left|\frac{\partial q_{j}^{*}}{\partial \rho_{j}}\right|<q^{\prime \prime} \rho^{*} \quad\left(q^{\prime \prime} \text { independent of } t\right) .
$$

By (7.15), (7.15a), and (7.13)

$$
\left|q_{j}^{*}\left(t, \rho_{1}+w_{1}, \cdots\right)-q_{j}^{*}\left(t, \rho_{1}, \cdots\right)\right|<n q^{\prime \prime} \rho^{*} w^{*}+q\left(w^{*}\right)^{2}
$$

( $q$ independent of $t$ ). Thus the following is true.

Let $t$ be in $W\left(|t| \geqq r^{\prime}\right.$ ) (cf. Lemma 5, \$6). Assume that (7.13), (7.13a) are satisfied. Then

$$
\begin{aligned}
\mid q_{j}^{*}\left(t, \rho_{1}+w_{1}, \cdots, \rho_{n}+w_{n}\right)-q_{j}^{*}\left(t, \rho_{1}, \cdots,\right. & \left., \rho_{n}\right) \mid \\
& <\left(q^{\prime} \rho^{*}+q w^{*}\right) w^{*},
\end{aligned}
$$

where $q^{\prime}, q$ are independent of $t$. These inequalities will continue to hold when the $\rho_{i}$ and the $w_{i}$ are functions of $t$, provided that the inequalities (7.13), (7.13a) continue to hold.

With the aid of the systems

$$
\begin{array}{r}
t^{-p} \rho_{j: k}^{(1)}(t)-l_{j}^{*}\left(t, \rho_{1: k}, \cdots, \rho_{n: k}\right)=q_{j}^{*}\left(t, \rho_{1: k-1}, \cdots, \rho_{n: k-1}\right)-F_{j}(t) . \\
(j=1, \cdots, n ; k=0,1, \cdots),
\end{array}
$$

we shall seek to determine in succession the functions $\rho_{j: k}(j=1, \cdots, n$; $k=0,1, \cdots)$. In $(7.18)$ let

$$
\rho_{j:-1}=0 \quad(j=1, \cdots, n) .
$$

Write

$$
\rho_{j: k}-\rho_{j: k-1}=w_{j: k} \quad(j=1, \cdots, n ; k=0,1, \cdots) .
$$

Thus

$$
\rho_{j: k}=w_{j: 0}+w_{j: 1}+\cdots+w_{j: k} .
$$

The set (7.18) is equivalent to the sequence of systems

$$
\begin{aligned}
t^{-p} w_{j: 0}^{(1)}(t)-l_{j}^{*}\left(t, w_{1: 0}, \cdots, w_{n: 0}\right)= & -F_{j}(t) \quad(j=1, \cdots, n), \\
t^{-p} w_{j: k}^{(1)}(t)-l_{j}^{*}\left(t, w_{1: k}, \cdots, w_{n: k}\right)= & g_{j: k} \\
& (j=1, \cdots, n ; k=1,2, \cdots),
\end{aligned}
$$

where

$$
\begin{aligned}
g_{j: k}= & q_{j}^{*}\left(t, \rho_{1: k-2}+w_{1: k-1}, \cdots, \rho_{n: k-2}+w_{n: k-1}\right) \\
& -q_{j}^{*}\left(t, \rho_{1: k-2}, \cdots, \rho_{n: k-2}\right) .
\end{aligned}
$$

Under suitable convergence conditions the series 


$$
\rho_{j}(t)=w_{j: 0}+w_{j: 1}+\cdots \quad(j=1, \cdots, n)
$$

will constitute a set of "actual" solutions of the transformed system referred to in Lemma $5, \S 6$.

In view of $(6.20 \mathrm{~b})$ it follows that, from an asymptotic point of view, the solutions of the linear system, obtained from (6.20) by replacing the second members by zeros, are identical with the solutions of the system $\left(\mathrm{LA}_{1}\right), \S 2$. Thus the systems (7.21), (7.21a) may be written in the form

$$
w_{j: k}(t)=\sum_{\lambda=1}^{n} c_{\lambda}^{j: k}(t) e^{Q_{\lambda}(t)} t^{\lambda} \gamma^{\lambda, j}(t) \quad(j=1, \cdots, n ; k=0,1, \cdots),
$$

where

$$
c_{\lambda}^{j: k}(t)=\sum_{\lambda_{1}=1}^{n} \int^{t} g_{\lambda_{1}: k} e^{-Q_{\lambda}(\tau)} t^{-\lambda_{\lambda}+\omega+p} \bar{\gamma}^{\lambda_{1}, \lambda}(\tau) d \tau
$$

Here

$$
g_{j: 0}=-F_{j}(t)
$$

and

(7.23c) $\quad \gamma^{\lambda, j}(t)=[t]^{*}, \quad \bar{\gamma}^{\lambda_{1}, \lambda}(t)=[t]^{*}$ $(t$ in $W)$.

In view of $(7.23 \mathrm{c})$

$$
\left|\gamma^{\lambda, j}(t)\right|
$$

$$
\left|\bar{\gamma}^{\lambda_{1}, \lambda}(t)\right|<\left.|t|\right|^{\prime} \gamma
$$$$
\left(\epsilon^{\prime}>0 ; t \text { in } W\right) \text {. }
$$

Thus from (7.23) and (7.23a) it follows that, if $\left|g_{j ; k}\right| \leqq g_{k}(j=1, \cdots, n)$,

$$
\left|w_{j: k}(t)\right|<n \gamma^{2} \sum_{\lambda=1}^{n}\left|e^{Q_{\lambda}(t)} t^{r_{\lambda}+\epsilon^{\prime}}\right| \int^{t} g_{k}\left|e^{-Q_{\lambda}(\tau)} \tau^{-\lambda_{\lambda}+\bar{\omega}+2 \epsilon^{\prime}}\right|\left|\tau^{-1-\epsilon^{\prime}} d \tau\right|
$$

for $t$ in $W$ and provided the integrals involved in the second members of (7.24) exist.

Henceforth, unless the contrary is stated, $t$ and $\tau$ will be in $W_{\xi}$ (cf. Definition 7) and we shall suppose that all the previous results (including equations (7.21), (7.21a)) have been established with $m$ replaced by $\bar{m}$, where $\bar{m}$ is the integer occurring in (7.2). Moreover, we shall take $N \geqq \nu(\xi)$ (cf. Lemma 6).

By (7.1a), (7.1), and (6.17),

$$
\left|F_{j}(\tau)\right|<g_{0}^{*}(\tau)=k_{0}\left(c^{\prime}\right)^{N}|\tau|^{-2 \bar{\omega}+\xi} q_{N: \bar{\omega}}(\tau) \quad(\bar{\omega}=\omega+p+1) .
$$

In view of (7.25) and (7.23b) it is seen that the function, obtained by disregarding the factor $\left|\tau^{-1-\epsilon^{\prime}} d \tau\right|$ in the integrand displayed in (7.24) $(k=0)$, is less than

$$
\left[k_{0}\left(c^{\prime}\right)^{N}|\tau| \xi+2 \epsilon^{\prime}\right]\left[q_{N: \bar{\omega}}(\tau)\left|e^{-Q_{\lambda}(\tau)} \tau^{-\left(r_{\lambda}+\bar{\omega}\right)}\right|\right]=i_{\lambda}^{*}(\tau) \quad\left(\tau \text { in } W_{\xi}\right) .
$$


In consequence of (7.1a), (7.1)

$$
i_{\lambda}^{*}(\tau)=k_{0}\left(c^{\prime}\right)^{N} q_{N: h_{0}}(\tau)\left|e^{-Q_{\lambda}(\tau)} \tau^{-\left(r_{\lambda}+h_{0}\right)}\right|,
$$

where

$$
h_{0}=\bar{\omega}+\alpha_{0}, \quad \alpha_{0}=\left(\xi+2 \epsilon^{\prime}\right) /(N-1) .
$$

By (7.6)

$$
i_{\lambda}^{*}(\tau)=k_{0}\left(c^{\prime}\right)^{N} \sum_{k_{1}, \cdots, k_{\bar{m}}}{ }_{\lambda} \Lambda_{k_{1}, \cdots, k_{\bar{m}} ; h_{0}}(\tau) \quad\left(k_{1}+\cdots+k_{\bar{m}}=N \geqq \nu(\xi)\right) .
$$

From (7.26c), with the aid of Lemma 6 , it is inferred that $i_{\lambda}^{*}(\tau)$ is monotone (cf. Definition 8) in $W_{\xi}\left(|t| \geqq r^{\prime}\left(\xi, h_{0}\right)\right)$.

In the sequel, whenever $t$ is in $W_{\xi}$ and $\tau$ is the variable of integration, the path will be selected along the ray

$$
\angle \tau=\angle t \quad(|\tau| \geqq|t|) .
$$

The integral displayed in (7.24) (for $k=0$ ) is less than

$$
i_{\lambda}^{*}(t) \int^{t}|\tau|^{-1-\epsilon^{\prime}}|d \tau|=\frac{1}{\epsilon^{\prime}} i_{\lambda}^{*}(t)|t|^{-\epsilon^{\prime}}
$$

when $t$ is in $W_{\xi}\left(|t| \geqq r^{\prime}\left(\xi, h_{0}\right)\right)$. With the aid of (7.24) (for $\left.k=0\right),(7.27)$, and (7.26a) we obtain

$$
\begin{array}{r}
\left|w_{j: 0}(t)\right|<w_{0}^{*}(t)=w_{0}|t|^{-h_{0}} q_{N: h_{0}}(t), \quad w_{0}=\frac{n^{2} \gamma^{2}}{\epsilon^{\prime}} k_{0}\left(c^{\prime}\right)^{N} \\
\left(j=1, \cdots, n ; t \text { in } W_{\xi}\left(|t| \geqq r^{\prime}\left(\xi, h_{0}\right)\right) .\right.
\end{array}
$$

Write

$$
h_{1}=h_{0}+\alpha_{1}, \quad \alpha_{1}=\frac{1}{N}\left(-\alpha_{0}+2 \epsilon^{\prime}\right)
$$

(cf. $(7.26 \mathrm{~b}))$;

$$
w_{1}=\frac{n^{2} \gamma^{2}}{\epsilon^{\prime}} b \quad\left(b=q^{\prime}\left(1+\rho^{\prime \prime}\right)+q\right)
$$

(cf. (7.17));

$$
\Gamma(t)=w_{0} w_{1} q_{N: h_{1}}(t)
$$

(cf. (7.28), (7.1a), and (7.1)).

One will have

$$
w_{0}^{*}(t) \leqq \frac{\rho^{\prime \prime}}{1+\rho^{\prime \prime}}, \quad \Gamma(t) \leqq \frac{\rho^{\prime \prime}}{1+\rho^{\prime \prime}} \quad\left(t \text { in } W_{\xi}\left(|t| \geqq r^{*}\right)\right),
$$


where $r^{*}$ is some number $\geqq r^{\prime}\left(\xi, h_{1}\right), \geqq r^{\prime}\left(\xi, h_{0}\right)$; (7.31) can also be obtained for $t$ in $W_{\xi}\left(|t| \geqq r^{\prime}\left(\xi, h_{1}\right)\right)$ provided $c^{\prime}$ is taken sufficiently small.

Since (7.21b) (for $k=1),(7.19)$, and (7.28) are valid, and since, by (7.31), $w_{0}^{*}(t)<\rho^{\prime \prime}$, it follows from (7.17) that $\dagger$

$$
\left|g_{j: 1}(t)\right|<b\left(w_{0}^{*}(t)\right)^{2}=g_{1}^{*}(t) \quad\left(t \text { in } W_{\xi}\left(|t| \geqq r^{*}\right)\right) .
$$

It will now be proved that

$$
\left|w_{j: 1}(t)\right|<\Gamma(t) w_{0}^{*}(t)=w_{1}^{*}(t) \quad\left(t \text { in } W_{\xi}\left(|t| \geqq r^{*}\right)\right) .
$$

In fact, if we disregard the factor $\left|\tau^{-1-e^{\prime}} d \tau\right|$, the integrand displayed in (7.24) (for $k=1$ ) is less than

$$
\begin{array}{r}
b\left(w_{0}^{*}(\tau)\right)^{2}\left|e^{-Q_{\lambda}(\tau)} \tau^{-\tau_{\lambda}+\bar{\omega}+2 \epsilon^{\prime}}\right|=b w_{0}{ }^{2}|\tau|^{-h_{0}+\bar{\omega}+2 \epsilon^{\prime}} q_{N: h_{0}}(\tau) \\
\cdot\left[q_{N: h_{0}}(\tau) e^{-Q_{\lambda}(\tau)} \tau^{-r_{\lambda}-h_{0}}\right] .
\end{array}
$$

By (7.26b) and (7.29), $-h_{0}+\bar{\omega}+2 \epsilon^{\prime}=N \alpha_{1}$. Hence, for $h_{1}=h_{0}+\alpha$, one has

$$
|\tau|^{-h_{0}+\bar{\omega}+2 \epsilon^{\prime}} q_{N: h_{0}}(\tau)=q_{N: h_{1}}(\tau) .
$$

Thus the function of (7.34) is equal to

$$
b w_{0}^{2} q_{N: h_{1}}(\tau) \sum_{k_{1}+\cdots+k_{m}^{-}=N} \lambda \Lambda_{k_{1}}, \cdots, k_{m}^{-}: h_{0}(\tau) .
$$

In view of the inequalities satisfied by $r^{*}$ and in consequence of Lemma 6 it is observed that the functions (7.34a) $\lambda=1, \cdots, n$ are monotone in $W_{\xi}$ $\left(|t| \geqq r^{*}\right)$. Hence the integral displayed in (7.24) (for $\left.k=1\right)$ is less than the product of the function of (7.34a) (with $\tau$ replaced by $t$ ) by

$$
\int^{t}\left|\tau^{-1-\epsilon^{\prime}} d \tau\right|
$$

that is, by $\left(1 / \epsilon^{\prime}\right)|t|-\epsilon^{\prime}$. Making use of this fact, we infer from (7.24) (for $\left.k=1\right)$ that

$$
\left|w_{j: 1}(t)\right|<\frac{n \gamma^{2}}{\epsilon^{\prime}} \sum_{\lambda=1}^{n} b w_{0}^{2} q_{N: h_{1}}(t) \sum_{k_{1}+\cdots+k_{m}^{-}=N}{ }_{\lambda} \Lambda_{k_{1}}, \cdots, k_{m}^{-}: h_{0}(t) \cdot\left|e^{Q_{\lambda}(t)} t^{r} \lambda\right|=w_{1}^{*}(t) .
$$

By (7.6) and (7.29a)

$$
w_{1}^{*}(t)=\left(w_{0} w_{1}\right) q_{N: h_{1}}(t)\left(w_{0}|t|^{-h_{0}}\right) \sum_{k_{1}+\cdots+k_{m}^{-}=N} q_{k_{1}, \cdots, k_{m}^{-} ; h_{0}}(t) .
$$

With the aid of (7.30), (7.1a), and (7.28), the truth of (7.33) is deduced from this relation.

$\dagger$ In the second member of (7.32) $b$ could be replaced by $q(<b)$; this, however, is avoided in order to simplify subsequent developments. 
By (7.33) and (7.31)

$$
w_{1}^{*}(t) \leqq\left(\frac{\rho^{\prime \prime}}{1+\rho^{\prime \prime}}\right)^{2}, \quad w_{1}^{*}(t) \leqq w_{0}^{*}(t), \quad w_{0}^{*}(t)+w_{1}^{*}(t)<\rho^{\prime \prime}
$$

for $t$ in $W_{\xi}\left(|t| \geqq r^{*}\right)$.

In consequence of (7.21b) (for $k=2),(7.35)$, and (7.17)

$$
\begin{aligned}
\left|g_{j: 2}(t)\right|= & \left|q_{j}^{*}\left(t, w_{1: 0}(t)+w_{1: 1}(t), \cdots\right)-q_{i}^{*}\left(t, w_{1: 0}(t), \cdots\right)\right| \\
& <\left(q^{\prime} w_{0}^{*}(t)+q w_{1}^{*}(t)\right) w_{1}^{*}(t) \leqq b w_{0}^{*}(t) w_{1}^{*}(t)=g_{2}^{*}(t)
\end{aligned}
$$

for $t$ in $W_{\xi}\left(|t| \geqq r^{*}\right)$.

Assume now that for $t$ in $W_{\xi}\left(|t| \geqq r^{*}\right)$ and for $i=1,2, \cdots, k(\geqq 2)$ we have

$$
\begin{aligned}
\left|g_{j: i}(t)\right| & <g_{i}^{*}(t)=b w_{0}^{*}(t) w_{i-1}^{*}(t), \\
\left|w_{j: i-1}(t)\right| & <w_{i-1}^{*}(t)=\Gamma^{i-1}(t) w_{0}^{*}(t) \quad(j=1, \cdots, n) .
\end{aligned}
$$

In (7.32), (7.36), and (7.33) this has been established for $k=2$.

By (7.37) and (7.37a) (for $i=k$ )

$$
g_{k}^{*}(t)=b \Gamma^{k-1}(t)\left(w_{0}^{*}(t)\right)^{2} .
$$

Thus, by (7.37) (for $i=k$ )

$$
\begin{aligned}
& \left|g_{j: k}(\tau)\right|\left|e^{-Q_{\lambda}(\tau)} \tau^{-r_{\lambda}+\bar{\omega}+2 \epsilon^{\prime}}\right|<b \Gamma^{k-1}(\tau)\left(w_{0}^{*}(\tau)\right)^{2}\left|e^{-Q_{\lambda}(\tau)} \tau^{-r_{\lambda}+\bar{\omega}+2 \epsilon^{\prime}}\right| \\
& =b\left(w_{0} w_{1}\right)^{k-1} q_{N: h_{1}}^{k-1}(\tau) w_{0}^{2} q_{N: h_{0}}^{2}(\tau)\left|e^{-Q_{\lambda}(\tau)} \tau^{-\left(\lambda_{\lambda}+h_{0}\right)}\right|\left|\tau^{\bar{\omega}+2 \epsilon^{\prime}-h_{0}}\right|
\end{aligned}
$$

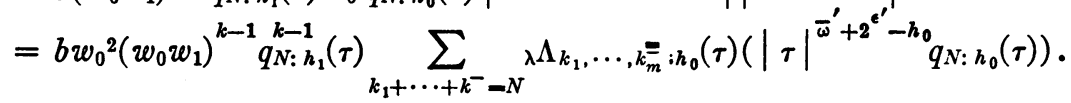

Making use of the formula subsequent to (7.34), one obtains

$$
\begin{aligned}
& \left|g_{j: k}(\tau)\right|\left|e^{-Q_{\lambda}(\tau)} \tau^{-r_{\lambda}+\bar{\omega}+2 \epsilon^{\prime}}\right| \\
& <b w_{0}^{k+1} w_{1}^{k-1} q_{N: h_{1}}^{k}(\tau) \sum_{k_{1}+\cdots+k_{m}^{-}=N}{ }^{k} \Lambda_{k_{1}, \cdots, k_{m} ; h_{0}}(\tau) .
\end{aligned}
$$

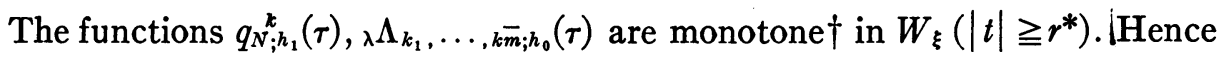
the integral displayed in (7.24) is less than the product of the second member of (7.38) (with $\tau$ replaced by $t$ ) by the function of $(7.34 \mathrm{~b})$, that is, by $\left(1 / \epsilon^{\prime}\right)$ $|t|^{-\epsilon^{\prime}}$. In view of this fact and (7.6) it is deduced from (7.24) that

$$
\left|w_{j: k}(t)\right|<\left(\frac{n^{2} \gamma^{2}}{\epsilon^{\prime}} b\right) w_{0}^{k+1} w_{1}^{k-1} q_{N: h_{1}}^{k}(t)|t|^{-h_{0}} \sum_{k_{1}+\cdots+k_{m}=N}\left|q_{k_{1}, \cdots, k^{-}: h_{0}}(t)\right| .
$$

By (7.29a) and (7.1a)

$$
\left|w_{j: k}(t)\right|<w_{k}^{*}(t)=\left(w_{0} w_{1} q_{N: h_{1}}(t)\right)^{k}\left(w_{0}|t|^{-h_{0}} q_{N: h_{0}}(t)\right)
$$

$\dagger$ Because $r^{*} \geqq r^{\prime}\left(\xi, h_{0}\right), r^{*} \geqq r^{\prime}\left(\xi, h_{1}\right)$. 
for $t$ in $W_{\xi}\left(|t| \geqq r^{*}\right)$ and for $j=1, \cdots, n$. Making use of (7.30) and (7.28) one obtains

$$
w_{k}^{*}(t)=\Gamma^{k}(t) w_{0}^{*}(t) .
$$

Furthermore, by (7.31), (7.37a) (for $i=1, \cdots, k$ ), and (7.40a)

$$
w_{i}^{*}(t) \leqq\left(\frac{\rho^{\prime \prime}}{1+\rho^{\prime \prime}}\right)^{i} w_{0}^{*}(t) \quad(i=0,1, \cdots, k) .
$$

Hence

$$
\rho_{k-1}^{*}(t)=w_{0}^{*}(t)+\cdots+w_{k-1}^{*}(t)<\left(1+\rho^{\prime \prime}\right) w_{0}^{*}(t)
$$

Also†

$$
w_{k}^{*}(t) \leqq\left(\frac{\rho^{\prime \prime}}{1+\rho^{\prime \prime}}\right)^{k} w_{0}^{*}(t)<w_{0}^{*}(t) \quad\left(t \text { in } W_{\xi}\left(|t| \geqq r^{*}\right)\right)
$$

and, by (7.31),

$$
\rho_{k-1}^{*}(t)+w_{k}^{*}(t) \leqq w_{0}^{*}(t) \sum_{i=0}^{k}\left(\frac{\rho^{\prime \prime}}{1+\rho^{\prime \prime}}\right)^{i}<\left(1+\rho^{\prime \prime}\right) w_{0}^{*}(t) \leqq \rho^{\prime \prime}
$$

By (7.21b) (cf. (7.21a)), in consequence of the statement in connection with (7.17),

$$
\begin{aligned}
\left|g_{j: k+1}(t)\right| & =\left|q_{j}^{*}\left(t, \rho_{1: k-1}(t)+w_{1: k}(t), \cdots\right)-q_{j}^{*}\left(t, \rho_{1: k-1}(t), \cdots, \rho_{n: k-1}(t)\right)\right| \\
& <\left(q^{\prime} \rho_{k-1}^{*}(t)+q w_{k}^{*}(t)\right) w_{k}^{*}(t) .
\end{aligned}
$$

Thus, by virtue of (7.43) and (7.42a), we have in $W_{\xi}\left(|t| \geqq r^{*}\right)$

$$
\left|g_{j: k+1}(t)\right|<\left[q !\left(1+\rho^{\prime \prime}\right) w_{0}^{*}(t)+q w_{0}^{*}(t)\right] w_{k}^{*}(t)=g_{k+1}^{*}(t) .
$$

Whence, in view of $(7.29 \mathrm{a})$,

$$
g_{k+1}^{*}(t)=b w_{0}^{*}(t) w_{k}^{*}(t) .
$$

In consequence of (7.40), (7.40a), (7.44), and (7.44a) it is observed that the relations (7.37), (7.37a) will necessarily hold when $k$ is replaced by $k+1$. It has therefore been proved that these relations hold for $k=2,3, \cdots$. Thus, in particular, in view of (7.31) one has

$$
\begin{aligned}
&\left|w_{j: k}(t)\right|<w_{k}^{*}(t) \leqq\left(\frac{\rho^{\prime \prime}}{1+\rho^{\prime \prime}}\right)^{k} w_{0}^{*}(t) \\
& \quad\left(j=1, \cdots, n ; k=0,1, \cdots ; t \text { in } W_{\xi}\left(|t| \geqq r^{*}\right)\right) .
\end{aligned}
$$

$\dagger$ (7.42), (7.42a) may be replaced by more precise inequalities and the developments could be given with a smaller $b$. 
Accordingly it is seen that the series (7.22) are absolutely and uniformly convergent, and

$$
\left|\rho_{j}(t)\right|<\left(1+\rho^{\prime \prime}\right) w_{0}^{*}(t)\left(\leqq \rho^{\prime \prime}\right) \quad(j=1, \cdots, n),
$$

when $t$ is in $W_{\xi}\left(|t| \geqq r^{*}\right)$.

From (7.21a), in view of (7.37), (7.37b), (7.40), (7.40a), we obtain

$$
\begin{gathered}
\left|t^{-p} w_{j: k}^{(1)}(t)\right|=\left|g_{j: k}(t)+l_{j}^{*}\left(t, w_{1: k}, \cdots, w_{n: k}\right)\right| \leqq\left|g_{j: k}(t)\right| \\
\quad+K \sum_{i=1}^{n}\left|w_{j: k}(t)\right|<\left[b \Gamma^{k-1}(t) w_{0}^{*}(t)+K n \Gamma^{k}(t)\right] w_{0}^{*}(t) .
\end{gathered}
$$

Thus, by (7.31),

$$
\left|t^{-p} w_{j: k}^{(1)}(t)\right|<b^{\prime}\left(\frac{\rho^{\prime \prime}}{1+\rho^{\prime \prime}}\right)^{k} w_{0}^{*}(t) \quad\left(b^{\prime}=b+K n\right) . \dagger
$$

Hence the derived series

$$
\sum_{k=0}^{\infty} w_{j: k}^{(1)}(t) \quad(j=1, \cdots, n)
$$

are absolutely and uniformly convergent in $W_{\xi}$. These series represent the derivatives of the functions $\rho_{j}(t)$ (involved in (7.46)), respectively. By (7.47)

$$
\left|\rho_{i}^{(1)}(t)\right|<b^{\prime}\left(1+\rho^{\prime \prime}\right) w_{0}^{*}(t)|t|^{p} \quad\left(t \text { in } W_{\xi}\left(|t| \geqq r^{*}\right)\right) .
$$

We are now ready to formulate the following existence theorem.

The First Existence Theorem. Let $R$ be a region referred to in $\$ 2$ in connection with (i), (ii), (7.9a), (7.9b). With $Q_{i}(\tau)(i=1, \cdots, n)$ denoting the polynomials involved in the formal matrix solution (2.5) of the linear system $\left(\mathrm{LA}_{1}\right), \S 2$, as a matter of notation we have in $R$

$$
R Q_{1}(t) \leqq R Q_{2}(t) \leqq \cdots \leqq R Q_{n}(t) ; \quad e^{Q_{i}(t)} \sim 0 \quad(i=1, \cdots, m) .
$$

Moreover, the $Q_{i}(\tau)$ will be supposed to be so selected that, on writing $Q_{i}(\tau)=q_{i} \tau^{l_{i} / k_{i}}+\cdots\left(i=1, \cdots, m ; q_{i} \neq 0\right)$, we have

$$
l^{\prime}=\frac{l_{1}}{k_{1}}=\frac{l_{2}}{k_{2}}=\cdots=\frac{l_{\bar{m}}}{k_{\bar{m}}}>\frac{l_{\bar{m}+1}}{k_{\bar{m}+1}} \geqq \cdots \geqq \frac{l_{m}}{k_{m}} \quad(1 \leqq \bar{m} \leqq m) .
$$

Let $W$ be a subregion of $R$ of the form $R\left(\alpha_{1}, \alpha_{2}\right)$ (cf. Definition 3, §2) where $0<\alpha_{2}-\alpha_{1}<\pi / l^{\prime}$. With $\xi>0$, however small, $W_{\xi}$ will denote a subregion of $W$

$\dagger$ Here $K$ is a number equal to or greater than the absolute values of the coefficients of the linear differential operators $l_{i}^{*}(j=1, \cdots, n)$; these coefficients, being asymptotically identical with the corresponding ones in the $l_{i}$ are therefore bounded for $t$ in $W_{\xi}$. 
of the form $W_{\xi}=R\left(\alpha_{1}+\xi, \alpha_{2}-\xi\right)$ (cf. Definition 7). Let $N$ be an integer $\geqq \nu(\xi)$, where $\nu(\xi)$ is the number defined in Lemma 6.

The non-linear system (A), $\$ 1$ will possess a solution $y_{j}(t)(j=1, \cdots, n)$, whose elements $y_{j}(t)$ are analytic for $t \neq \infty$ in $W_{\xi}\left(|t| \geqq r^{*}\right)$, while at $t=\infty$ they will generally be singular. Moreover,

$$
y_{j}(t) \sim s_{j}(t) \quad\left(j=1, \cdots, n ; t \text { in } W_{\xi}\left(|t| \geqq r^{*}\right)\right) .
$$

Here the $s_{j}(t)$ are the formal series given by (7.23), (7.23a), (7.24) of Lemma 4, \$5 under the supposition that in that lemma $m$ has been replaced $\dagger$ by $\bar{m}$. The asymptotic relations (7.50) have the following significance. The $y_{j}(t)$ are functions of the form

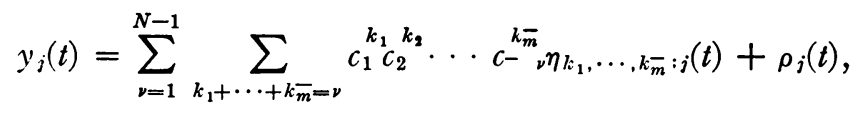

where $c_{1}, \cdots, c_{\bar{m}}$ are arbitrary constants $\left(\left|c_{i}\right| \leqq c^{\prime} ; i=1, \cdots, \bar{m}\right)$ and

$$
\begin{array}{r}
{ }_{\nu} \eta_{k_{1}}, \cdots, k^{-}: j \\
(t)=\exp \left(k_{1} Q_{1}(t)+\cdots+k_{\bar{m}} Q_{\bar{m}}(t)\right] t^{k_{1} r_{1}+\cdots+k_{\bar{m}} r_{\bar{m}} t^{(\nu-1)} w_{\nu}^{\prime}}[t]_{q(\nu)} \\
\left(t \text { in } W_{\xi}\left(|t| \geqq r^{*}\right)\right)
\end{array}
$$

(cf. Definition 4, §2, and (5.21)), where $w_{\nu}^{\prime} \leqq \omega+p+1$. Furthermore, the functions $\rho_{j}(t)=\rho_{j}\left(c_{1}, \cdots, c_{\bar{m}}, t\right)$ are analytic for $t \neq \infty$ in $W_{\xi}\left(|t| \geqq r^{*}\right)$ and satisfy in this region the inequalities

$$
\begin{aligned}
& \left|\rho_{j}(t)\right|<h^{\prime}\left(c^{\prime}\right)^{N}|t|^{(N-1) h_{0}} \\
& \quad \sum_{k_{1}+\cdots+k_{m}^{-}=N}\left|\exp \left[k_{1} Q_{1}(t)+\cdots+k_{\bar{m}} Q_{\bar{m}}(t)\right] t^{k_{0} r_{0}+\cdots+k_{m} r_{m}}\right|={ }_{N} \rho^{*}(t), \\
& \left|\rho_{j}^{(1)}(t)\right|<h^{\prime \prime}|t|_{N}^{p} \rho^{*}(t) \quad\left(j=1, \cdots, n ; h_{0}=\omega+p+1+\alpha_{0}\right)
\end{aligned}
$$

(cf. (7.26b)), provided $\left|c_{i}\right| \leqq c^{\prime}(i=1, \cdots, \bar{m})$. The above holds under the supposition that $c^{\prime}$ has been taken sufficiently small and that $r^{*}$ is sufficiently great.

Note. The term "asymptotic relations" applied to (7.50) is justifiable because in the neighborhood of $t=\infty$ the "remainder" term in (7.50a), that is to say, $\rho_{j}(t)$, is essentially of the same order of magnitude as

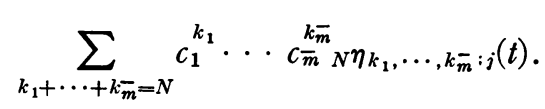

In fact, for $t$ in $W_{\xi}\left(|t| \geqq r^{*}\right)$, the absolute value of the above function is less than

$$
h^{\prime \prime}|t|^{-\xi-2 \epsilon^{\prime}}{ }_{N} \rho^{*}(t) .
$$

We consider it extremely likely that facts of the type of those stated in the

$\dagger$ This obviously has no effect on $l^{\prime}$. 
above theorem will hold in the region $W$ itself, even if in the formal series (involved in Lemma $4, \S 5) m$ is not replaced by $\bar{m}$, this being true at least when $W$ is subject to the condition that its opening (cf. Definition 3, §2) is less than $\pi /\left(2 l^{\prime}\right)$. An attempt to prove this conjecture leads to analytic difficulties even greater than those encountered so far.

8. Formal solutions for systems (B). The First Existence Theorem (\$7) is of course applicable to systems (B), \$1. However, in view of the specialized (as compared with systems (A), \$1) character of these systems, results of a more precise character, valid in more extensive regions, can be obtained by treating (B) directly. We have

$$
\begin{aligned}
& y_{j}^{(1)}(t)=a_{j}\left(y_{1}, \cdots, y_{n}\right) \quad(j=1, \cdots, n), \\
& a_{j}\left(y_{1}, \cdots, y_{n}\right)=l_{j}\left(y_{1}, \cdots, y_{n}\right)+q_{j}\left(y_{1}, \cdots, y_{n}\right), \\
& l_{j}\left(y_{1}, \cdots, y_{n}\right)=l_{1, j} y_{1}+\cdots+l_{n, j} y_{n}, \\
& q_{j}\left(y_{1}, \cdots, y_{n}\right)=\sum_{j} a_{i_{1}, \cdots, i_{n}} y_{1}^{i_{1}} \cdots y_{n} \\
&\left(i_{1}, i_{2}, \cdots, i_{n} \geqq 0 ; i_{1}+\cdots+i_{n} \geqq 2 ; j=1, \cdots, n\right),
\end{aligned}
$$

the series in the second member of (8.1b) being convergent for

$$
\left|y_{1}\right|, \cdots,\left|y_{n}\right| \leqq \rho . \dagger
$$

Associated with (B) there is a linear system

$$
y_{j}^{(1)}(t)=l_{j}\left(y_{1}, \cdots, y_{n}\right) \quad(j=1, \cdots, n)
$$

which is equivalent to the matrix equation

$$
Y^{(1)}(t)=Y(t) B, \quad Y(t)=\left(y_{i, j}(t)\right),
$$

where

$$
B=\left(l_{i, j}\right) \quad(i, j=1, \cdots, n) .
$$

Here elements of a row in $Y(t)$ will constitute a solution of (LB).

The characteristic equation $\ddagger$

$$
E(\alpha)=\left|\left(l_{i, j}-\delta_{i, j} \alpha\right)\right|=0
$$

has roots

$$
\alpha_{1}, \alpha_{2}, \cdots, \alpha_{\lambda} \quad\left(1 \leqq \lambda \leqq n ; \alpha_{k} \neq \alpha_{\nu} \text { for } k \neq \nu\right)
$$

of multiplicities

$$
m_{1}, m_{2}, \cdots, m_{\lambda} \quad\left(m_{1}+m_{2}+\cdots+m_{\lambda}=n\right),
$$

$\dagger$ The same remark is made as in the footnote in connection with (1.2).

$\ddagger$ Here $\left(\delta_{i, j}\right)$ is the identity matrix; i.e., $\delta_{i, j}=0$, for $i \neq j$, and $\delta_{i, i}=1$. 
respectively. The equation $\left(\mathrm{LB}_{1}\right)$ will possess a matrix solution

$$
Y(t)=\left(e^{q_{i} t} p_{i, j}(t)\right) \quad(i, j=1, \cdots, n),
$$

where

$$
\begin{array}{rr}
q_{i}=\alpha_{1}\left(i=1, \cdots, m_{1}\right) ; & q_{i}=\alpha_{2}\left(i=m_{1}+1, \cdots, m_{1}+m_{2}\right) ; \cdots, \\
q_{i}=\alpha_{\lambda} & \left(i=m_{1}+\cdots+m_{\lambda-1}+1, \cdots, n\right),
\end{array}
$$

and the $p_{i, j}(t)$ are polynomials in $t$ of degree at most

$$
\begin{aligned}
& m_{1}-1 \quad\left(\text { for } i=1, \cdots, m_{1}\right), \\
& m_{2}-1 \quad\left(\text { for } i=m_{1}+1, \cdots, m_{1}+m_{2}\right) \text {, } \\
& \left.m_{\lambda-1}-1 \text { (for } i=m_{1}+\cdots+m_{\lambda-1}+1, \cdots, n\right) \text {. }
\end{aligned}
$$

It will be assumed that not all of the roots of the characteristic equation (8.3) are zero. Thus in (8.4) at least one of the $q_{i}$ will be distinct from zero.

In the sequel there will be occasion to consider the matrix

$$
Y^{-1}(t)=\bar{Y}(t) .
$$

Since it satisfies the equation

$$
\bar{Y}^{(1)}(t)=-B \bar{Y}(t),
$$

whose characteristic equation is $E(-\alpha)=0$ (cf. (8.3a)), if we take account of the interchanged role of the rows and columns we conclude that

$$
Y^{-1}(t)=\left(\bar{y}_{i, j}(t)\right)=\left(\bar{p}_{i, j}(t) e^{-q_{j} t}\right)
$$

where the $\bar{p}_{i, j}(t)$ are polynomials in $t$ of degrees at most as stated in (8.4b) (with $i$ replaced by $j$ ). Moreover,

$$
\left(\bar{p}_{i, j}(t)\right)\left(p_{i, j}(t)\right)=\left(\delta_{i, j}\right)=I .
$$

Definition 9. $S\left(\alpha_{1}, \alpha_{2} ; r\right)$ will denote the sector

$$
\alpha_{1} \leqq \angle t \leqq \alpha_{2} \quad(|t| \geqq r) .
$$

Let the $B_{i}$ denote the rays along which $R\left(q_{i} t\right)=0$ (whenever the constant $q_{i}$, under consideration, $\neq 0$ ). Let

$$
S_{1}, S_{2}, \cdots
$$

denote the sectors separated by the $B_{i}$ rays and not containing any of these rays in their interiors.

In the above set of sectors (8.6) there is certain to exist at least one sector $S$ such that the following holds. 
In the set of numbers $q_{i}(i=1, \cdots, n)$ there are some, say

$$
q_{i_{1}}, q_{i_{2}}, \cdots, q_{i_{n}}
$$

$$
(1 \leqq m \leqq n)
$$

all distinct from zero, corresponding to which

$$
R\left(q_{i_{\tau}} t\right) \leqq 0
$$$$
(\tau=1, \cdots, m ; t \text { in } S) .
$$

As a matter of notation the functions $q_{i}$ involved in (8.7a) will be designated as

$$
q_{1} t, q_{2} t, \cdots, q_{m} t .
$$

Henceforth, unless the contrary is stated or implied, we consider a particular sector $S$ of the above description. It will be designated as

$$
S\left(\alpha_{1}, \alpha_{2} ; r\right)
$$

$$
\left(\alpha_{1}<\alpha_{2}\right)
$$

(cf. Definition 9).

Let $c_{1}, \cdots, c_{m}$ denote arbitrary constants, and consider a solution of (LB) of the form

$$
{ }_{1} y_{j}(t)=\sum_{\lambda=1}^{m} c_{\lambda} e^{q_{\lambda} t} p_{\lambda, j}(t) \quad(j=1, \cdots, n) .
$$

We shall seek to satisfy the non-linear system (B) with the aid of the formal series

$$
y_{j}(t)={ }_{1} y_{j}(t)+{ }_{2} y_{j}(t)+\cdots+{ }_{\nu} y_{j}(t)+\cdots \quad(j=1, \cdots, n),
$$

where

$$
\begin{aligned}
& { }_{\nu} y_{j}(t)=\sum_{k_{1}, \cdots, k_{m}} c_{1}^{k_{1}} \cdots c_{m}^{k_{m}}{ }_{\nu} \eta_{k_{1}}, \cdots, k_{m} ; j(t) \\
& \left(k_{1}, \cdots, k_{m} \geqq 0 ; k_{1}+\cdots+k_{m}=\nu\right) \text {. }
\end{aligned}
$$

For $\nu=1$ the coefficients in (8.10a) are already known. In fact, by (8.9),

$$
e^{q_{\lambda} t} p_{\lambda, j}(t)={ }_{1} \eta_{k_{1}}, \cdots, k_{m} ; j(t) \quad(\lambda=1, \cdots, m ; j=1, \cdots, n),
$$

where $k_{i}=0$ for $i \neq \lambda$ and $k_{\lambda}=1$.

On substituting (8.10), (8.10a) in (B) it is found that the coefficients involved in (8.10a) satisfy the linear non-homogeneous systems $\left(S_{H}\right), \S 2$ $(\mathrm{H}=2,3, \cdots)$. Furthermore, in place of $(2.30)$ and $(2.30 \mathrm{a})$ we now deduce in consequence of (8.5a) and (8.4)

$$
{ }_{H} \eta_{h_{1}}, \cdots, h_{m} ; j(t)=\sum_{\lambda=1}^{n} c_{\lambda}(t) e^{q_{\lambda} t} p_{\lambda, j}(t),
$$

where 


$$
c_{\lambda}(t)=\sum_{\lambda_{1}=1}^{n} \int_{H}^{t} \Gamma_{\lambda_{1}}^{h_{1}, \cdots, h_{m}}(\tau) \bar{p}_{\lambda^{\prime}, \lambda}(\tau) e^{-q_{\lambda} \tau} d \tau
$$

Here the

$$
{ }_{H} \Gamma_{\lambda_{1}}^{h_{1}, \cdots, h_{m}}(\tau)
$$

are given by (2.26).

LEMMA 7. Consider the problem (B) at the beginning of this section. Let $Y(t)$, as given by (8.4), be a matrix solution of $\left(\mathrm{LB}_{1}\right)$. A mechanism for construction of formal solutions (8.10) (cf. (8.10a)) of the system (B) is given by (8.11), (8.11a) (cf. (8.5a) and (2.26)).

It will be convenient to introduce the following definition.

Definitron 10. Generically $[t, \nu]$ is to denote a polynomial in $t$ of at most the vth degree.

By (8.10b)

$$
{ }_{1} \eta_{k_{1}^{r}, \cdots, k_{m}^{r} ; \alpha}^{r}(\tau)=\exp \left[\left(k_{1}^{r} q_{1}+\cdots+k_{m}^{r} q_{m}\right) \tau\right]\left[\tau, m^{\prime}\right]
$$

( $m^{\prime}$ the greater of the numbers $(8.4 \mathrm{~b})$ ). In view of (8.12) the left member of (3.3a) becomes

$$
\exp \left[\left(\delta_{1} q_{1}+\cdots+\delta_{m} q_{m}\right) \tau\right]\left[\tau, i_{\alpha} m^{\prime}\right] .
$$

Applying the summation displayed subsequent to (3.3a) to (8.13), we get

$$
F_{\alpha}^{\prime}=\text { generic form of (8.13). }
$$

In view of (2.24), (2.19b) we have

$$
\prod_{\alpha=1}^{n} F_{\alpha}^{\prime}=\exp \left[\left(h_{1} q_{1}+\cdots+h_{m} q_{m}\right) \tau\right]\left[\tau, s m^{\prime}\right] \quad\left(s=i_{1}+\cdots+i_{n}\right)
$$

By (3.4)

$$
\begin{aligned}
{ }_{2} \Gamma_{i}^{h_{1}, \cdots, h_{m}}(\tau) & =\sum_{i_{1}+\cdots+i_{n}=2} \sum_{\gamma_{1}+\cdots+\gamma_{n}=2} \sum_{{ }_{1} \delta_{1}, \cdots, n^{\delta_{m}}}^{(3)}{ }_{j} a_{i_{1}, \cdots, i_{n}} \prod_{\alpha=1}^{n} F_{\alpha}^{\prime} \\
& =\exp \left[\left(h_{1} q_{1}+\cdots+h_{m} q_{m}\right) \tau\right]\left[\tau, 2 m^{\prime}\right] .
\end{aligned}
$$

Since

$$
\bar{p}_{i, j}(\tau)=\left[\tau, m^{\prime}\right] \quad(i, j=1, \cdots, n)
$$

in consequence of (8.14), it is observed that the integrand displayed in (8.11a) is of the form

$$
\exp \left[\left(h_{1} q_{1}+\cdots+h_{m} q_{m}-q_{\lambda}\right) \tau\right]\left[\tau, 3 m^{\prime}\right] .
$$

Hence the integral displayed in (8.11a) is expressible as 


$$
\exp \left[\left(h_{1} q_{1}+\cdots+h_{m} q_{m}-q_{\lambda}\right) t\right]\left[t, 3 m^{\prime}+1\right],
$$

where $3 m^{\prime}+1$ may be replaced by $3 m^{\prime}$ whenever $h_{1} q_{1}+\cdots+h_{m} q_{m}-q_{\lambda} \neq 0$. One further finds that $c_{\lambda}(t)$ has the generic form of (8.14b). Hence, by (8.11) (for $\mathrm{H}=2$ ),

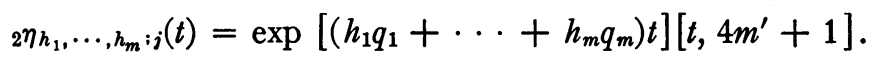

In view of (8.12) and (8.15)

$$
\begin{aligned}
{ }_{\nu} \eta_{k_{1}}, \cdots, k_{m} ; \alpha & (t)=\exp \left[\left(k_{1} q_{1}+\cdots+k_{m} q_{m}\right) \tau\right]\left[\tau, \rho_{\nu}\right] \\
& \left(\rho_{\nu}=\nu\left(3 m^{\prime}+1\right)-\left(2 m^{\prime}+1\right) ; \nu=1,2\right) .
\end{aligned}
$$

On writing $k_{i}=k_{i}{ }^{r}(i=1, \cdots, m), \nu=\nu_{r}$, substitute (8.16) in (2.26) (for $\mathrm{H}=3$ ). The product (5.8) will now be of the form

$$
\exp \left[\left(\delta_{1} q_{1}+\cdots+\delta_{m} q_{m}\right) \tau\right]\left[\tau, \rho_{\alpha}^{\prime}\right],
$$

where

$$
\rho_{\alpha}^{\prime}=\sum_{r=1}^{i \alpha} \rho_{\nu}=\gamma_{\alpha}\left(3 m^{\prime}+1\right)-i_{\alpha}\left(2 m^{\prime}+1\right)
$$

(cf. (5.8a)). Applying to (8.17) the summation symbol subsequent to (5.8a) one obtains

$$
F_{\alpha}^{\prime}=\text { generic form of (8.17). }
$$

In view of $(5.8 \mathrm{c})$

$$
\prod_{\alpha=1}^{n} F_{\alpha}^{\prime}=\exp \left[\left(h_{1} q_{1}+\cdots+h_{m} q_{m}\right) \tau\right]\left[\tau, \rho_{s}^{\prime \prime}\right]
$$

where, by $(8.17 \mathrm{a})$,

$$
\rho_{s}^{\prime \prime}=\sum \rho_{\alpha}^{\prime}=3\left(3 m^{\prime}+1\right)-s\left(2 m^{\prime}+1\right) \quad\left(s=i_{1}+\cdots+i_{n}\right) .
$$

Here $s$ may assume only the values 2 and 3 . Hence in (8.18a) we may replace $\left[\tau, \rho_{s}^{\prime \prime}\right]$ by $\left[\tau, 5 m^{\prime}+1\right]$. Thus the functions involved in the left member of (5.10) will possess the generic form of $(8.18 \mathrm{a})$ (where the indicated replacement has been made). If now the first summation symbol of the last member in (2.26) is extended over the functions (5.10), it is seen that the generic form will remain unaltered. Thus

$$
{ }_{3} \Gamma_{j}^{h_{1}, \cdots, h_{m}}=\exp \left[\left(h_{1} q_{1}+\cdots+h_{m} q_{m}\right) \tau\right]\left[\tau, 5 m^{\prime}+1\right] .
$$

Assume that, with $\rho_{q}=q\left(3 m^{\prime}+1\right)-\left(2 m^{\prime}+1\right)$, 


$$
\begin{aligned}
&{ }_{q+1} \Gamma_{j}^{h_{1}, \cdots, h_{m}}=\exp \left[\left(h_{1} q_{1}+\cdots+h_{m} q_{m}\right) \tau\right]\left[\tau, \rho_{q}+m^{\prime}\right] \\
&\left(h_{1}, \cdots, h_{m} \geqq 0 ; h_{1}+\cdots+h_{m}=q+1 ; j=1, \cdots, n\right),
\end{aligned}
$$

and

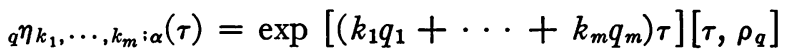

$$
\begin{aligned}
& \left(k_{1}, \cdots, k_{m} \geqq 0 ; k_{1}+\cdots+k_{m}=q ; \alpha=1, \cdots, n\right)
\end{aligned}
$$

for $q=1,2, \cdots, \mathrm{H}-1(\mathrm{H} \geqq 3)$.

Substitute (8.20) (for $q={ }_{B}-1$ ) in (8.11a). The integrand involved in (8.11a) will be of the form

$$
\exp \left[\left(h_{1} q_{1}+\cdots+h_{m} q_{m}-q_{\lambda}\right) \tau\right]\left[\tau, \rho_{H-1}+2 m^{\prime}\right] .
$$

Thus the integral displayed in (8.11a) is

$$
\exp \left[\left(h_{1} q_{1}+\cdots+h_{m} q_{m}-q_{\lambda}\right) t\right]\left[t, \rho_{H-1}+2 m^{\prime}+1\right] .
$$

Here $2 m^{\prime}+1$ may be replaced by $2 m^{\prime}$ whenever $h_{1} q_{1}+\cdots+h_{m} q_{m}-q_{\lambda} \neq 0$. Whence $c_{\lambda}(t)$, as given by (8.11a), will be of the form (8.21). Finally, from (8.11) one obtains (8.20a) with $q$ replaced by н. By making use of the relations (8.20a) (for $q=1, \cdots, \mathrm{H}$ ) we are going to prove that (8.20) holds for $q=\mathrm{H}$. For this purpose use will be made of $(2.26)$ (with $\mathrm{H}$ replaced by $\mathrm{H}+1$ ). In view of the equalities of $(2.16 \mathrm{~b})$ it follows that

$$
\prod_{r=1}^{i_{\alpha}} \cdots=\exp \left[\left(\delta_{1} q_{1}+\cdots+\delta_{m} q_{m}\right) \tau\right]\left[\tau, \rho_{\alpha}^{\prime}\right]
$$

where, since $\nu_{1}+\cdots+\nu_{i_{\alpha}}=\gamma_{\alpha}$ (cf. (2.26)), $\rho_{n}^{\prime}$ is given by (8.17b). Next it is inferred that

$$
\sum_{\nu_{1}, \cdots, \nu_{i \alpha}} \sum_{k_{1}^{1}, \cdots, k_{m}}^{(2)} \prod_{r=1}^{i_{\alpha}} \cdots=\text { generic form of (8.22). }
$$

Denoting the first member above by $F_{\alpha}^{\prime}$, in view of $(2.19 \mathrm{~b})$, it is deduced that

$$
\text { (8.23a) } \prod_{\alpha=1}^{n} F_{\alpha}^{\prime}=\exp \left[\left(h_{1} q_{1}+\cdots+h_{m} q_{m}\right) \tau\right]\left[\tau, \rho_{s}^{\prime \prime}\right] \quad\left(s=i_{1}+\cdots+i_{n}\right) \text {, }
$$

where, by $(8.17 \mathrm{a})$

$$
\rho_{s}^{\prime \prime}=\sum \rho_{\alpha}^{\prime}=\left(3 m^{\prime}+1\right) \sum \gamma_{\alpha}-\left(2 m^{\prime}+1\right) \sum i_{\alpha} .
$$

Now, since $\mathrm{H}$ in (2.26) has been replaced by $\mathrm{H}+1$, in consequence of (2.24) one has $\gamma_{1}+\cdots+\gamma_{n}=\mathrm{H}+1$. Thus $\rho_{s}^{\prime \prime}=\left(3 m^{\prime}+1\right)(\mathrm{H}+1)-\left(2 m^{\prime}+1\right) s$, where $s=2,3, \cdots, \mathrm{H}+1$. Whence $\rho_{s}^{\prime \prime} \leqq \rho_{2}^{\prime \prime}=\rho_{H}+m^{\prime}$ and, accordingly, 


$$
{ }_{j} a_{i_{1}}, \cdots, i_{n} \prod_{\alpha=1}^{n} F_{\alpha}^{\prime}=\exp \left[\left(h_{1} q_{1}+\cdots+h_{m} q_{m}\right) \tau\right]\left[\tau, \rho_{H}+m^{\prime}\right] .
$$

Extending over the left member of the above relation the summation symbol with the superscript (4), one finally obtains

$$
\begin{aligned}
{ }_{H+1} \Gamma_{j}^{h_{1}, \cdots, h_{m}} & =\exp \left[\left(h_{1} q_{1}+\cdots+h_{m} q_{m}\right) \tau\right]\left[\tau, \rho_{H}+m^{\prime}\right] \\
& \left(h_{1}, \cdots, h_{m} \geqq 0 ; h_{1}+\cdots+h_{m}=H+1 ; j=1, \cdots, n\right) .
\end{aligned}
$$

Thus it is seen that the relations $(8.20),(8.20 \mathrm{a})$ hold for $q=1,2, \cdots$. The following result has been established.

LEMMA 8. Consider the non-linear system (B), the associated linear system (LB) (matrix system $\left(\mathrm{LB}_{1}\right)$ ) and the matrix solution (8.4) of $\left(\mathrm{LB}_{1}\right)$ (cf. (8.3), the statement regarding the zeros of $E(\alpha)$, (8.4a), (8.4b)). With (8.6) denoting the sectors in each of which the $R\left(q_{i} t\right)(i=1, \cdots, n)$ do not change sign, let $S$ be a particular sector of the set (8.6) such that there are some $q_{i}$, say $q_{i_{1}}, q_{i_{2}}, \cdots, q_{i_{m}}(1 \leqq m \leqq n)$, all distinct from zero, for which $R\left(q_{i_{\tau}} t\right) \leqq 0$ $(\tau=1, \cdots, m ; t$ in $S)$. As a matter of notation designate the $q_{i_{\tau}} t$ involved in these inequalities as $q_{1} t, q_{2} t, \cdots, q_{m} t$. The system (B) possesses a formal solution

$$
s_{j}(t)={ }_{1} y_{j}(t)+{ }_{2} y_{j}(t)+\cdots
$$

$$
(j=1, \cdots, n)
$$

where

$$
\begin{aligned}
{ }_{\nu} y_{j}(t)= & \sum_{k_{1}, \cdots, k_{m}}{ }_{c_{1}^{k_{1}}} \cdots c_{m}^{k_{m}} \exp \left[\left(k_{1} q_{1}+\cdots+k_{m} q_{m}\right) t\right]\left[t, \rho_{\nu}\right] \\
& \left(k_{1} \geqq 0, \cdots, k_{m} \geqq 0 ; k_{1}+\cdots+k_{m}=\nu ; \nu=1,2, \cdots\right) .
\end{aligned}
$$

Here $c_{1}, \cdots, c_{m}$ are arbitrary constants, $\rho_{\nu}=\nu\left(3 m^{\prime}+1\right)-\left(2 m^{\prime}+1\right)\left(m^{\prime}\right.$ the greatest of the numbers $(8.4 \mathrm{~b})$ and $\left[t, \rho_{\nu}\right]$ represents generically a polynomial in $t$ of at most the $\rho_{\nu}$ th degree.

Note. For $\nu \geqq \nu^{\prime}$ ( $\nu^{\prime}$ sufficiently great) the $\rho_{\nu}$ can be taken smaller compared to the $\rho_{\nu}$ given in the lemma. In fact, one may take $\rho_{\nu}=\nu\left(3 m^{\prime}\right)+a^{\prime}\left(\nu \geqq \nu^{\prime}\right)$. This is because $h_{1} q_{1}+\cdots+h_{m} q_{m}-q_{\lambda}\left(h_{1} \geqq 0, \cdots, h_{m} \geqq 0 ; h_{1}+\cdots+h_{m}=\nu\right)$ will certainly be distinct from zero (for $\lambda=1, \cdots, n$ ) whenever $\nu$ is sufficiently great.

9. The Second Existence Theorem. In general, the series (8.24) will not converge in $S$ or in any given regular subregion (cf. Definition 3, \$2) of $S$. However, as seen from the Existence Theorem of $\$ 7$ there exist "actual solutions," asymptotic to the formal solutions (7.24) (in the sense of that theorem) in certain regular subregions of $S$.

We shall follow up the method previously employed in establishing the 
theorem of $\$ 7$, in order to obtain certain simplifications not directly obtainable from that theorem; more precisely, in order to obtain better results for the problem now under consideration.

The $c_{i}$ will be again subjected to the inequalities (6.2). Applying transformation (2.1) and (2.1a) to the system (B) of $\$ 8$ the inequalities (6.3) and (6.3a) should be satisfied. Now, in general, for $t$ in $S$ (6.3) will not hold, no matter how small $c^{\prime}$ is taken (unless $c_{1}=c_{2}=\cdots=c_{m}=0$, which is a case of no interest). This statement is seen to be true because of the presence of the polynomials $\left[t, \rho_{\nu}\right]$. When the roots of $E(\alpha)=0$ (cf. (8.3)) are all distinct, (6.3) can be made to hold in $S$ by taking $c^{\prime}$ sufficiently small. We have $S=S\left(\alpha_{1}, \alpha_{2} ; r\right)$ (Definition $\left.9, \S 8\right)$. There exists a regular subregion of $S$,

$$
R^{\prime}=R\left(\alpha_{1}, \alpha_{2}\right)
$$

(Definition 3, §2), such that

$$
\left|e^{q_{i} t} t^{3 m^{\prime}+1}\right| \leqq 1
$$$$
\left(i=1, \cdots, m ; t \text { in } R^{\prime}\right) . \dagger
$$

In $R^{\prime}$ (6.3) can be satisfied with $c^{\prime}(>0)$ sufficiently small. Assume, for the present, that $c^{\prime}$ has been so chosen. In place of $(6.10 \mathrm{~b})$ one now has

$$
{ }_{H} \Gamma_{j}^{\prime h_{1}, \cdots, h_{m}}(t)=\exp \left[\left(h_{1} q_{1}+\cdots+h_{m} q_{m}\right) t\right]\left[t, \rho_{H-1}+m^{\prime}\right] .
$$

Thus (6.14) will become

$$
\begin{array}{r}
-F_{j}(t)=\sum_{\nu \geqq N} \sum_{k_{1}+\cdots+k_{m}=\nu} c_{1}^{k_{1}} \cdots c_{m}^{k_{m}} \exp \left[\left(k_{1} q_{1}+\cdots\right.\right. \\
\left.\left.\quad+k_{m} q_{m}\right) t\right]\left[t, \rho_{\nu-1}+m^{\prime}\right] \\
=t^{-4 m^{\prime}-2} \sum_{\nu \geqq N} \sum_{k_{1}+\cdots+k_{m}=\nu} c_{1}^{k_{1}} \cdots c_{m}^{k_{m}} \exp \left[\left(k_{1} q_{1}+\cdots\right.\right. \\
\left.\left.\quad+k_{m} q_{m}\right) t\right] t^{\nu\left(3 m^{\prime}+1\right)}\left[\frac{1}{t}, \cdots\right],
\end{array}
$$

where the $[(1 / t), \cdots]$ are polynomials in $(1 / t)$. Hence, in view of (9.1a),

with

$$
\left|F_{j}(t)\right|<k_{0}\left(c^{\prime}\right)^{N}|t|^{-4 m^{\prime}-2} \sum_{k_{1}+\cdots+k_{m}=N} g_{k_{1}, \cdots, k_{m}}(t) \quad\left(t \text { in } R^{\prime}\right)
$$

$$
\begin{array}{r}
g_{k_{1}, \cdots, k_{m}}(t)=\left|\exp \left[\left(k_{1} q_{1}+\cdots+k_{m} q_{m}\right) t\right] t^{N\left(3 m^{\prime}+1\right)}\right| \\
\left(k_{1}+\cdots+k_{m}=N\right) .
\end{array}
$$

For $t$ in $R^{\prime}$, in place of (6.19), (6.19a), and (6.19b) one may merely assert that the

$$
\alpha_{v_{1}, \cdots, \nu_{n} ; j}(t)
$$$$
\left(\nu_{1}+\cdots+\nu_{n}=1\right)
$$

$\dagger$ The second member in (9.1a) can be replaced by any constant $(\neq \infty)$. 
and the

$$
{ }_{j} \beta_{\nu_{1}, \cdots, \nu_{n}}(t) \quad\left(\nu_{1}+\cdots+\nu_{n} \geqq 2\right)
$$

are bounded.

However the following is noted. There exists a regular subregion $R$ of $R^{\prime}$ (cf. the statement made in connection with (9.1) and (9.1a)) of the form

$$
R=R\left(\alpha_{1}, \alpha_{2}\right)
$$

(cf. Definition 3, §2) such that

$$
e^{q_{i} t} \sim 0 \quad(i=1, \cdots, m ; t \text { in } R) .
$$

Since in $R, Y_{j}(t) \sim 0(j=1, \cdots, n)$ it is observed that the functions (9.5), (9.5a) are asymptotic to zero for $t$ in $R$.

LEMMA 9. The transformation (6.1) and (6.1a) applied to the system (B) of $\$ 8$ will yield a system

$$
\rho_{j}^{(1)}(t)-l_{j}^{*}\left(t, \rho_{1}, \cdots, \rho_{n}\right)=q_{i}^{*}\left(t, \rho_{1}, \cdots, \rho_{n}\right)-F_{j}(t) \quad(j=1, \cdots, n),
$$

where

$$
\begin{array}{r}
q_{i}^{*}\left(t, \rho_{1}, \cdots, \rho_{n}\right) \sim q_{j}\left(\rho_{1}, \cdots, \rho_{n}\right), l_{j}^{*}\left(t, \rho_{1}, \cdots, \rho_{n}\right) \sim l_{j}\left(\rho_{1}, \cdots, \rho_{n}\right) \\
(j=1, \cdots, n ; t \text { in } R)
\end{array}
$$

(cf. (9.6)). $\dagger$ For $t$ in $R$ and $\left|c_{i}\right| \leqq c^{\prime}(i=1, \cdots, m)$ the $F_{j}(t)$ satisfy (9.4) and (9.4a). Also, for $t$ in $R(|t| \geqq r)$ and $\left|c_{i}\right| \leqq c^{\prime}$ the series (6.20a), representing $q_{j}^{*}$, converge absolutely and uniformly. $\ddagger$

In order to obtain a solution of the system (9.7) by methods of the type of those used in $\$ 7$, it is necessary to apply the methods of Trjitzinsky (cf. $\left(T_{2}\right)$ ) to establish the character of the solutions (within appropriate regions) of the linear system

$$
\rho_{j}^{(1)}(t)-l_{j}^{*}\left(t, \rho_{1}, \cdots, \rho_{n}\right)=0 \quad(j=1, \cdots, n) .
$$

Now (9.8) is equivalent to the matrix equation

$$
\left(\rho_{i, j}^{(1)}(t)\right)=\left(\rho_{i, j}\right)\left(l_{i, j}^{*}(t)\right) \quad(i, j=1, \cdots, n),
$$

where, by $(9.7 \mathrm{a})$,

$$
\left(l_{i, j}^{*}(t)\right) \sim\left(l_{i, j}\right)
$$

$(t$ in $R)$.

$\dagger$ In (9.7a) $q_{j}^{*} \sim q_{j}$, for instance, is to mean that in $q_{j}^{*}$ and $q_{j}$ the corresponding coefficients of the powers of $\rho_{1}, \cdots, \rho_{n}$ differ by functions of $t$, asymptotic to zero in $R$.

$\ddagger$ With proper choice of $r$ and $c^{\prime}$. 
In view of $(9.8 \mathrm{~b})$ the formal matrix solution of $(9.8 \mathrm{a})$ is the same as that of $\left(\mathrm{LB}_{1}\right)$ of $\S 8$; that is, it is given by (8.4). While the matrix (9.4) happens to constitute an "actual" matrix solution of $\left(\mathrm{LB}_{1}\right)$, in general of course it is not an "actual" solution of (9.8a). Regarding "actual" solutions, we recall the pertinent facts derivable from the Fundamental Existence Theorem of $\left(\mathrm{T}_{2}\right)$ and briefly outlined in $\$ 2$ (cf. the text in connection with $(2.6)-(2.8 \mathrm{c})$ ). To make applications to (9.8a) one needs to write

$$
S(t)=\left(e^{q_{i} t} p_{i, j}(t)\right)=\left(e^{q_{i} t} t^{r_{i}} p_{i, j}(t)\right)
$$

and let $Q_{i}(t)=q_{i} t(i=1, \cdots, n)$. Here the $r_{i}$ are certain integers and the ${ }_{1} p_{i, j}(t)$ are polynomials in $(1 / t)$. All the $B_{i, j}$ curves, that is the regular curves along which

$$
\left.R\left[\left(q_{i}-q_{j}\right) t\right]=0 \quad \text { (whenever } q_{i} \neq q_{j}\right)
$$

and the $B_{i}$ curves

$$
R\left(q_{i} t\right)=0 \quad\left(\text { whenever } q_{i} \neq 0\right),
$$

are rays of the form $\angle t=a$ constant.

Definition 11. $S^{\prime}$ is to denote any sector which can be constructed as follows. Consider the sectors separated by the rays (9.10) $(i, j=1, \cdots, n)$, no such rays extending interior to any of those sectors. There will be some sectors (of the above set) containing subsectors, interior to each of which a number of the functions $R\left(q_{i} t\right)$ is negative. $†$ Take a particular such subsector $S^{\prime \prime}$,

$$
S^{\prime \prime}=S^{\prime \prime}\left(\gamma_{1}, \gamma_{2} ; r\right) \quad\left(\gamma_{1}<\gamma_{2}\right)
$$

(cf. Definition 9, §8). As a matter of notation we may now write

$$
\begin{aligned}
& R\left(q_{1} t\right) \leqq \cdots \leqq R\left(q_{m} t\right) \leqq R\left(q_{m+1} t\right) \leqq \cdots \leqq R\left(q_{n} t\right), \\
& R\left(q_{i} t\right) \leqq 0 \quad\left(q_{i}\right.\left.\neq 0 ; i=1, \cdots, m ; t \text { in } S^{\prime}\right) .
\end{aligned}
$$

$A$ sector $S^{\prime}=S\left(\gamma_{1}+\epsilon_{1}, \gamma_{2}-\epsilon_{2} ; r\right)$ is defined as follows. If the ray $\angle t=\gamma_{1}$ (ray $\angle t=\gamma_{2}$ ) is not coincident with any of the rays

$$
R\left(q_{i} t\right)=0 \quad(i=1, \cdots, m), \quad R\left[\left(q_{i}-q_{\lambda}\right) t\right]=0 \quad(i \leqq m),
$$

take $\epsilon_{1}=0\left(\right.$ take $\left.\epsilon_{2}=0\right)$; in the contrary case we take $\epsilon_{1}$ (take $\left.\epsilon_{2}\right)$ positive, however small.

It is observed that in $S^{\prime}$ (9.11) holds and

$$
e^{q_{i} t} \sim 0
$$$$
\left(i=1, \cdots, m ; t \text { in } S^{\prime}\right)
$$

moreover,

$\dagger$ This is because, by hypothesis, not all $q_{i}$ are zero. 


$$
\left|q_{i}\right| \cos \left(\bar{q}_{i}+\bar{t}\right) \leqq-\epsilon<0
$$$$
\left(i=1, \cdots, m ; t \text { in } S^{\prime}\right) .
$$

In following the developments of $\$ 7$, as applied to the present problem, with $t$ in the sector $S^{\prime}$, it is noted that the number $\bar{m}$, introduced in connection with (7.2), can now be taken equal to $m$. The inequalities (9.13a) will correspond to (7.4a). Lemma $6, \S 4$ (with $\bar{m}=m$ ) will be valid for $t$ in $S^{\prime}\left(|t| \geqq r^{\prime}\right.$, where $r^{\prime}=r^{\prime}\left(\epsilon_{1}, \epsilon_{2}, h\right)$ ). The latter fact can be inferred by noting that the functions (7.7) and (7.7a) (for $\bar{m}=m$ ) are monotone (cf. Definition 8, $\S 7$ ) in $S^{\prime}$ (sufficiently far from the origin). This fact is deduced by observing that the statement made in connection with $(7.7 \mathrm{~b})$ holds for the problem under consideration when $t$ is in $S^{\prime}$ if we take into account the remarks made in connection with the rays $(9.12)$.

With $S^{\prime}=S\left(\delta_{1}, \delta_{2} ; r\right)\left(\delta_{1}<\delta_{2}\right)$ denoting a sector satisfying the conditions of Definition 11, in consequence of $\left(\mathrm{T}_{2}\right)$ one may assert the following.

The system $(9.8 \mathrm{a})$ possesses the matrix solutions

$$
\left(\rho_{i, j}^{\prime}(t)\right), \quad\left(\rho_{i, j}^{\prime \prime}(t)\right),
$$

with elements analytic in $S^{\prime}$ (for $t \neq \infty$ ), such that with $\epsilon>0$, however small,

$$
\begin{aligned}
& \left(\rho_{i, j}^{\prime}(t)\right) \sim\left(e^{q_{i} t} p_{i, j}(t)\right) \quad\left(t \text { in } S_{1}^{\prime}=S\left(\delta_{1}, \delta_{2}-\epsilon ; r\right)\right), \\
& \left(\rho_{i, j}^{\prime \prime}(t)\right) \sim\left(e^{q_{i} t} p_{i, j}(t)\right) \quad\left(t \text { in } S_{2}^{\prime}=S\left(\delta_{1}+\epsilon, \delta_{2} ; r\right)\right) .
\end{aligned}
$$

Here the $p_{i, j}(t)$ are the polynomials of (8.4).

The existence theorem of $\$ 7$ is applicable to the problem (B) for $t$ in $S_{1}^{\prime}$ and, also, for $t$ in $S_{2}^{\prime}$. However, with the aid of the following lemma it will be shown that an existence theorem for the problem (B) can be stated for $t$ in the more extensive region $S^{\prime}$ as well.

LEMмa 10. Let $S^{\prime}$ be a sector of the type specified in Definition 11. The system (9.8a) possesses a matrix solution $\left(\rho_{i, j}(t)\right)$, with elements analytic in $S^{\prime}$ $(t \neq \infty)$, such that on writing

$$
\begin{aligned}
\left(\rho_{i, j}(t)\right) & =\left(e^{q_{i} t} t^{m^{\prime}} b_{i, j}(t)\right), \\
\left(\rho_{i, j}(t)\right)^{-1} & =\left(\bar{\rho}_{i, j}(t)\right)=\left(t^{m^{\prime}} \bar{b}_{i, j}(t) e^{-q_{j} t}\right)
\end{aligned}
$$

$\left(m^{\prime}=\right.$ greatest of numbers $\left.(9.4 \mathrm{~b})\right)$ we have

$$
\left|b_{i, j}(t)\right|<\gamma, \quad\left|\bar{b}_{i, j}(t)\right|<\gamma \quad\left(i, j=1, \cdots, n ; t \text { in } S^{\prime}\right) .
$$

To prove this we note that the matrix solutions involved in (9.15), (9.15a) are connected by the relation

$$
\left(\rho_{i, j}^{\prime}(t)\right)=\left(c_{i, j}\right)\left(\rho_{i, j}^{\prime \prime}(t)\right)=\left(\sum_{\lambda} c_{i, \lambda} \rho_{\lambda, j}^{\prime \prime}(t)\right)
$$


where the $c_{i, j}$ are certain constants. Let $o(t)$ generically denote a function which in the indicated region is asymptotic to zero. By (9.15a) and (8.5a) we have

$$
\left(\rho_{i, j}^{\prime \prime}(t)\right)^{-1}=\left(\bar{\rho}_{i, j}^{\prime \prime}(t)\right)=\left(\left(\bar{p}_{i, j}(t)+o(t)\right) e^{-q_{j} t}\right)
$$

$\left(\right.$ in $\left.S_{2}^{\prime}\right)$.

From (9.17) and (9.15), in view of (9.17a),

$$
\begin{array}{r}
\left(c_{i, j}\right)=\left(\rho_{i, j}^{\prime}(t)\right)\left(\bar{\rho}_{i, j}^{\prime \prime}(t)\right)=\left(e^{\left(q_{i}-q_{j}\right) t}\left[\sum_{\lambda} p_{i, \lambda}(t) p_{\lambda, j}+o(t)\right]\right) \\
\left(\text { in } S_{3}^{\prime}=S\left(\delta_{1}+\epsilon, \delta_{2}-\epsilon ; r\right)\right) .
\end{array}
$$

By (8.5b)

$$
\left(c_{i, j}\right)=\left(e^{\left(q_{i}-q_{j}\right) t}\left[\delta_{i, j}+o(t)\right]\right)
$$

Letting $t \rightarrow \infty$ in $S_{3}^{\prime}$ we infer that $c_{i, i}=1(i=1, \cdots, n)$. Moreover, by (9.11) $c_{i, j} \rightarrow 0(i<j)$ when $|t| \rightarrow \infty$ in $S_{3}^{\prime}$; that is,

$$
c_{i, j}=0
$$

Thus, by (9.17) and (9.15a),

$$
\begin{aligned}
\rho_{i, j}^{\prime}(t) & =\rho_{i, j}^{\prime \prime}(t)+\sum_{\lambda=1}^{i-1} c_{i, \lambda} \rho_{\lambda, j}^{\prime \prime}(t) \\
& =e^{q_{i} t}\left[p_{i, j}(t)+o(t)+\sum_{\lambda=1}^{i-1} c_{i, \lambda} e^{\left(q_{\lambda}-q_{i}\right) t}\left(p_{\lambda, j}(t)+o(t)\right)\right] \quad\left(\text { in } S_{2}^{\prime}\right) .
\end{aligned}
$$

In consequence of (9.11)

$$
\left|e^{\left(q_{\lambda}-q_{i}\right) t}\right| \leqq 1 \quad\left(\lambda=1, \cdots, i-1 ; t \text { in } S_{2}^{\prime}\right) ;
$$

moreover, the $p_{i, j}(t)(i, j=1, \cdots, n)$ are of the form $\left[t, m^{\prime}\right]$. Hence the $\rho_{i, j}^{\prime}(t)$ are of the form (9.16), (9.16b) for $t$ in $S_{2}^{\prime}$. By virtue of (9.15) it is seen that they are of the form (9.16), (9.16b) in $S^{\prime}$ as well. With $\epsilon$ sufficiently small $S^{\prime}=S_{1}^{\prime}+S_{2}^{\prime}$. Accordingly, for $t$ in $S^{\prime}$, the matrix $\left(\rho_{i, j}^{\prime}(t)\right)$ will satisfy the conditions (9.16), (9.16b) of the lemma.

By (9.15), (9.15a), and (8.5a)

$$
\begin{array}{ll}
\left(\rho_{i, j}^{\prime}(t)\right)^{-1}=\left(\bar{\rho}_{i, j}(t)\right)=\left(\left(\bar{p}_{i, j}(t)+o(t)\right) e^{-q_{i} t}\right) & \text { (in } \left.S_{1}^{\prime}\right) \\
\left(\rho_{i, j}^{\prime \prime}(t)\right)^{-1}=\left(\bar{\rho}_{i, j}^{\prime \prime}(t)\right)=\left(\left(\bar{p}_{i, j}(t)+o(t)\right) e^{-q_{j} t}\right) & \text { (in } \left.S_{2}^{\prime}\right) .
\end{array}
$$

If we write

$$
\left(c_{i, j}\right)^{-1}=\left(\bar{c}_{i, j}\right)
$$

it follows that 


$$
\bar{c}_{i, j}+\sum_{\lambda=j+1}^{n} \bar{c}_{i, \lambda} c_{\lambda, j}=\delta_{i, j}
$$

From (9.21) (for $j=n$ ) we deduce that $\bar{c}_{n, n}=1$ and $\bar{c}_{i, n}=0(i<n)$. For $j=n-1$ (9.21) will yield $\bar{c}_{n-1, n-1}=1, \bar{c}_{i, n-1}=0(i<n-1)$; and so on. Thus

$$
\bar{c}_{i, i}=1 \quad(i=1, \cdots, n),
$$$$
\bar{c}_{i, j}=0
$$

By (9.17)

$$
\left(\bar{\rho}_{i, j}^{\prime}(t)\right)=\left(\bar{\rho}_{i, j}^{\prime \prime}(t)\right)\left(\bar{c}_{i, j}\right)=\left(\sum_{\lambda} \bar{\rho}_{i, \lambda}^{\prime \prime}(t) \bar{c}_{\lambda, j}\right) .
$$

In view of $(9.21 \mathrm{a})$ and $(9.20 \mathrm{a})$

$$
\begin{aligned}
\bar{\rho}_{i, j}^{\prime}(t) & =\bar{\rho}_{i, j}^{\prime \prime}+\sum_{\lambda=j+1}^{n}\left(\bar{p}_{i, \lambda}(t)+o(t)\right) e^{-q_{\lambda} t \bar{c}_{\lambda, j}} \\
& =e^{-q_{j} t}\left[\bar{p}_{i, j}(t)+o(t)+\sum_{\lambda=j+1}^{n}\left(\bar{p}_{i, \lambda}(t)+o(t)\right) \exp \left[\left(q_{j}-q_{\lambda}\right) t\right] \bar{c}_{\lambda, j}\right],
\end{aligned}
$$

for $t$ in $S_{2}^{\prime}$. Here, by (9.11),

$$
\left|\exp \left[\left(q_{j}-q_{\lambda}\right) t\right]\right| \leqq 1 \quad\left(\lambda=j+1, \cdots, n ; t \text { in } S_{2}^{\prime}\right) .
$$

Now, as we have noted before, the $\bar{p}_{i, j}(t)$ are of the form $\left[t, m^{\prime}\right]$. Hence, in consequence of (9.22a), (9.22b), it is observed that

$$
\left|\bar{\rho}_{i, j}^{\prime}(t)\right|<\gamma^{\prime}|t|^{m^{\prime}} e^{-q_{j} t} \quad\left(i, j=1, \cdots, n ; t \text { in } S_{2}^{\prime}\right) .
$$

By (9.20) the $\left|\bar{\rho}_{i, j}(t)\right|$ satisfy inequalities of the same type in $S_{1}^{\prime}$. Hence, with a suitable $\gamma^{\prime}$ (9.23) will hold in $S^{\prime}$. This completes the proof of the lemma. $\dagger$

A solution of the system (9.7) will be obtained in the form of a convergent series (7.22), the terms of this series being defined by the relations (7.19)(7.21b) (with $p=0$ ). In place of (7.23) we now have

$$
w_{j: k}(t)=\sum_{\lambda=1}^{n} c_{\lambda}^{j, k}(t) \rho_{\lambda, j}(t)
$$

where

$$
c_{\lambda}^{i, k}(t)=\sum_{\lambda_{1}=1}^{n} \int^{t} g_{\lambda_{1}: k}(\tau) \bar{\rho}_{\lambda_{1}, \lambda}(\tau) d \tau
$$

(cf. $(2.28)-(2.30 \mathrm{~b}))$. Here the $\rho_{i, j}(t)$ and the $\bar{\rho}_{i, j}(\tau)$ are the functions referred to in Lemma 10. With the aid of (9.16), (9.16a), (9.16b) one obtains from (9.24), (9.24a), provided $\left|g_{j: k}\right| \leqq g_{k}$,

$\dagger$ Either of the matrices $\left(\rho_{i, j}^{\prime}\right),\left(\rho_{i, j}^{\prime \prime}\right)$ can be taken for the matrix $\left(\rho_{i, j}\right)$ of the lemma. 


$$
\left|w_{j: k}(t)\right|<n \gamma^{2} \sum_{\lambda=1}^{n}\left|e^{q_{\lambda} t t^{m^{\prime}}}\right| \int^{t}\left|g_{k}\right|\left|e^{-q_{\lambda} \tau^{m^{\prime}+1+\epsilon^{\prime}}}\right|\left|\tau^{-1-\epsilon^{\prime}} d \tau\right|
$$

for $t$ in $S^{\prime}$ (provided that the involved integrals converge).

It is essential to note that, for $t$ in $S^{\prime}$, the inequalities (9.25) are of the same type as those of (7.24) and that Lemma $6, \S 7$, as we remarked subsequent to $(9.13 \mathrm{a})$, is applicable. Repeating the processes of $\$ 7$, integrating along rays $\angle \tau=\angle t\left(|\tau| \geqq|t| ; t\right.$ in $\left.S^{\prime}\right)$, the following theorem is now deduced.

The Second Existence Theorem. Consider the system (B), §8. ( $\left.\mathrm{LB}_{1}\right), \S 8$ will represent a related linear system. Let the $q_{i}$ denote the roots of the characteristic equation (9.3) (a root of multiplicity $m_{j}$ repeated $m_{j}$ times). It is assumed that not all the $q_{i}$ are zero. Let $S^{\prime}$ denote a sector satisfying the conditions of Definition 11. Thus, as a matter of notation, in $S^{\prime}$ we have (9.11), (9.13), (9.13a). Let $N$ be any integer $\geqq \nu(\epsilon)$; here, with $\epsilon$ denoting the number in the second members of $(9.13 \mathrm{a}), \nu(\epsilon)$ is the least integer equal to or greater than $\left(\epsilon+\left|q_{\lambda}\right|\right) / \epsilon$ $(\lambda=1, \cdots, m-1)$.

The system (B), $\$ 8$ possesses a solution, $y_{j}(t)(j=1, \cdots, n)$, whose elements are analytic in $S^{\prime}(t \neq \infty)$. At $t=\infty$ they will be singular. Moreover,

$$
y_{j}(t) \sim s_{j}(t) \quad\left(j=1, \cdots, n ; t \text { in } S^{\prime}\left(|t| \geqq r^{*}\right)\right)
$$

where the $s_{j}(t)$ are the formal series (8.24) (cf. (8.24a)) of Lemma 8, §8. The asymptotic relations $(9.26)$ are to be interpreted as follows. The $y_{j}(t)$ are functions of the form

$$
\text { (9.26a) } \begin{aligned}
y_{j}(t)= & \sum_{\nu=1}^{N-1} \sum_{k_{1}+\cdots+k_{m}=\nu} c_{1}^{k_{1}} \cdots c_{m}^{k_{m}} \exp \left[\left(k_{1} q_{1}+\cdots\right.\right. \\
& \left.\left.+k_{m} q_{m}\right) t\right]_{\nu} p_{k_{1}, \cdots, k_{m} ; j}(t)+\rho_{j}(t)
\end{aligned}
$$

where $c_{1}, \cdots, c_{m}$ are arbitrary constants $\left(\left|c_{i}\right| \leqq c^{\prime} ; i=1, \cdots, m\right)$ and the ${ }_{\nu} p_{k_{1}}, \ldots, k_{m} ;(t)$ are polynomials in $t$ of at most the $\left[\nu\left(3 m^{\prime}+1\right)-\left(2 m^{\prime}+1\right)\right]$ st degree. $\dagger$ The $\rho_{j}(t)=\rho_{j}\left(c_{1}, \cdots, c_{m} ; t\right)$ are analytic, for $t \neq \infty$, in $S^{\prime}\left(|t| \geqq r^{*}\right)$ and satisfy in this sector the inequalities

(9.26b) $\left|\rho_{j}(t)\right|<h^{\prime}\left(c^{\prime}\right)^{N}|t|^{N m^{\prime \prime}} \sum_{k_{1}+\cdots+k_{m}=N}\left|\exp \left[\left(k_{1} q_{1}+\cdots+k_{m} q_{m}\right) t\right]\right|$,

provided $\left|c_{i}\right| \leqq c^{\prime}(i=1, \cdots, m)$; here $m^{\prime \prime}$ is a constant independent of $N$. In the above we have assumed that $c^{\prime}$ is sufficiently small and $r^{*}$ is sufficiently great.

Note. In this theorem the term "asymptotic" is justified for reasons of the same type as stated in the note subsequent to the theorem of $\S 7$.

10. Uniqueness theorem. Turning our attention to the theorem of $\$ 7$ we observe that there is a manifold of solutions, depending on $\bar{m}$ parameters $c_{i}$,

$\dagger m^{\prime}$ is the greatest of the numbers $(8.4 \mathrm{~b})$. 
such that the elements of the solutions, as well as all the derivatives of these elements, approach zero as $|t| \rightarrow \infty$ within $W_{\xi}$. This set of solutions has been constructed corresponding to some $N(\geqq \nu(\xi))$. We shall write

$$
y_{j}(t) \underset{N}{\sim} s_{j}(t) \quad\left(j=1, \cdots, n ; t \text { in } W_{\xi}\left(|t| \geqq r_{N}\right) ;\left|c_{i}\right| \leqq c^{\prime}\right),
$$

where $\widetilde{N}$ is to signify (7.50a) and (7.50c); this symbol will be read as "asymptotic to $N$ terms." It appears at the first glance that the $y_{j}(t)$ may depend on $N$.

Let the $y_{j}^{\prime}(t)$ be elements of a solution of (A), $\$ 1$ such that

$$
y_{j}^{\prime}(t) \underset{N+1}{{ }^{\prime}} s_{j}(t) \quad\left(j=1, \cdots, n ; t \text { in } W_{\xi}\left(|t| \geqq r_{N+1}\right) ;\left|c_{i}\right| \leqq c^{\prime}\right) .
$$

We may have $r_{N+1} \geqq r_{N}$. Form the difference

$$
z_{j}(t)=y_{j}^{\prime}(t)-y_{j}(t)={ }_{N} y_{j}(t)+\rho_{j}^{\prime}(t)-\rho_{j}(t) ;
$$

here ${ }_{N} y_{j}(t)$ is given by (5.23a) and (5.24a), and $\rho_{j}^{\prime}(t)$ are the $\rho_{j}(t)$ of the theorem of $\S 7$, formed with $N$ replaced by $N+1$. If we take account of $(7.50 \mathrm{c}$ ) and of the note of $\S 7$, from (10.3) we infer that

(10.3a) $\quad\left|z_{j}(t)\right|<h^{*}{ }_{N} \rho^{*}(t)+{ }_{N+1} \rho^{*}(t) \quad\left(t\right.$ in $\left.W_{\xi} ;|t| \geqq r_{N+1} ;\left|c_{i}\right| \leqq c^{\prime}\right) . \dagger$

By (10.3a) and (7.50c)

$$
\begin{aligned}
& \quad \begin{array}{l}
z_{j}(t) \mid<\zeta \cdot N \rho^{*}(t) \\
=\zeta \zeta^{\prime}\left(c^{\prime}\right)^{N}|t|^{(N-1) \bar{\omega}+\xi+2 \epsilon^{\prime}} \sum_{k_{1}+\cdots+k_{\bar{m}}=N} \mid \exp \left[k_{1} Q_{1}(t)+\cdots\right. \\
\left.\quad+k_{\bar{m}} Q_{\bar{m}}(t)\right] t^{k_{1} r_{1}+\cdots+k_{\bar{m}} r_{\bar{m}}} \mid=z_{1}^{*}(t) \quad\left(t \text { in } W_{\xi}\left(|t| \geqq r_{N+1}\right)\right) ;
\end{array}
\end{aligned}
$$

also, by the theorem of $\$ 7$,

$$
\left|y_{j}(t)\right|<\eta_{1} \rho^{*}(t)|t|^{-\xi-2 \epsilon^{\prime}}=y^{*}(t) \quad\left(t \text { in } W_{\xi}\left(|t| \geqq r_{N+1}\right)\right) .
$$

It will be assumed that $r_{N+1}$ has been taken sufficiently great so that

$$
z_{1}^{*}(t)+y^{*}(t) \leqq \rho^{*}<\rho \quad\left(t \text { in } W_{\xi} ;|t| \geqq r_{N+1}\right)
$$

(cf. (1.2)).

By (10.4), (10.4a), (10.4b), in consequence of the statement in connection with (7.17), $\ddagger$ we have

$$
\begin{aligned}
\mid q_{j}\left(t, y_{1}+z_{1}, \cdots, y_{n}+z_{n}\right)-q_{j}\left(t, y_{1},\right. & \left.\cdots, y_{n}\right) \mid \\
& <\left(q^{\prime} y^{*}(t)+q z_{1}^{*}(t)\right) z_{1}^{*}(t)
\end{aligned}
$$

$\dagger$ Take $r_{N+1} \geqq r_{N}$.

$\ddagger$ One now has $q_{j}$ in place of $q_{j}^{*}$; however $q_{j}$ is of the same description as $q_{j}^{*}$. 
for the indicated values of the variables. Hence

$$
\begin{array}{r}
\left|q_{j}\left(t, y_{1}+z_{1}, \cdots\right)-q_{j}\left(t, y_{1}, \cdots\right)\right|<a^{\prime}{ }_{1} \rho^{*}(t)_{N} \rho^{*}(t)|t|-\xi-2 \mathbf{e}^{\prime} \\
\left(t \text { in } W_{\xi} ;|t| \geqq r_{N+1}\right) .
\end{array}
$$

Subtracting the corresponding members of the relations

$$
\begin{aligned}
& t^{-p}{y_{j}^{\prime}}^{(1)}(t)-l_{j}\left(t, y_{1}^{\prime}, \cdots, y_{n}^{\prime}\right)=q_{j}\left(t, y_{1}^{\prime}, \cdots, y_{n}^{\prime}\right), \\
& t^{-p} y_{j}(t)-l_{j}\left(t, y_{1}, \cdots, y_{n}\right)=q_{j}\left(t, y_{1}, \cdots, y_{n}\right) \\
& \quad(j=1, \cdots, n)
\end{aligned}
$$

(cf. (A), $\$ 1,(1.1 \mathrm{a}),(1.1 \mathrm{~b}))$, we find that in consequence of $(10.3)$

$$
z_{j}^{(1)}(t)-t^{p} l_{j}\left(t, z_{1}(t), \cdots, z_{n}(t)\right)=t^{p} T_{j}(t) \quad(j=1, \cdots, n),
$$

where

$$
T_{j}(t)=q_{j}\left(t, y_{1}+z_{1}, \cdots\right)-q_{j}\left(t, y_{1}, \cdots\right) .
$$

Thus, by (10.5),

$$
\left|T_{j}(t)\right|<a^{\prime}{ }_{1} \rho^{*}(t)_{N} \rho^{*}(t)|t|-\xi-2 \epsilon^{\prime} \quad\left(t \text { in } W_{\xi} ;|t| \geqq r_{N+1}\right) . \dagger
$$

It is noted that the left members in (10.7) are of the form

$$
L_{j}\left(t, z_{1}(t), \cdots, z_{n}(t)\right),
$$

where the differential operator $L_{j}$ is precisely the same as the one so denoted in $\left(S_{H}\right), \S 2$ (also cf. $(2.14 \mathrm{a})$ ). If we note that $\left(S_{H}\right), \S 2$ can be solved with the aid of (2.30) and (2.30a) and that these formulas may be written in the form (5.4), (5.4a), and (5.4b), the relations (10.7) may be written as follows:

$$
z_{j}(t)=\sum_{\lambda=1}^{n} c_{\lambda}(t) e^{Q_{\lambda}(t)} t^{r} \gamma_{\lambda, j}(t)
$$

where

$$
\begin{aligned}
c_{\lambda}(t) & =\sum_{\lambda_{1}=1}^{n} \int^{t} T_{\lambda_{1}}(\tau) e^{-Q_{\lambda}(\tau)} \tau^{-r_{\lambda}+\omega+p} \bar{\gamma}_{\lambda_{1}, \lambda}(\tau) d \tau, \\
\gamma_{\lambda, j}(\tau) & =[\tau]^{*}, \quad \bar{\gamma}_{\lambda_{1}, \lambda}(\tau)=[\tau]^{*} \quad\left(\tau \text { in } W_{\xi} ;|t| \geqq r_{N+1}\right) .
\end{aligned}
$$

The formulas (10.8), (10.8a), with the integrals taken as indefinite, would give a general solution of $(10.7)$ if $z_{1}(t), \cdots, z_{n}(t)$ were considered as the unknowns and the $T_{j}(t)$ were considered as known functions of $t$. In the case on hand, however, the $z_{j}(t)$ by definition are known functions. Hence (10.8),

$\dagger$ Throughout the discussion we keep $\left|c_{i}\right| \leqq c^{\prime}(i=1, \cdots, \bar{m})$. 
(10.8a) will be equivalent to (10.7) only if the integrations in (10.8a) are suitably specified. One may write

$$
c_{\lambda}(t)=d_{j, \lambda}+\sum_{\lambda_{1}=1}^{n} \int_{\infty}^{t} T_{\lambda_{1}}(\tau) e^{-Q_{\lambda}(\tau)} \tau^{-r_{\lambda}+\omega+p} \bar{\gamma}_{\lambda_{1}, \lambda}(\tau) d \tau,
$$

where the path of integration (for $t$ in $W_{\xi}$ ) is within $W_{\xi}$ and the $d_{j, \lambda}$ are certain constants. The integrals here involved exist in view of (10.7b). The integrand displayed in (10.9) being analytic in $W_{\xi}(t \neq \infty)$, the path of integration will be chosen as the ray

$$
\angle \tau=\angle t \quad\left(|\tau| \geqq|t| ; t \text { in } W_{\xi}\right) .
$$

Substituting (10.9) in (10.8) one has

$$
z_{j}(t)=\zeta_{j}(t)+\zeta_{j}^{\prime}(t),
$$

where

$$
\zeta_{j}^{\prime}(t)=\sum_{\lambda=1}^{n} d_{j, \lambda} e^{Q_{\lambda}(t)} t^{r} \gamma_{\lambda, j}(t) \quad(j=1, \cdots, n) ;
$$

on the other hand the $\zeta_{j}(t)$ will satisfy inequalities, $†$ provided $\left|T_{\lambda_{1}}(\tau)\right|<T(\tau)$, $(10.10 \mathrm{~b})\left|\zeta_{j}(t)\right|<n \gamma_{0}{ }^{2} \sum_{\lambda=1}^{n}\left|e^{Q_{\lambda}(t)} t^{r_{\lambda}}+\epsilon^{\prime}\right| \int_{\infty}^{t}\left|T(\tau) e^{-Q_{\lambda}(\tau)} \tau^{-r_{\lambda}+\bar{\omega}+2 \epsilon^{\prime}}\right|\left|\tau^{-1-\epsilon^{\prime}} d \tau\right|$.

In consequence of $(10.7 \mathrm{~b})$ and in view of the notation introduced at the beginning of $\$ 7$ (cf. (7.1)-(7.6))

$$
\begin{aligned}
& \left|T(\tau) e^{-Q_{\lambda}(\tau)} \tau^{-r_{\lambda}+\bar{\omega}+2 \epsilon^{\prime}}\right|<a^{\prime}{ }_{1} \rho^{*}(\tau)_{N} \rho^{*}(\tau)|\tau|^{-\xi-2 \epsilon^{\prime}}\left|e^{-Q_{\lambda}(\tau)} \tau^{-r_{\lambda}+\bar{\omega}+2 \epsilon^{\prime}}\right|
\end{aligned}
$$

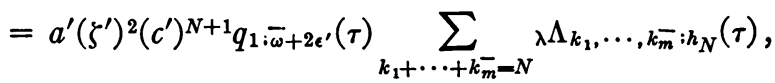

where $h_{N}=\bar{\omega}+\left(\xi+2 \epsilon^{\prime}\right) /(N-1)$. Hence, by Lemma $6, \S 7$, the second member of (10.11) is monotone (cf. Definition $8, \S 7$ ) in

$$
W_{\xi}
$$

$$
\left(|t| \geqq r^{\prime}(\xi, h) \geqq r_{N+1}\right),
$$

where $h$ is the greater one of the numbers $\bar{\omega}+2 \epsilon^{\prime}$ and $h_{N}$. Hence the integral displayed in $(10.10 \mathrm{~b})$ is less than the second member of (10.11) (with $\tau=t$ ) multiplied by $|t|^{-\epsilon^{\prime}} / \epsilon^{\prime}$, provided $t$ is in the region (10.11a). Thus, by (10.10b),

$$
\begin{aligned}
& \left|\zeta_{j}(t)\right|<\frac{a^{\prime} n^{2} \gamma_{0}^{2}}{\epsilon^{\prime}}|t|^{\bar{\omega}-\xi_{1} \rho^{*}(t)} \rho_{N} \rho^{*}(t)=z_{2}^{*}(t)=g(t) z_{1}^{*}(t),
\end{aligned}
$$

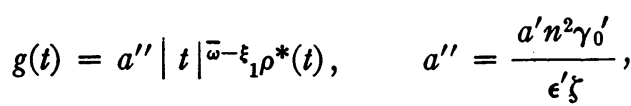
as in $\$ 7$. 
for $t$ in the region (10.11a). In (10.11a) take $r^{\prime}(\xi, h)$ also sufficiently great so that in the indicated region

$$
g(t) \leqq \delta<1 .
$$

Since, except for a constant factor, $g(t)$ is of the form

$$
|t|^{\bar{\omega}+2 \epsilon^{\prime}} q_{1: 0}(t)
$$

(cf. (7.1a)), by virtue of the definition of $h$ it follows that $g(t)$ is monotone in the region (10.11a).

If we make use of (10.10), (10.4), (10.12), and (10.12b) we conclude that

$$
\left|\zeta_{j}^{\prime}(t)\right|<(1+\delta) z_{1}^{*}(t) \quad\left(t \text { in } W_{\xi} ;|t| \geqq r^{\prime}(\xi, h)\right) .
$$

Let $R$ be some fixed ray interior to $W_{\xi}$. On $R$

$$
\begin{aligned}
R Q_{1}(t)=\cdots=R Q_{n_{1}}(t)<R Q_{n_{1}+1}(t)=\cdots=R Q_{n_{2}}(t)<\cdots & \\
<R Q_{n_{\sigma-1}+1}(t)=\cdots=R Q_{n_{-1}}(t)<R Q_{n_{\sigma-1}+1}(t)=\cdots= & R Q_{n_{\sigma}}(t) \\
& \quad\left(n_{\sigma}=n\right)
\end{aligned}
$$

and

$$
\exp \left[Q_{i}(t)-Q_{j}(t)\right] \sim 0
$$

for $i<j$, whenever $Q_{i}(t) \not \equiv Q_{j}(t)$. In view of (10.10a) one may write

$$
\zeta_{j}^{\prime}(t)=e^{Q_{n_{\sigma}}(t)} \zeta_{j, \sigma}^{\prime}(t)+e^{Q_{n_{\sigma}-1}(t)} \zeta_{j, \sigma-1}^{\prime}(t)+\cdots+e^{Q_{n_{1}}(t)} \zeta_{j, 1}^{\prime}(t),
$$

where

$$
\text { (10.15a) } \quad \zeta_{j, \nu}^{\prime}(t)=\sum_{\lambda=n_{\nu-1+1}}^{n_{\nu}} d_{j, \lambda} t^{r_{\lambda}} \gamma_{\lambda, j}(t)
$$

$$
\left(n_{0}=0 ; \nu=1, \cdots, \sigma\right)
$$

With $o(t)$ denoting generically a function $\sim 0$ (for the indicated values of $t$ ), in consequence of (10.14a), (10.15), and (10.15a) we have

$$
\zeta_{j}^{\prime}(t)=e^{Q_{n_{\sigma}}(t)}\left[\zeta_{j, \sigma}^{\prime}(t)+o(t)\right]
$$

$(t$ on $R)$.

By virtue of (10.13)

$$
\left|\zeta_{j, \sigma}^{\prime}(t)+o(t)\right|<(1+\delta) z_{1}^{*}(t) e^{-Q_{n \sigma}(t)}
$$

$(t$ on $R)$.

If note is taken of the properties of the functions (7.6), for $k_{1}+\cdots+k_{\bar{m}}=\nu$ $\geqq N \geqq \nu(\xi)$, it follows that the second member in (10.16a) is $\sim 0$ along $R$. Hence the function of (10.15a) for $\nu=\sigma$ must be $\sim 0$ for $t$ on $R$. In view, however, of the origin of the functions involved in the second member of $(10.15 \mathrm{a})$ for $\nu=\sigma$ this would imply that necessarily

$$
\zeta_{j, \sigma}^{\prime}(t) \equiv 0
$$$$
(j=1, \cdots, n) .
$$ 
We substitute $(10.16 \mathrm{~b})$ in $(10.15)$ and prove that $\zeta_{i, \sigma-1}^{\prime}(t) \equiv 0(j=1, \cdots, n)$ in a way analogous to that used in establishing (10.16b). Thus, step by step it is shown that the $\zeta_{j, v}^{\prime}(t)$ are all identically zero. Hence, from (10.10) and (10.12) it follows that

$$
\left|z_{j}(t)\right|<g(t) z_{1}^{*}(t)=z_{2}^{*}(t) \quad(j=1, \cdots, n)
$$

in the region (10.11a) (where $r^{\prime}(\xi, h)$ is assumed to satisfy the condition stated in connection with $(10.12 b))$.

We repeat the procedure beginning with (10.4), replacing (10.4) by (10.17), and letting tremain in the region (10.11a). It is observed that $(10.4 \mathrm{~b})$ will be satisfied, with $z_{1}^{*}(t)$ replaced by $z_{2}^{*}(t)$, since $g(t)<1$. Moreover, $(10.4 \mathrm{c})$ with $z_{1}^{*}(t)$ replaced by $z_{2}^{*}(t)$ will hold. Now

$$
\left(q^{\prime} y^{*}(t)+q z_{2}^{*}(t)\right) z_{2}^{*}(t)<\left[\left(q^{\prime} y^{*}(t)+q z_{1}^{*}(t)\right) z_{1}^{*}(t)\right] g(t),
$$

so that (10.5) becomes

$$
\left(10.5^{\prime}\right) \quad\left|q_{j}\left(t, y_{1}+z_{1}, \cdots\right)-q_{j}\left(t, y_{1}, \cdots\right)\right|<a_{1}^{\prime} \rho^{*}(t)_{N} \rho^{*}(t)|t|^{-\xi-2 \epsilon^{\prime}} g(t) \text {. }
$$

Whence in the second member of $(10.7 \mathrm{~b})$ we may introduce $g(t)$ as a factor. The argument is the same until we obtain the relation (10.11). The second and the third members of (10.11) are to be multiplied by $g(t)$. Designate the resulting inequalities by $(10 . \overline{11})$. Now, as remarked before, $g(\tau)$ is monotone in the region (10.11a) (with $r^{\prime}(\xi, h)$ chosen so that (10.12b) holds). Along the path of integration indicated in $(10.10 \mathrm{~b}) g(\tau) \leqq g(t)$. Hence in place of (10.12) one now obtains

$$
\left|\zeta_{j}(t)\right|<g^{2}(t) z_{1}^{*}(t)=z_{3}^{*}(t) \quad(t \text { in the region (10.11a)). }
$$

As established before, we have $\zeta_{j}^{\prime}(t) \equiv 0$. Thus in (10.18) $\zeta_{j}(t)$ may be replaced by $z_{j}(t)$.

It is not difficult to prove by induction that

$$
\left|z_{j}(t)\right|<g^{k}(t) z_{1}^{*}(t) \quad(j=1, \cdots, n ; k=1,2, \cdots)
$$

for $t$ in the region (10.11a) (such that (10.12b) holds). The latter region is independent of $k$. Let $t=t_{0}$ be any point in the above region; since by $(10.12 \mathrm{~b})$ and (10.19)

$$
\left|z_{j}\left(t_{0}\right)\right|<\delta^{k} z_{1}^{*}\left(t_{0}\right) \quad(j=1, \cdots, n ; k=1,2, \cdots),
$$

where the second member can be made as small as desired by a suitable choice of $k$, it follows that $z_{j}\left(t_{0}\right)=0(j=1, \cdots, n)$. Thus

$$
z_{j}(t) \equiv 0 \quad(j=1, \cdots, n) .
$$

Whence, by (10.3), it is concluded that the solutions referred to in (10.1) and 
(10.2) are identical. It is thus inferred that the elements of the solution described in the First Existence Theorem are independent of $N$ so long as $N \geqq \nu(\xi)$. The same result will hold for the theorem of $\$ 9$, even though the latter theorem is not a special case of the first. One needs only to apply the result established for the theorem of $\$ 7$ to a suitable subsector (a ray, even, is sufficient) of the sector for which the theorem of $\$ 9$ has been stated; (10.20) will then hold in the original sector in consequence of a well known property of analytic functions.

UNIQUENESS THEOREM. The solutions of the existence theorems of $\$ \$ 7$ and 9 are independent of $N$. Thus, if

$$
y_{1}(t), y_{2}(t), \cdots, y_{n}(t)
$$

are the elements of a solution of the non-linear differential system under consideration, and if

$$
s_{1}(t), s_{2}(t), \cdots, s_{n}(t)
$$

are the corresponding elements of a formal solution, we have

$$
y_{j}(t) \underset{N}{\sim} s_{j}(t) \quad(j=1, \cdots, n ; t \text { in the indicated region }),
$$

where $N=\nu(\xi), \nu(\xi)+1, \nu(\xi)+2, \cdots$. For any fixed $N(\geqq \nu(\xi))$ the "asymptotic" relations (10.21) are to signify that the $y_{j}(t)$ are functions possessing the properties described in the existence theorems referred to above.

Note. The above theorem, then, implies that the solutions mentioned in the theorems of $\$ \$ 7$ and 9 are asymptotic to the corresponding formal series not merely "to $N$ terms," as stated in those theorems, but also in the ordinary sense; that is, to infinitely many terms.

11. Systems (C). Our purpose will now be to investigate in the complex neighborhood of the singular point $\lambda=\infty$ the analytic character of some of the solutions of systems (C), mentioned in $\$ 1$. A system (C) will be assumed to be of the following form:

(11.1) $\lambda^{-p} y_{j}^{(1)}(x)=a_{j}\left(\lambda, x, y_{1}, \cdots, y_{n}\right) \quad(j=1, \cdots, n ; p$ an integer $\geqq 0), \dagger$ where $\lambda$ is a parameter and

$$
\begin{aligned}
\text { (11.1a) } & a_{j}\left(\lambda, x, y_{1}, \cdots, y_{n}\right)=l_{j}\left(\lambda, x, y_{1}, \cdots, y_{n}\right)+q_{j}\left(\lambda, x, y_{1}, \cdots, y_{n}\right), \\
(11.1 \mathrm{~b}) & l_{j}\left(\lambda, x, y_{1}, \cdots, y_{n}\right)=l_{1, j}(\lambda, x) y_{1}+\cdots+l_{n, j}(\lambda, x) y_{n}, \\
& \quad q_{j}\left(\lambda, x, y_{1}, \cdots, y_{n}\right)=\sum{ }_{j} a_{i_{1}}, \cdots, i_{n}(\lambda, x) y_{1}^{i_{1}} \cdots y_{n} \\
(11.1 \mathrm{c}) & \left(i_{1}, \cdots, i_{n} \geqq 0 ; i_{1}+\cdots+i_{n} \geqq 2 ; j=1, \cdots, n\right) .
\end{aligned}
$$

$\dagger$ Indicated derivatives are with respect to $x$. 
Here the $l_{i, j}(\lambda, x),{ }_{j} a_{i_{1}}, \ldots, i_{n}(\lambda, x)$ are functions representable by series of the form

$$
c_{0}(x)+c_{1}(x) \lambda^{-1}+c_{2}(x) \lambda^{-2}+\cdots,
$$

where the $c_{\nu}(x)(\nu=0,1, \cdots)$ are continuous, together with their derivatives of all orders, for $x$ on the closed interval $(\alpha, \beta)$. Moreover, the series (11.2) are assumed to converge absolutely and uniformly $\dagger$ for $x$ in $\left(\alpha_{1}, \beta_{1}\right)$ and for

$$
|\lambda| \geqq r .
$$

The series involved in the second member of (11.1c) are assumed to be absolutely and uniformly convergent $\ddagger$ for

$$
\alpha_{1} \leqq x \leqq \beta_{1} ;|\lambda| \geqq r ;\left|y_{1}\right|,\left|y_{2}\right|, \cdots,\left|y_{n}\right| \leqq \rho .
$$

Moreover, it will be assumed that the system obtained by replacing the $q_{j}$ by zeros is actually of order $n$.

Associated with the problem (11.1) there is a linear system

$$
\lambda^{-p} y_{j}^{(1)}(x)=l_{j}\left(\lambda, x, y_{1}, \cdots, y_{n}\right) \quad(j=1, \cdots, n) .
$$

A general analytic theory for systems of this type has been given by Trjitzinsky. It will be assumed that the reader is familiar with the results and methods contained in $\left(\mathrm{T}_{3}\right)$. In connection with the earlier contributions to the theory of linear systems containing a parameter, we shall mention papers of outstanding importance by G. D. Birkhoff, $\|$ J. D. Tamarkin $\uparrow$ and P. Noaillon. $\dagger \dagger$ Some additional references to the contributions of these and some other writers may be found in $\left(\mathrm{T}_{3}\right)$.

$\dagger$ The functions under consideration will be analytic in $\lambda$ for $|\lambda| \geqq r(\lambda=\infty$ included) when $x$ has any fixed value in $\left(\alpha_{1}, \beta_{1}\right)$, if it is assumed (as it is) that $r$ is sufficiently great. For every fixed $\lambda$ $(|\lambda| \geqq r)$ these functions are continuous in $x$ for $x$ in $\left(\alpha_{1}, \beta_{1}\right)$, in consequence of the assumed uniformity of convergence of the series (11.2). This continuity is uniform with respect to $\lambda(|\lambda| \geqq r)$.

$\ddagger r$ is taken sufficiently great and $\rho$ sufficiently small so that, for $x$ in $\left(\alpha_{1}, \beta_{1}\right)$, the functions $q_{j}\left(\lambda, x, y_{1}, \cdots, y_{n}\right)$ are analytic in $\left(\lambda, y_{1}, \cdots, y_{n}\right)$, at $\left(\lambda=\infty, y_{1}=y_{2}=\cdots=y_{n}=0\right)$, for $(|\lambda| \geqq r$; $\left.\left|y_{1}\right|,\left|y_{2}\right|, \cdots,\left|y_{n}\right| \leqq \rho\right)$. For any set of values, such that (11.4) holds, the $q_{j}$ will be continuous in $x$ for $x$ in $\left(\alpha_{1}, \beta_{1}\right)$.

$\S$ W. J. Trjitzinsky, Theory of linear differential equations containing a parameter, Acta Mathematica, vol. 67 (1936), pp. 1-50. In the sequel this paper will be referred to as $\left(\mathrm{T}_{3}\right)$.

|| G. D. Birkhoff, On the asymptotic character of solutions of certain linear differential equations, these Transactions, vol. 9 (1908), pp. 219-231.

I J. D. Tamarkin, Some general problems of the theory of linear differential equations and expansions . . . , Mathematische Zeitschrift, vol. 27 (1927), pp. 1-54.

†† P. Noaillon, Développements asymptotiques dans les équations différentielles linéaires à paramètre variable, Mémoires de la Société des Sciences de Liege, (3), vol. 9 (1912), 197 pp. 
12. Preliminaries for systems (C). The linear system (11.5) is equivalent to the matrix equation

$$
\begin{aligned}
Y^{(1)}(x) & =Y(x) A(\lambda, x), & & Y(x)=\left(y_{i, j}(x)\right), \\
A(\lambda, x) & =\left(\lambda^{p} l_{i, j}(\lambda, x)\right) & & (i, j=1, \cdots, n)
\end{aligned}
$$

(cf. (11.1b)). A solution of (12.1) will consist of the $n$ elements in a row of $Y(t)$. Now, this system is of the type to which the results of $\left(\mathrm{T}_{3}\right)$ apply. It is to be noted that the linear system on hand possesses a singularity at $\lambda=\infty$.

It will be convenient to introduce the following definition.

Definition 1. Generically $\{\lambda, x\}$ (integer $q \geqq 0$ ) is to denote a series possibly divergent for all $\lambda \neq \infty$, of the form

$$
\sigma_{0}(x)+\sigma_{1}(x) \lambda^{-1 / k}+\cdots+\sigma_{\nu}(x) \lambda^{-\nu / k}+\cdots
$$

(integer $k>0)$. Here $\sigma_{\nu}(x)(\nu=0,1, \cdots)$ is, together with the derivatives of all orders (which are assumed to exist), continuous on some real interval.

The system (12.1) possesses a formal matrix solution $\dagger$

$$
S(\lambda, x)=\left(s_{i, j}(\lambda, x)\right)=\left(e^{Q_{i}(\lambda, x)} \sigma_{i, j}(\lambda, x)\right) .
$$

$$
\begin{aligned}
Q_{i}(\lambda, x) & =\sum_{\nu=0}^{l_{i}-1} q_{\nu}{ }^{i}(x) \lambda^{\left(l_{i}-\nu\right) / k_{i}} & & \text { (integers } \left.k_{i} \geqq 1\right), \\
\sigma_{i, j}(\lambda, x) & =\{\lambda, x\} & & (i, j=1, \cdots, n)
\end{aligned}
$$

(cf. Definition 1), the functions of $x$ in the second members of (12.2a) and (12.2) being, together with the derivatives of all orders, continuous on a subinterval $\left(\alpha_{2}, \beta_{2}\right)$ of $\left(\alpha_{1}, \beta_{1}\right) . \ddagger$ Henceforth, unless stated otherwise, attention will be confined to a particular such interval $\left(\alpha_{2}, \beta_{2}\right)$ and its subintervals. The interval $\left(\alpha_{2}, \beta_{2}\right)$ is taken so that for no $x$ in $\left(\alpha_{2}, \beta_{2}\right)$ do any two roots of the characteristic equation of (12.1) coincide, unless these two roots coincide throughout $\left(\alpha_{2}, \beta_{2}\right)$.§ The intervals under consideration are closed.

By the Fundamental Existence Theorem of $\left(\mathrm{T}_{3}\right)$ in particular the following can be asserted.

Let $\left(\alpha_{2}, \beta_{2}\right)$ be an interval as stated above. Let the $B_{i, j}^{1, x}$ designate curves in the $\lambda$-plane, extending to infinity, along which

$\dagger$ That is, the determinant $|S(x, \lambda)|$ does not formally reduce to zero.

$\ddagger$ It is to be noted that $\left(\alpha_{1}, \beta_{1}\right)$ may contain values of $x=x_{0}$, such that in two intervals contained in $\left(\alpha_{1}, \beta_{1}\right)$ on different sides of $x_{0}$ the system (12.1) will possess distinct formal matrix solutions (12.2).

$\S$ That is, $\left(\alpha_{2}, \beta_{2}\right)$ is such that roots which are distinct for some $x$ in $\left(\alpha_{2}, \beta_{2}\right)$ remain distinct throughout $\left(\alpha_{2}, \beta_{2}\right)$; while any two roots coincident for some $x$ in $\left(\alpha_{2}, \beta_{2}\right)$ will coincide throughout this interval. 


$$
R Q_{i}^{(1)}(\lambda, x)=R Q_{j}^{(1)}(\lambda, x) . \dagger
$$

There exist subintervals of $\left(\alpha_{2}, \beta_{2}\right)$ such that, when $x$ is restricted to one of them, there exists a regular (cf. Definition $3, \S 2)$ region $R$ in the $\lambda$-plane so that there are no curves $B_{i, j}^{1, x}$ interior to $R$. Let $\left(\alpha^{\prime}, \beta^{\prime}\right)$ denote any particular subinterval mentioned above. As a matter of notation we may write

$$
\begin{array}{r}
R Q_{1}^{(1)}(\lambda, x) \leqq R Q_{2}^{(1)}(\lambda, x) \leqq \cdots \leqq R Q_{n}^{(1)}(\lambda, x) \\
\quad\left(x \text { in }\left(\alpha^{\prime}, \beta^{\prime}\right) ; \lambda \text { in } R\right) .
\end{array}
$$

Let $\alpha$ be an integer, however large. The system (12.1) possesses a matrix solution ${ }_{\alpha} Y(\lambda, x)$, with elements analytic in $\lambda(\lambda \neq \infty)$ and continuous in $x$ for $x$ in $\left(\alpha^{\prime}, \beta^{\prime}\right)$ and $\lambda$ in $R$; moreover, for the indicated values of the variables $\ddagger$

$$
{ }_{\alpha} Y(\lambda, x) \underset{\alpha}{\sim} S(\lambda, x)
$$

(cf. (12.2)).

Consider a matrix solution satisfying (12.5). Form another matrix solution

$$
Y(\lambda, x)=\left(y_{i, j}(\lambda, x)\right)=\left(\delta_{i, j} e^{-Q_{j}\left(\lambda, \alpha^{\prime}\right)}\right)_{\alpha} Y(\lambda, x),
$$

where $\left(\delta_{i, j}\right)$ is the identity matrix. We shall have

$$
Y(x, \lambda) \underset{\alpha}{\sim} S^{*}(\lambda, x)=\left(e^{G i(\lambda, x)} \sigma_{i, j}(\lambda, x)\right) \quad\left(x \text { in }\left(\alpha^{\prime}, \beta^{\prime}\right) ; \lambda \text { in } R\right)
$$

(cf. $(12.2 \mathrm{~b}))$, and

$$
G_{i}(\lambda, x)=Q_{i}(\lambda, x)-Q_{i}\left(\lambda, \alpha^{\prime}\right)
$$

Since, $\S$ by $(12.6 b)$,

and

$$
G_{i}(\lambda, x)=\int_{u=\alpha^{\prime}}^{x} G_{i}{ }^{(1)}(\lambda, u) d u
$$

$$
R G_{i}(\lambda, x)=\int_{u=\alpha^{\prime}}^{x} R Q_{i}^{(1)}(\lambda, u) d u
$$

$\dagger$ Whenever $Q_{i}(\lambda, x) \equiv Q_{j}(\lambda, x)$, the corresponding $B_{i, j}^{1, x}$ curves will be said not to exist. Throughout it will be understood that these curves extend to infinity and that sufficiently far from the origin they are simple; moreover, at infinity they will possess limiting directions. A particular curve (12.3) will depend on $x$; as $x$ varies in the interval this curve may vary, remaining always interior to a sector; the angle of this sector can be made as small as desired by taking the interval for $x$ suitably small (cf. $\left.\left(\mathrm{T}_{3}\right)\right)$.

$\ddagger \sim$ is to denote an asymptotic relation in the ordinary sense (unless the contrary is stated); that is, to infinitely many terms. $\widetilde{\alpha}$ denotes an asymptotic relation, to $\alpha$ terms. These relations are with respect to $\lambda$ and are "uniform" with respect to $x\left(x\right.$ in $\left.\left(\alpha^{\prime}, \beta^{\prime}\right)\right)\left(\right.$ (cf. $\left.\left(T_{3}\right)\right)$.

$\S$ Throughout, differentiation is never with respect to $\lambda$. 
if it is noted that

$$
R\left(G_{\nu}(\lambda, x)-G_{k}(\lambda, x)\right)=\int_{u=\alpha^{\prime}}^{x} R\left(Q_{\nu}^{(1)}(\lambda, u)-Q_{k}^{(1)}(\lambda, u)\right) d u
$$

in view of (12.4) it is concluded that

$$
R G_{\nu}(\lambda, x) \leqq R G_{k}(\lambda, x),
$$

whenever $\nu<k, x$ is in $\left(\alpha^{\prime}, \beta^{\prime}\right)$ and $\lambda$ is in $R$. Thus, for the indicated values of the variables

$$
R G_{1}(\lambda, x) \leqq R G_{2}(\lambda, x) \leqq \cdots \leqq R G_{n}(\lambda, x) ;
$$

moreover, if for $x$ in $\left(\alpha^{\prime}, \beta^{\prime}\right)$ and $\lambda$ in $R$ and some $i$, we have $R Q_{i}^{(1)}(\lambda, x) \leqq 0$, it will follow that for the same values of the variables

$$
R G_{i}(\lambda, x) \leqq 0 .
$$

The case of interest is when not all the $Q_{i}^{(1)}(\lambda, x)$ are identically zero. This will be assumed.

Definition 2. $\Gamma\left(\alpha^{\prime}, \beta^{\prime} ; R\right)$ will denote the aggregate of the values of $x$ and $\lambda$ such that

$$
\alpha^{\prime} \leqq x \leqq \beta^{\prime} \text { and } \lambda \text { is in } R
$$

$\left(\alpha^{\prime}<\beta^{\prime} ; R\right.$ a regular region (cf. Definition $\left.\left.3, \S 2\right)\right) \Gamma\left(\alpha^{\prime}, \beta^{\prime} ; R\right)$ will be said to be proper if

(i) The interval $\left(\alpha^{\prime}, \beta^{\prime}\right)$ possesses the properties attributed to $\left(\alpha_{2}, \beta_{2}\right)$ in the italicized statement subsequent to (12.2b).

(ii) With suitable notation, we may assert the validity of (12.4) in $\Gamma\left(\alpha^{\prime}, \beta^{\prime} ; R\right)$.

It is observed that if we take the interval for $x$ sufficiently small, proper regions $\Gamma\left(\alpha^{\prime}, \beta^{\prime} ; R\right)$ can always be constructed. By the Fundamental Existence Theorem of $\left(\mathrm{T}_{3}\right)$ it follows that, given any particular proper region $\Gamma\left(\alpha^{\prime}, \beta^{\prime} ; R\right)$ and any $\alpha$, however large, there exists a matrix solution of (12.1), say ${ }_{\alpha} Y(\lambda, x)$, which satisfies in $\Gamma\left(\alpha^{\prime}, \beta^{\prime} ; R\right)$ the conditions of the italicized statement made in connection with (12.5).

Since not all the $R Q_{i}{ }^{(1)}(\lambda, x)$ are identically zero, in view of the statement in connection with (12.7a), it is inferred that there exist proper regions with the additional property

$$
R Q_{1}^{(1)}(\lambda, x) \leqq \cdots \leqq R Q_{m}^{(1)}(\lambda, x) \leqq 0 ;
$$

that is,

$$
R G_{1}(\lambda, x) \leqq \cdots \leqq R G_{m}(\lambda, x) \leqq 0,
$$


satisfied in the region under consideration $(1 \leqq m \leqq n)$.

Hence it is not difficult to see that there exist regions satisfying the following definition.

Definition 3. A region $\Gamma\left(c, d ; R^{\prime}\right)$ (notation of Definition 2) will be said to be admissible if it has the following properties:

(a) There exists a region $\Gamma\left(\alpha^{\prime}, \beta^{\prime} ; R\right)$, proper according to Definition 2 , such that the interval $(c, d)$ is contained in $\left(\alpha^{\prime}, \beta^{\prime}\right)$ and $R^{\prime}$ is contained in $R$. With suitable notation we may then write

$$
R Q_{1}{ }^{(1)}(\lambda, x) \leqq R Q_{2}{ }^{(1)}(\lambda, x) \leqq \cdots \leqq R Q_{n}{ }^{(1)}(\lambda, x)
$$

in $\Gamma\left(\alpha^{\prime}, \beta^{\prime} ; R\right)$ (we may also write (12.7); cf. (12.6b)).

(b) For some $m(1 \leqq m \leqq n)$

$$
R Q_{i}^{(1)}(\lambda, x) \leqq 0
$$

$$
\left(i=1, \cdots, m ; \text { in } \Gamma\left(c, d ; R^{\prime}\right)\right)
$$

It is observed that (12.10a) implies

$$
R G_{i}(\lambda, x) \leqq 0
$$

$$
\left(i=1, \cdots, m ; \text { in } \Gamma\left(c, d ; R^{\prime}\right)\right) .
$$

(c) Whenever the number $m$ employed in (b) exceeds unity we also have

$$
R Q_{1}{ }^{(1)}(\lambda, x) \geqq 2 R Q_{m}^{(1)}(\lambda, x)
$$

Admissible regions for which $m=1$ (that is, (a) and (b) are satisfied and (c) is deleted) can be always constructed. Admissible regions with $m>1$ will exist in a wide variety of problems.

Consider now a particular admissible region $\Gamma\left(c, d ; R^{\prime}\right)$, subset of a proper region $\Gamma\left(\alpha^{\prime}, \beta^{\prime} ; R\right)$. Let

$$
Y(\lambda, x)=\left({ }_{0} y_{i, j}(\lambda, x)\right)
$$

be a matrix solution of (12.1) satisfying (12.6a) (cf. (12.6b)) and (12.10) in $\Gamma\left(\alpha^{\prime}, \beta^{\prime} ; R\right)$; moreover, in $\Gamma\left(c, d ; R^{\prime}\right)$ we shall have $(12.10 \mathrm{a}),(12.10 \mathrm{~b}),(12.10 \mathrm{c}) . \dagger$

On turning to the system (11.5) it is noted that a solution can be taken in the form

$$
{ }_{1} y_{j}(\lambda, x)=\sum_{\sigma=1}^{m} c_{\sigma} 0 y_{\sigma, j}(\lambda, x) \quad(j=1, \cdots, n),
$$

where $c_{1}, c_{2}, \cdots, c_{m}$ are arbitrary constants (which may depend on $\lambda$ ). We shall seek to satisfy the non-linear system (11.1) with the aid of the formal series

$$
\begin{aligned}
y_{j}(\lambda, x)={ }_{1} y_{j}(\lambda, x)+{ }_{2} y_{j}(\lambda, x)+\cdots+{ }_{\nu} y_{j}(\lambda, x)+ & \cdots \\
& (j=1, \cdots, n),
\end{aligned}
$$

$\dagger(12.10 \mathrm{c})$ is deleted if $m=1$. 
where ${ }_{1} y_{j}(\lambda, x)$ is given by (12.12). The ${ }_{\imath} y_{j}(\lambda, x)$ will be determined as functions of the form

$$
\begin{aligned}
& { }_{\nu} y_{j}(\lambda, x)=\sum_{k_{1}, \cdots, k_{m}} c_{1}^{k_{1}} \cdots c_{m}^{k_{m}}{ }_{\nu} \eta_{k_{1}, \cdots, k_{m} ; j}(\lambda, x) \\
& \left(k_{1}, \cdots, k_{m} \geqq 0 ; k_{1}+\cdots+k_{m}=\nu\right) .
\end{aligned}
$$

For $\nu=1$ we may write

$$
{ }_{0} y_{\sigma, j}(\lambda, x)={ }_{1} \eta_{k_{1}, \cdots, k_{m} ; j}(\lambda, x),
$$

where

$$
k_{i}=0 \quad(\text { for } i \neq \sigma), \quad k_{\sigma}=1 .
$$

Substitution of (12.13) in (11.1) will result in a set of differential relations; the coefficients involved in the second members of (12.13a) must satisfy these relations. Formulas of this kind have been calculated in $\$ 2$. It will be convenient to collect together, with some slight modifications, those of the formulas which will be needed in the sequel. We have

$$
\begin{aligned}
& L_{j}\left(\lambda, x,{ }_{H} \eta_{h_{1}}, \cdots, h_{m} ; 1, \cdots,{ }_{H} \eta_{h_{1}}, \cdots, h_{m} ; n\right)=\lambda_{H}^{p} \Gamma_{j}^{h_{1}, \cdots, h_{m}} \\
& \left(j=1, \cdots, n ; \boldsymbol{H}=h_{1}+\cdots+h_{n} ; h_{0} \geqq 0, \cdots, h_{m} \geqq 0 ; H=2,3, \cdots\right),
\end{aligned}
$$

where

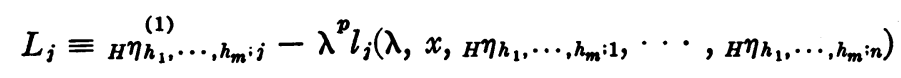

(cf. (11.1b)) and

$$
\begin{array}{r}
{ }_{H} \Gamma_{j}^{h_{1}, \cdots, h_{m}}=\sum_{j}^{(4)} a_{i_{1}, \cdots, i_{n}}(\lambda, x) \prod_{\alpha=1}^{n} \sum_{\nu_{1}+\cdots+v_{i_{\alpha}}=\gamma_{\alpha}} \sum_{k_{1}{ }^{1}, \cdots, k_{m}{ }^{i_{\alpha}}}^{(2)} \prod_{r=1}^{i_{\alpha}}{ }_{\nu_{r}} \eta_{k_{1}^{r}, \cdots, k_{m}^{r} ; \alpha} \\
\left(h_{1}+\cdots+h_{m}={ }_{H} ; h_{1} \geqq 0, \cdots, h_{m} \geqq 0\right) .
\end{array}
$$

In (12.15)

$$
\sum^{(4)}=\sum_{s=2}^{H} \sum_{i_{1}+\cdots+i_{n}=s} \sum_{\gamma_{1}+\cdots+\gamma_{n}=H} \sum_{1_{1} \delta_{1}, \cdots, j_{m}}^{(3)}\left(\gamma_{1} \geqq i_{1}, \cdots, \gamma_{n} \geqq i_{n}\right)
$$

with

$$
\begin{aligned}
& \sum^{(3)}=\sum\left[{ }_{1} \delta_{q}+\cdots+{ }_{n} \delta_{q}=h_{q}, \quad(q=1, \cdots, m) ;\right. \\
& \left.{ }_{1} \delta_{1}+\cdots+{ }_{1} \delta_{m}=\gamma_{1} ; \cdots ;{ }_{n} \delta_{1}+\cdots+{ }_{n} \delta_{m}=\gamma_{n}\right] \text {; }
\end{aligned}
$$

moreover, 


$$
\begin{array}{r}
\sum^{(2)}=\sum\left[k_{1}{ }^{1}+\cdots+k_{1}^{i_{\alpha}}=\delta_{1} ; k_{2}{ }^{1}+\cdots+k_{2}^{i_{\alpha}}=\delta_{2} ; \cdots\right. \\
k_{m}{ }^{1}+\cdots+k_{m}^{i_{\alpha}}=\delta_{m} ; k_{1}{ }^{1}+\cdots+k_{m}{ }^{1}=\nu_{1} ; \cdots ; \\
\left.k_{1}^{i_{\alpha}}+\cdots+k_{m}^{i_{\alpha}}=\nu_{i_{\alpha}}\right] .
\end{array}
$$

Also, formally,

$$
y_{j}^{(1)}(x)-\lambda^{p} a_{j}\left(\lambda, x, y_{1}, \cdots, y_{n}\right)=\sum_{H=2}^{\infty} \sum_{h_{1}+\cdots+h_{m}=H} c_{1}^{h_{1}} \cdots c_{m H}^{h_{m}} \psi_{h_{1}}, \cdots, h_{m} ; j,
$$

where

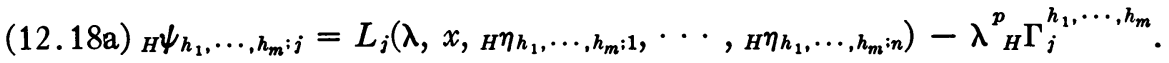

Consider a non-homogeneous system corresponding to (11.5),

$$
y_{j}^{(1)}(x)-\lambda^{p} l_{j}\left(\lambda, x, y_{1}, \cdots, y_{n}\right)=g_{j} \quad(j=1, \cdots, n) .
$$

We then have

$$
y_{j}(\lambda, x)=\sum_{\beta=1}^{n} c_{\beta}(\lambda, x)_{0} y_{\beta, j}(\lambda, x) \quad(j=1, \cdots, n)
$$

where

$$
c_{\beta}(\lambda, x)=\sum_{\tau=1}^{n} \int^{x} g_{\tau}(u)_{0} \bar{y}_{\tau, \beta}(\lambda, u) d u,
$$

provided that the integrals here involved converge. Here the ${ }_{0} y_{\sigma, j}(\lambda, x)$ are from the matrix solution (12.11), and the ${ }_{0} \bar{y}_{\tau, \sigma}(\lambda, u)$ are the elements of the inverse of the matrix $Y(\lambda, u)$; that is,

$$
\left({ }_{0} \bar{y}_{i, j}(\lambda, u)\right)=\left({ }_{0} y_{i, j}(\lambda, u)\right)^{-1} \text {. }
$$

An application of (12.19)-(12.20b) to (12.14) yields

$$
\begin{aligned}
{ }_{H} \eta_{h_{1}, \cdots, h_{m} ; j}(\lambda, x) & =\sum_{\beta=1}^{n}{ }_{H} c_{\beta}(\lambda, x)_{0} y_{\beta, j}(\lambda, x), \\
{ }_{H} c_{\beta}(\lambda, x) & =\sum_{\tau=1}^{n} \int \lambda^{x}{ }_{H} \Gamma_{\tau}^{h_{1}, \cdots, h_{m}}(u)_{0} \bar{y}_{\tau, \beta}(\lambda, u) d u .
\end{aligned}
$$

13. Formal solutions for systems (C). We shall proceed now with $\Gamma\left(\alpha^{\prime}, \beta^{\prime} ; R\right), \Gamma\left(c, d ; R^{\prime}\right)$ and $Y(\lambda, x)$ having the meaning assigned to these symbols in the italicized statement made in connection with (12.11). Thus

$$
{ }_{0} y_{\beta, j}(\lambda, x)=e^{G \beta(\lambda, x)} \gamma_{\beta, j}(\lambda, x),
$$

where 


$$
\gamma_{\beta, j}(\lambda, x) \approx\{\lambda, x\}
$$

(cf. Definition 1, \$12) in $\Gamma\left(\alpha^{\prime}, \beta^{\prime} ; R\right)$. Definition 1 here is applied, of course, for the interval $\left(\alpha^{\prime}, \beta^{\prime}\right)$. Taking account of $(12.20 \mathrm{~b})$ and noting (formally) that $\left|S^{*}(\lambda, u)\right| \not \equiv 0$ (cf. (12.6a)), we have

$$
\begin{aligned}
& { }_{0} \bar{y}_{\tau, \beta}(\lambda, u)=e^{-G_{\beta}(\lambda, u)} \lambda^{\omega} \bar{\gamma}_{\tau, \beta}(\lambda, u), \\
& \bar{\gamma}_{\tau, \beta}(\lambda, u) \underset{\widetilde{\alpha}}{\widetilde{\alpha}}\{\lambda, u\} \quad(\omega \text { an integer } \geqq 0) \text {, }
\end{aligned}
$$

where $\bar{\alpha} \leqq \alpha$ and $\bar{\alpha} \rightarrow \infty$ with $\alpha$. Also

$$
\left.\left|\gamma_{\beta, j}(\lambda, x)\right|, \quad\left|\bar{\gamma}_{\tau, \beta}(\lambda, x)\right|<\gamma \quad \text { (in } \Gamma\left(\alpha^{\prime}, \beta^{\prime} ; R\right)\right) .
$$

In place of (12.21) and (12.21a) we now may write

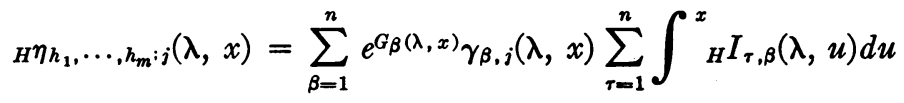

(cf. (13.1a)),

$$
{ }_{H} I_{\tau, \beta}(\lambda, u)={ }_{H} \Gamma_{\tau}^{h_{1}, \cdots, h_{m}}(u) e^{-G_{\beta}(\lambda, u)} \lambda^{p+\omega} \bar{\gamma}_{\tau, \beta}(\lambda, u)
$$

$\left(j=1, \cdots, n ;{ }_{B}=h_{1}+\cdots+h_{m} ; h_{1} \geqq 0, \cdots, h_{m} \geqq 0 ; \quad B=2,3, \cdots\right)$

(cf. (13.2a)).

For $\theta=2$ (12.15) becomes

$$
\begin{aligned}
& { }_{2} \Gamma_{j}^{h_{1}, \cdots, h_{m}}(u) \\
& \quad=\sum_{j}^{(4)}{ }_{j} a_{i_{1}, \cdots, i_{n}}(\lambda, u) \prod_{\alpha=1}^{n} \sum_{\nu_{1}+\cdots+\nu_{i_{\alpha}}=i_{\alpha}} \sum^{(2)} \prod_{r=1}^{i_{\alpha}}{ }_{\nu_{r}} \eta_{k_{1}^{r}, \cdots, k_{m}^{r} ; \alpha}(\lambda, u) .
\end{aligned}
$$

On the other hand, in view of $(12.13 \mathrm{~b})$,

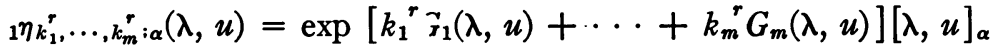

$$
\begin{aligned}
& \left(k_{1}{ }^{r}+\cdots+k_{m}{ }^{r}=1 ; \alpha=1, \cdots, n ; \lambda, u \text { in } \Gamma\left(\alpha^{\prime}, \beta^{\prime} ; R\right)\right) \text {. }
\end{aligned}
$$

Here and in the sequel $[\lambda, u]_{\alpha}$ is defined as follows.

Definition 4. Generically $[\lambda, x]_{\alpha}(\lambda, x$ in a region $\Gamma(a, b ; R)$; cf. Definition $2, \S 12)$ is to denote a function defined and such that

$$
[\lambda, x]_{\alpha} \sim\{\lambda, x\}
$$

(cf. Definition 1, §2) for $\lambda, x$ in the above region. $\dagger$

$\dagger$ That is, $[\lambda, x]_{\alpha}=\sigma_{0}(x)+\sigma_{1}(x) \lambda^{-1 / k}+\cdots+\sigma_{\alpha-1}(x) \lambda^{-(\alpha-1) / k}+\sigma_{\alpha}(x, \lambda) \lambda^{-\alpha / k}$, where $\left|\sigma_{\alpha}(x, \lambda)\right|<b$ in $\Gamma(a, b ; R)$. 
Generically $[\lambda, x]_{\alpha}[\lambda, x]_{\alpha}=[\lambda, x]_{\alpha}$ and $[\lambda, x]_{\alpha}+[\lambda, x]_{\alpha}=[\lambda, x]_{\alpha}$. In consequence of (12.17) from (13.6) we obtain

$$
\prod_{r=1}^{i_{\alpha}}{ }_{{ }_{r}} \eta_{k_{1}}^{r}, \cdots, k_{m}^{r} ; \alpha(\lambda, u)=\exp \left[\delta_{1} G_{1}(\lambda, u)+\cdots+\delta_{m} G_{m}(\lambda, u)\right][\lambda, u]_{\alpha} .
$$

Designate the expression displayed in (13.5) subsequent to the first product symbol as $F_{\alpha}^{\prime}$. In view of (13.7)

(13.7a) $\quad F_{\alpha}^{\prime}=$ generic form of (13.7)

$\left(\right.$ with $\left.\delta_{i}={ }_{\alpha} \delta_{i}\right)$.

Thus

$$
\begin{aligned}
& \prod_{\alpha=1}^{n} F_{\alpha}=\exp \left[\left({ }_{1} \delta_{1}+\cdots+{ }_{n} \delta_{1}\right)\right. G_{1}(\lambda, u) \\
&+\cdots \\
&\left.+\left({ }_{1} \delta_{m}+\cdots+{ }_{n} \delta_{m}\right) G_{m}(\lambda, u)\right][\lambda, u]_{\alpha}
\end{aligned}
$$

and, by (12.16) and (12.16a),

$(13.7 \mathrm{c}) \prod_{\alpha=1}^{n} F_{\alpha}^{\prime}=\exp \left[h_{1} G_{1}(\lambda, u)+\cdots+h_{m} G_{m}(\lambda, u)\right][\lambda, u]_{\alpha}$.

Now, we may write

$$
{ }_{j} a_{i_{1}}, \cdots, i_{n}(\lambda, u)=[\lambda, u]_{\infty}
$$

(cf. (11.2)). Also we observe that $[\lambda, u]_{\alpha}[\lambda, u]_{\infty}=[\lambda, u]_{\alpha}$. Hence

$$
{ }_{j} a_{i_{1}}, \cdots, i_{n}(\lambda, u) \prod_{\alpha=1}^{n} F_{\alpha}{ }^{\prime}=\text { generic form of }(13.7 \mathrm{c}) \text {. }
$$

It is noted, next, that in (13.5)

$$
\sum^{(4)}=\sum_{i_{1}+\cdots+i_{n}=2} \sum_{\gamma_{1}+\cdots+\gamma_{n}=2} \sum_{1_{1}, \cdots, n}^{(3)} \quad\left(\gamma_{1}=\imath_{1}, \cdots, \gamma_{n}=i_{n}\right) .
$$

Whence, by (13.5) and (13.8),

$$
{ }_{2} \Gamma_{i}^{h_{1}, \cdots, h_{m}}(u)=\exp \left[h_{1} G_{1}(\lambda, u)+\cdots+h_{m} G_{m}(\lambda, u)\right][\lambda, u]_{\alpha}
$$

for $\lambda, u$ in $\Gamma\left(\alpha^{\prime}, \beta^{\prime} ; R\right)$. Thus, in view of (13.4a) (for $\left.z=2\right)$ and (13.2a),

$$
\begin{array}{r}
{ }_{2} I_{\tau, \beta}(\lambda, u)=\exp \left[h_{1} G_{1}(\lambda, u)+\cdots+h_{m} G_{m}(\lambda, u)-G_{\beta}(\lambda, u)\right] \lambda^{p+\omega}[\lambda, u]_{\bar{\alpha}} \\
\left(h_{1}, \cdots, h_{m} \geqq 0 ; h_{1}+\cdots+h_{m}=2\right)
\end{array}
$$

when $\lambda, u$ is in $\Gamma\left(\alpha^{\prime}, \beta^{\prime} ; R\right)$.

The following lemma will now be proved.

Lemma 1. Let $\Gamma\left(c, d ; R^{\prime}\right)$ denote an admissible region (cf. Definition 3, $\$ 12)$. Then, with suitable notation, we have in it (12.10), (12.10a), and (12.10c). 
Let

$$
\begin{aligned}
Q(\lambda, x)= & h_{1} Q_{1}(\lambda, x)+h_{2} Q_{2}(\lambda, x)+\cdots+h_{m} Q_{m}(\lambda, x)-Q_{\beta}(\lambda, x) \\
& \left(h_{1}, \cdots, h_{m} \geqq 0 ; h_{1}+\cdots+h_{m}=\nu \geqq 2 ; \text { the } h_{i} \text { integers }\right) .
\end{aligned}
$$

Assume, moreover, that in $Q^{(1)}(\lambda, x)$ (unless $\left.Q^{(1)}(\lambda, x) \equiv 0\right)$ the coefficient of the highest power of $\lambda$ does not vanish in the interval (closed) $(a, b)$. We then can evaluate the integral below so that, with $w \geqq l+1$, we have

$$
\int^{x} e^{Q(\lambda, u)}[\lambda, u]_{w} d u=e^{Q(\lambda, x)}[\lambda, x]_{w-1}
$$

(cf. Definition 4), provided that the displayed integrand is of the stated form in $\Gamma\left(c, d ; R^{\prime}\right)$. Formula (13.12) will be valid for $\lambda, x$ in $\Gamma\left(c, d ; R^{\prime}\right)$. Furthermore, if the highest power of $\lambda$ in $Q^{(1)}(\lambda, x)$ is the $(l / k)$ th $\left(l / k>0\right.$; i.e., $\left.Q^{(1)}(\lambda, x) \neq 0\right)$ the coefficients of $\lambda^{-\nu / k}(\nu=0, \cdots, l-1)$ in the second member of (13.12) will all be zero.

Note. If we assume that this lemma is true, it will follow that the facts stated therein will continue to hold when the $Q_{i}(\lambda, x)$ are replaced by the $G_{i}(\lambda, x)\left(=Q_{i}(\lambda, x)-Q_{i}\left(\lambda, \alpha^{\prime}\right)\right)(i=1, \cdots, n)$, respectively.

The truth of the above lemma would follow from the developments of $\$ 4$ of $\left(T_{3}\right)$ if it were shown that

$$
R Q^{(1)}(\lambda, x) \leqq 0 \quad\left(\lambda, x \text { in } \Gamma\left(c, d ; R^{\prime}\right)\right) .
$$

Suppose $\beta>m$. Some $h_{i}$ will be positive. We then have

$$
\begin{aligned}
Q^{(1)}(\lambda, x)=\left[h_{1} Q_{1}^{(1)}(\lambda, x)\right. & +\cdots+\left(h_{i}-1\right) Q_{i}^{(1)}(\lambda, x)+\cdots \\
& \left.+h_{m} Q_{m}^{(1)}(\lambda, x)\right]+\left[Q_{i}^{(1)}(\lambda, x)-Q_{\beta}^{(1)}(\lambda, x)\right]
\end{aligned}
$$

and (13.13) will follow in consequence of (12.10a) and (12.10). $\dagger$

Consider now the case when $\beta \leqq m$. If $h_{\beta}>0$ and if we write

$$
\begin{aligned}
Q^{(1)}(\lambda, x)=h_{1} Q_{1}^{(1)}(\lambda, x)+\cdots+\left(h_{\beta}-1\right) Q_{\beta}^{(1)}(\lambda, x) & +\cdots \\
& +h_{m} Q_{m}^{(1)}(\lambda, x)
\end{aligned}
$$

the truth of (13.13) will be inferred in consequence of (12.10a). If $h_{\beta}=0$ but some $h_{i}$, with $i<\beta$, is positive, write (13.13a); the validity of (13.13) is then deduced in view of (12.10) and (12.10a). The remaining case is the case when

$$
\begin{aligned}
Q(\lambda, x)= & -Q_{\beta}(\lambda, x)+h_{\beta+1} Q_{\beta+1}(\lambda, x)+\cdots+h_{m} Q_{m}(\lambda, x) \\
& \left(h_{\beta+1}, h_{\beta+2}, \cdots, h_{m} \geqq 0 ; h_{\beta+1}+\cdots+h_{m}=\nu \geqq 2 ; \beta<m\right) .
\end{aligned}
$$

$\dagger R\left(Q_{i}^{(1)}-Q_{\beta}^{(1)}\right) \leqq 0$ since $i<\beta$. 
In treating the case when (13.14) holds we make use of $(12.10 \mathrm{c})$. In view of (12.10) and (12.10c), whenever

$$
1 \leqq \beta<i \leqq j \leqq m,
$$

one has

$$
\begin{aligned}
R Q_{\beta}^{(1)}(\lambda, x) \geqq & {\left[R Q_{1}^{(1)}(\lambda, x) \geqq R Q_{m}^{(1)}(\lambda, x)+R Q_{m}^{(1)}(\lambda, x) \geqq\right] R Q_{i}^{(1)}(\lambda, x) } \\
& \left.+R Q_{i}^{(1)}(\lambda, x) \quad \text { (in } \Gamma\left(c, d ; R^{\prime}\right)\right) .
\end{aligned}
$$

Since in (13.14) $h_{\beta+1}+\cdots+h_{m} \geqq 2 \quad\left(h_{\beta+1}, \cdots, h_{m} \geqq 0\right)$,

$$
\begin{array}{r}
Q^{(1)}(\lambda, x)=\left[-Q_{\beta}^{(1)}(\lambda, x)+Q_{i}^{(1)}(\lambda, x)+Q_{i}^{(1)}(\lambda, x)\right]+k_{\beta+1} Q_{\beta}^{(1)}(\lambda, x) \\
+k_{\beta+2} Q_{\beta+2}^{(1)}(\lambda, x)+\cdots+k_{m} Q_{m}^{(1)}(\lambda, x) \quad\left(k_{\beta+1}, \cdots, k_{m} \geqq 0\right)
\end{array}
$$

for some pair of integers $i, j$ satisfying (13.15). By (13.15a) the real part of the expression within the brackets displayed above is equal to or is less than zero for $\lambda, x$ in $\Gamma\left(c, d ; R^{\prime}\right)$. Hence, in view of (13.16) and (12.10a), (13.13) will necessarily hold. Thus (13.13), as well as the lemma, has been established.

Henceforth the developments will be given for a region $\Gamma\left(c^{\prime}, d^{\prime} ; R^{\prime}\right)$, where $R^{\prime}$ is the same as in $\Gamma\left(c, d ; R^{\prime}\right)$, and where $\left(c^{\prime}, d^{\prime}\right)$ is an interval contained in $(c, d)$ such that in each of the expressions

$$
\begin{gathered}
Q^{(1)}(\lambda, x)=h_{1} Q_{1}^{(1)}(\lambda, x)+\cdots+h_{m} Q_{m}^{(1)}(\lambda, x)-Q_{\beta}^{(1)}(\lambda, x) \\
\left(h_{1} \geqq 0, \cdots, h_{m} \geqq 0 ; h_{1}+\cdots+h_{m}=\nu ; \text { the } h_{i} \text { integers } ; \beta=1, \cdots, n\right),
\end{gathered}
$$

formed for $\nu=2,3, \cdots, N$, the coefficient of the highest power of $\lambda$ is either $=0$ or $\neq 0$ throughout $\left(c^{\prime}, d^{\prime}\right)$. $\dagger$ The choice of $\left(c^{\prime}, d^{\prime}\right)$ may depend on $N$.

In view of Lemma 1 and of the relation (13.10) it is concluded that

$$
\begin{aligned}
\int{ }_{2}^{x} I_{\tau, \beta}(\lambda, u) d u=\exp \left[h_{1} G_{1}(\lambda,\right. & x)+\cdots \\
& \left.+h_{m} G_{m}(\lambda, x)-G_{\beta}(\lambda, x)\right] \lambda^{p+\omega}[\lambda, x]_{\bar{\alpha}-1}
\end{aligned}
$$

in $\Gamma\left(c^{\prime}, d^{\prime} ; R^{\prime}\right)$. Substitution of (13.17) in (13.4) (for $H=2$ ) will yield, in consequence of (13.1a),

$$
\begin{array}{r}
{ }_{2} \eta_{h_{1}}, \ldots, h_{m} ; j \\
(\lambda, x)=\exp [ \\
{\left[h_{1} G_{1}(\lambda, x)+\cdots+h_{m} G_{m}(\lambda, x)\right] \lambda^{p+\omega}[\lambda, x]_{\bar{\alpha}-1}} \\
\left(h_{1}+\cdots+h_{m}=2 ; \lambda, x \text { in } \Gamma\left(c^{\prime}, d^{\prime} ; R^{\prime}\right)\right) .
\end{array}
$$

By virtue of (13.6) and (13.18)

$\dagger$ When the coefficient of the highest power of $\lambda$ is $=0$ throughout $\left(c^{\prime}, d^{\prime}\right), Q^{(1)}(\lambda, x) \equiv 0$ (for $x$ in $c^{\prime}, d^{\prime}$ ). 


$$
\begin{aligned}
&{ }_{r} \eta_{k_{1}}^{r}, \cdots, k_{m}^{r}: \alpha \\
&(\lambda, x)=\exp \left[k_{1}^{r} G_{1}(\lambda, x)\right.+\cdots \\
&\left.+k_{m}^{r} G_{m}(\lambda, x)\right] \lambda^{\left(\nu_{r}-1\right)(p+\omega)}[\lambda, x]_{\bar{\alpha}-1} \\
&\left(k_{1}^{r}, \cdots, k_{m}^{r} \geqq 0 ; k_{1}^{r}+\cdots+\right.\left.k_{m}^{r}=\nu_{r} ; \alpha=1, \cdots, n ; \nu_{r}=1,2\right)
\end{aligned}
$$

for $\lambda, x$ in $\Gamma\left(c^{\prime}, d^{\prime} ; R^{\prime}\right)$. Substitute (13.19) in (12.15) (for $\theta=3$ ). Taking account of (12.17) we have

$$
\prod_{\tau=1}^{i_{\alpha}} \cdots=\exp \left[\delta_{1} G_{1}(\lambda, x)+\cdots+\delta_{m} G_{m}(\lambda, x)\right] \lambda^{\nu\left(\gamma_{\alpha^{\prime}} i_{\alpha}\right)(p+\omega)}[\lambda, x]_{\bar{\alpha}-1}
$$

where

$$
\nu\left(\gamma_{\alpha}, i_{\alpha}\right)=\sum_{r=1}^{i_{\alpha}}\left(\nu_{r}-1\right)=\gamma_{\alpha}-i_{\alpha}
$$

We next have

$F_{\alpha}^{\prime}=\sum_{\nu_{1}+\cdots+v_{i_{\alpha}=\gamma}} \sum^{(2)} \prod_{r=1}^{i_{\alpha}} \cdots=$ generic form of $(13.20) \quad\left(\right.$ in $\left.\Gamma\left(c^{\prime}, d^{\prime} ; R^{\prime}\right)\right)$.

If we write $\delta_{i}={ }_{a} \delta_{i}$, in consequence of the equalities of $(12.16 \mathrm{a})$, it is concluded that

(13.21) $\prod_{\alpha=1}^{n} F_{\alpha}^{\prime}=\exp \left[h_{1} G_{1}(\lambda, x)+\cdots+h_{m} G_{m}(\lambda, x)\right] \lambda^{\nu^{\prime}(H, s)(p+\omega)}[\lambda, x]_{\bar{\alpha}-1}$,

where

$$
\nu^{\prime}(3, s)=\sum_{\alpha=1}^{n}\left(\gamma_{\alpha}-i_{\alpha}\right)=3-s,
$$

by (12.16). The product of the functions of (13.21) by ${ }_{j} a_{i_{1}}, \ldots, i_{n}(\lambda, x)$ is a function possessing the generic form of the second member of (13.21). Hence

$$
F_{s}^{\prime \prime}=\sum_{i_{1}+\cdots+i_{n}=s} \sum_{\gamma_{1}+\cdots+\gamma_{n}=3} \sum_{\delta^{\delta_{1}} \cdots}^{(3)} \cdots=\text { generic form of (13.21). }
$$

Since, $\dagger$ for $s=2,3$, we have

$$
\lambda^{(3-s)(p+\omega)}[\lambda, x]_{\bar{\alpha}-1}=\lambda^{p+\omega}[\lambda, x]_{\bar{\alpha}-1},
$$

from (12.15) (for $B=3$ ) and (12.16) it follows that

$$
{ }_{3} \Gamma_{j}^{h_{1}, \cdots, h_{m}}=\sum_{s=2}^{3} F_{s}^{\prime \prime}=\exp \left[h_{1} G_{1}(\lambda, x)+\cdots+h_{m} G_{m}(\lambda, x)\right] \lambda^{p+\omega}[\lambda, x]_{\bar{\alpha}-1}
$$

$\dagger \lambda^{-(p+\omega)}[\lambda, x]_{\bar{\alpha}_{-1}}=[\lambda, x]_{\bar{\alpha}_{-1}}$.

$$
\left(h_{1}, \cdots, h_{m} \geqq 0 ; h_{1}+\cdots+h_{m}=3 ; \text { in } \Gamma\left(c^{\prime}, d^{\prime} ; R^{\prime}\right)\right) .
$$


Assume that $\nmid$ within $\Gamma\left(c^{\prime}, d^{\prime} ; R^{\prime}\right)$, for some ${ }_{B}(3 \leqq B \leqq N)$, we have

$$
\begin{gathered}
{ }_{q+1} \Gamma_{j}^{h_{1}, \cdots, h_{m}}=\exp \left[h_{1} G_{1}(\lambda, x)+\cdots+h_{m} G_{m}(\lambda, x)\right] \lambda^{(q-1)(p+\omega)}[\lambda, x]_{\bar{\alpha}-q+1} \\
\quad\left(h_{1}, \cdots, h_{m} \geqq 0 ; h_{1}+\cdots+h_{m}=q+1 ; j=1, \cdots, n\right), \\
{ }_{q} \eta_{k_{1}, \cdots, k_{m} ; \alpha}(\lambda, x) \\
=\exp \left[k_{1} G_{1}(\lambda, x)+\cdots+k_{m} G_{m}(\lambda, x)\right] \lambda^{(q-1)(p+\omega)}[\lambda, x]_{\bar{\alpha}-q+1} \\
\left(k_{1}, \cdots, k_{m} \geqq 0 ; k_{1}+\cdots+k_{m}=q ; \alpha=1, \cdots, n\right)
\end{gathered}
$$

for $q=1,2, \cdots, B-1$. For ${ }_{B}=3$ this has been previously established in (13.6), (13.9), (13.18), and (13.22).

Substitution of (13.23) (for $q=B-1$ ) in (13.4a) will yield

$$
\begin{aligned}
{ }_{H} I_{\tau, \beta}(\lambda, u) & \\
(13.25)= & \exp \left[h_{1} G_{1}(\lambda, u)+\cdots+h_{m} G_{m}(\lambda, u)-G_{\beta}(\lambda, u)\right] \lambda^{(H-1)(p+\omega)}[\lambda, u]_{\bar{\alpha}-H+2} \\
& \left(h_{1}, \cdots, h_{m} \geqq 0 ; h_{1}+\cdots+h_{m}={ }_{H} ; \lambda, u \text { in } \Gamma\left(c^{\prime}, d^{\prime} ; R^{\prime}\right)\right) .
\end{aligned}
$$

Since $h_{1}+\cdots+h_{m}=B \leqq N$, in view of the italicized statement preceding (13.17) it is observed that Lemma 1 (together with the appended note) is applicable for the evaluation of

$$
\int_{H}^{x} I_{\tau, \beta}(\lambda, u) d u \text {. }
$$

Accordingly (13.25a) will have the form

$$
\exp \left[h_{1} G_{1}(\lambda, u)+\cdots+h_{m} G_{m}(\lambda, u)-G_{\beta}(\lambda, u)\right] \lambda^{(H-1)(p+\omega)}[\lambda, x]_{\bar{\alpha}-H+1} .
$$

Substitution of this in (13.4) will give

$$
\begin{array}{r}
{ }_{H} \eta_{h_{1}}, \cdots, h_{m} ; j \\
\quad \\
=\exp \left[h_{1} G_{1}(\lambda, x)+\cdots+h_{m} G_{m}(\lambda, x)\right] \lambda^{(H-1)(p+\omega)}[\lambda, x]_{\bar{\alpha}-H+1} \\
\left(\lambda, x \text { in } \Gamma\left(c^{\prime}, d^{\prime} ; R^{\prime}\right)\right) .
\end{array}
$$

Thus (13.24) holds for $q=1,2, \cdots, \boldsymbol{B}$.

In (12.15) replace $\theta$ by $\theta+1$ and substitute (13.24) for $q=1,2, \cdots, B$. Since (12.17) holds, and since $\nu_{1}+\cdots+\nu_{i_{\alpha}}=\gamma_{\alpha}$,

$$
\prod_{r=1}^{i_{\alpha}} \cdots=\exp \left[\delta_{1} G_{1}(\lambda, x)+\cdots+\delta_{m} G_{m}(\lambda, x)\right] \lambda\left(\gamma_{\alpha}-i_{\alpha}\right)(p+\omega)[\lambda, x]_{\bar{\alpha}-H+1} .
$$

We next obtain

$$
F_{\alpha}^{\prime}=\sum_{v_{1}+\cdots+v_{i_{\alpha}}=\gamma} \sum^{(2)} \prod_{r=1}^{i_{\alpha}} \cdots=\text { generic form of }(13.27) \quad\left(\delta_{i}={ }_{\alpha} \delta_{i}\right),
$$

† We take $\alpha$ (hence $\bar{\alpha}$ also) suitably great. 
and, by (12.16a) and (12.16) (with $\boldsymbol{\theta}$ replaced by $\boldsymbol{\theta}+1$ ),

$$
\begin{gathered}
\prod_{\alpha=1}^{n} F_{\alpha}^{\prime}=\exp \left[h_{1} G_{1}(\lambda, x)+\cdots+h_{m} G_{m}(\lambda, x)\right] \lambda^{(H+1-s)(p+\omega)}[\lambda, x]_{\bar{\alpha}-H+1} \\
=\exp \left[h_{1} G_{1}(\lambda, x)+\cdots+h_{m} G_{m}(\lambda, x)\right] \lambda^{(H-1)(p+\omega)}[\lambda, x]_{\bar{\alpha}-H+1} \\
\left(s=2, \cdots, B+1 ; \text { in } \Gamma\left(c^{\prime}, d^{\prime} ; R^{\prime}\right)\right) .
\end{gathered}
$$

Finally, by (12.16) (with $\theta$ replaced by $z+1$ ),

$$
\begin{aligned}
{ }_{H+1} \Gamma_{j}^{h_{1}, \cdots, h_{m}} & =\sum_{j}^{(4)} a_{i_{1}, \cdots, i_{n}}(\lambda, x) \prod_{\alpha=1}^{n} F_{\alpha}{ }^{\prime} \\
& =\text { generic form of third member of (13.27a). }
\end{aligned}
$$

Thus relations (13.23), (13.24) are valid in $\Gamma\left(c^{\prime}, d^{\prime} ; R^{\prime}\right)$ for $q=1,2, \cdots, N$.

LEMмa 2. Let $N$ be an integer, however large. Let $\Gamma\left(c, d ; R^{\prime}\right)$ be an admissible region (cf. Definition $3, \S 12$ ) and $\Gamma\left(\alpha^{\prime}, \beta^{\prime} ; R\right.$ ) be a proper region (cf. Definition $2, \S 12)$ containing $\Gamma\left(c, d ; R^{\prime}\right)$. With suitable notation we thus have (12.10), (12.10a), (12.10b), and (12.10c).

Let $\left(c^{\prime}, d^{\prime}\right)$ be an interval contained in $(c, d)$ and satisfying the condition of the italicized statement preceding (13.17).

- The system (11.1) possesses a formal solution represented by the series $s_{j}(\lambda, x)(j=1, \cdots, n)$ given by (12.13) and (12.13a). The coefficients

$$
{ }_{\nu} \eta_{k_{1}, \cdots, k_{m} ; j}(\lambda, x) \quad\left(k_{1}, \cdots, k_{m} \geqq 0 ; k_{1}+\cdots+k_{m}=\nu\right),
$$

involved in (12.13a) can be determined by means of (13.4), (13.4a) and the formulas (12.15), (12.16), and (12.17). For $\nu=1,2, \cdots, N$ the functions (13.28) may be taken of the form

$$
\begin{aligned}
& { }_{\nu} \eta_{k_{1}, \cdots, k_{m} ; j}(\lambda, x) \\
& =\exp \left[k_{1} G_{1}(\lambda, x)+\cdots+k_{m} G_{m}(\lambda, x)\right] \lambda^{(\nu-1)(p+\omega)}[\lambda, x]_{\bar{\alpha}-\nu+1} \\
& \quad\left(k_{1}, \cdots, k_{m} \geqq 0 ; k_{1}+\cdots+k_{m}=\nu ; j=1, \cdots, n\right),
\end{aligned}
$$

formulas (13.28a) being valid in $\Gamma\left(c^{\prime}, d^{\prime} ; R^{\prime}\right)$. The symbol $[\lambda, x]_{\bar{\alpha}-\nu+1}$ is defined (in $\Gamma\left(c^{\prime}, d^{\prime} ; R^{\prime}\right)$ ) according to Definition 4 . We may take $\bar{\alpha}$ as great as desired; however, the functions (13.28) will in general depend on $\bar{\alpha}$.

14. Corresponding transformations. The system (11.1) will be transformed with the aid of

$$
y_{j}(x)=Y_{j}(\lambda, x)+\rho_{j}(\lambda, x)
$$

$$
(j=1, \cdots, n)
$$

where

$$
Y_{j}(\lambda, x)={ }_{1} y_{j}(\lambda, x)+{ }_{2} y_{j}(\lambda, x)+\cdots+{ }_{N-1} y_{j}(\lambda, x)
$$


Here the ${ }_{\nu} y_{j}(\lambda, x)(\nu=1, \cdots, N-1)$ are the functions involved in the formal series solutions $s_{j}(\lambda, x)$, referred to in Lemma $2, \S 13$. The arbitrary constants $c_{1}, \cdots, c_{m}$, involved in the ${ }_{\nu} y_{j}(\lambda, x)$ may be taken as functions of $\lambda . W e$ shall take the $c_{i}=c_{i}(\lambda)$ analytic in $R^{\prime}(\lambda \neq \infty)$ and such that

$$
\left|c_{i}\right| \leqq \gamma(\lambda) ; \gamma(\lambda) \sim 0 ; \quad \gamma(\lambda) \leqq \gamma_{1} \quad\left(i=1, \cdots, m ; \lambda \text { in } R^{\prime}\right) .
$$

In view of (12.10b) it follows from (13.28a) that

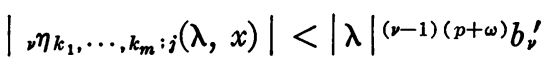

$$
\left(k_{1}, \cdots, k_{m} \geqq 0 ; k_{1}+\cdots+k_{m}=\nu ; j=1, \cdots, n ; \nu=1,2, \cdots, N-1\right)
$$

for $\lambda, x$ in $\Gamma\left(c^{\prime}, d^{\prime} ; R^{\prime}\right)$. Here $b_{\nu}^{\prime}$ is independent of $\lambda$ and $x$. By (14.2), (14.3), and (12.13a)

$$
{ }_{\nu} y_{j}(\lambda, x) \sim 0 ; \quad\left|{ }_{\nu} y_{j}(\lambda, x)\right|<\gamma^{\nu}(\lambda)|\lambda|^{(\nu-1)(p+\omega)} b_{\nu}{ }^{\prime \prime} \quad\left(\text { in } \Gamma\left(c^{\prime}, d^{\prime} ; R^{\prime}\right)\right) .
$$

Hence, since $\gamma(\lambda) \sim 0$,

$$
Y_{j}(\lambda, x) \sim 0 ; \quad\left|Y_{j}(\lambda, x)\right|<\gamma(\lambda) b^{\prime}
$$$$
\text { (in } \Gamma\left(c^{\prime}, d^{\prime} ; R^{\prime}\right) \text { ). }
$$

Here $b^{\prime}$ is independent of $x, \lambda$. Either by taking $\gamma_{1}(>0)$ sufficiently small or $c^{\prime}$ sufficiently great, we secure

$$
\left|Y_{j}(\lambda, x)\right| \leqq \rho^{\prime} \quad\left(j=1, \cdots, n ; \text { in } \Gamma\left(c^{\prime}, d^{\prime} ; R^{\prime}\right) ;|\lambda| \geqq r^{\prime}\right),
$$

where $\rho^{\prime}$ is independent of $\lambda, x$ and

$$
0<\rho^{\prime}<\rho,
$$

where $\rho$ is defined as in (11.4).

In consequence of (11.1a) and (11.1b) substitution of (14.1) in (11.1) will yield

$$
\begin{aligned}
\lambda^{-p} \rho_{j}^{(1)}(\lambda, x)- & l_{j}\left(\lambda, x, \rho_{1}, \cdots, \rho_{n}\right) \\
= & -\lambda^{-p} Y_{j}^{(1)}(\lambda, x)+l_{j}\left(\lambda, x, Y_{1}, \cdots, Y_{n}\right) \\
& +q_{j}\left(\lambda, x, Y_{1}+\rho_{1}, \cdots, Y_{n}+\rho_{n}\right) .
\end{aligned}
$$

Here

$$
\begin{aligned}
& q_{j}\left(\lambda, x, Y_{1}+\rho_{1}, \cdots\right)=q_{j}\left(\lambda, x, Y_{1}, \cdots, Y_{n}\right) \\
& +\sum_{\nu_{1}+\cdots+\nu_{n} \geqq 1} \alpha_{\nu_{1}}, \cdots, \nu_{n} ; j(\lambda, x) \rho_{1}^{\nu_{1}} \cdots \rho_{n}^{\nu_{n}}, \\
& \left.\nu_{1} ! \cdots \nu_{n} ! \alpha_{\nu_{1}} \cdots, p_{n} ; j(\lambda, x)=\frac{\partial^{\nu_{1}+\cdots+\nu_{n}}}{\partial^{\nu_{1}} y_{1} \cdots \partial^{\nu_{n}} y_{n}} q_{j}\left(\lambda, x, y_{1}, \cdots, y_{n}\right)\right] \\
& \left(y_{i}=Y_{i}(\lambda, x) ; i=1, \cdots, n\right),
\end{aligned}
$$


the series above being absolutely and uniformly convergent in $\Gamma\left(c^{\prime}, d^{\prime} ; R^{\prime}\right)$ $\left(|\lambda| \geqq r^{\prime}\right)$ provided

$$
\left|\rho_{1}\right|, \cdots,\left|\rho_{n}\right| \leqq \rho^{\prime \prime} \quad\left(0<\rho^{\prime \prime}<\rho-\rho^{\prime}\right)
$$

(cf. $(14.4 \mathrm{~b})) . \dagger$

By (14.5b) and (11.1c)

$$
\begin{array}{r}
\alpha_{\nu_{1}}, \cdots, \nu_{n} \\
(\lambda, x)=\sum_{\lambda_{1}, \cdots, \lambda_{n}} a_{\nu_{1}+\lambda_{1}, \cdots, \nu_{n}+\lambda_{n}}(\lambda, x) C_{\lambda_{1}}^{{ }^{{ }_{1}+\lambda_{1}}} \cdots C_{\lambda_{n}}^{\nu_{n}+\lambda_{n}} Y_{1}^{\lambda_{1}} \cdots Y_{n}^{\lambda_{m}} \\
\left(\lambda_{1}, \cdots, \lambda_{n} \geqq 0 ; \lambda_{1}+\cdots+\lambda_{n} \geqq 2-\left(\nu_{1}+\cdots+\nu_{n}\right)\right) .
\end{array}
$$

Thus, in particular,

$$
\alpha_{\nu_{1}}, \cdots, \nu_{n} ; j(\lambda, x)=\sum_{\lambda_{1}+\cdots+\lambda_{n} \geqq 1} \cdots \quad\left(\nu_{1}+\cdots+\nu_{n}=1\right),
$$

so that, by (14.7) and (14.4),

$$
\alpha_{\nu_{1}, \cdots, \nu_{n} ; j}(\lambda, x) \sim 0 \quad\left(\text { in } \Gamma\left(c^{\prime}, d^{\prime} ; R^{\prime}\right) ; \nu_{1}+\cdots+\nu_{n}=1\right) .
$$

On the other hand,

$$
\alpha_{\nu_{1}, \cdots, \nu_{n} ; j}(\lambda, x)={ }_{j} a_{\nu_{1}, \cdots, \nu_{n}}(\lambda, x)+\sum_{\lambda_{1}+\cdots+\lambda_{n} \geqq 1} \cdots \quad\left(\nu_{1}+\cdots+\nu_{n} \geqq 2\right) ;
$$

thus, in view of (14.7),

$$
\alpha_{\nu_{1}, \cdots, \nu_{n} ; j}(\lambda, x)={ }_{j} a_{\nu_{1}, \cdots, \nu_{n}}(\lambda, x)+{ }_{j} \beta_{\nu_{1}, \cdots, \nu_{n}}(\lambda, x),
$$

where

$$
{ }_{j} \beta_{\nu_{1}, \cdots, \nu_{n}}(\lambda, x) \sim 0 \quad\left(\nu_{1}+\cdots+\nu_{n} \geqq 2 ; \text { in } \Gamma\left(c^{\prime}, d^{\prime} ; R^{\prime}\right)\right) .
$$

By (14.5a), (14.7a), (14.7b), (14.7c), and (14.5)

\section{Here}

$$
\begin{array}{r}
\lambda^{-p} \rho_{j}^{(1)}(\lambda, x)-l_{j}^{*}\left(\lambda, x, \rho_{1}, \cdots, \rho_{n}\right)=q_{j}^{*}\left(\lambda, x, \rho_{1}, \cdots, \rho_{n}\right)-F_{j}(\lambda, x) \\
(j=1, \cdots, n) .
\end{array}
$$

(14.8a) $F_{j}(\lambda, x)=\lambda^{-p} Y_{j}^{(1)}(\lambda, x)-l_{j}\left(\lambda, x, Y_{1}, \cdots, Y_{n}\right)-q_{j}\left(\lambda, x, Y_{1}, \cdots, Y_{n}\right)$ and

$$
\left.l_{j}^{*} \sim l_{j} ; q_{j}^{*} \sim q_{j} \quad \text { (in } \Gamma\left(c^{\prime}, d^{\prime} ; R^{\prime}\right)\right) .
$$

The relations (14.9) are to be understood as follows. The $l_{j}^{*}-l_{j}$ are linear in $\rho_{1}, \cdots, \rho_{n}$ and the $q_{j}^{*}-q_{j}$ are series in non-negative integral powers of $\rho_{1}, \cdots, \rho_{n}$; the coefficients of the various powers of $\rho_{1}, \cdots, \rho_{n}$ are functions of $x$ and $\lambda$ which are all $\sim 0$ in $\Gamma\left(c^{\prime}, d^{\prime} ; R^{\prime}\right)$.

$\dagger \rho^{\prime \prime}$ is independent of $x$ and $\lambda$. 
It remains to study the function (14.8a). By (12.18) and (12.18a) in consequence of $(14.8 \mathrm{a})$ we have

where

$$
\begin{aligned}
F_{j}(\lambda, x) & =\lambda^{-p}\left[Y_{j}^{(1)}(\lambda, x)-\lambda^{p} a_{j}\left(\lambda, x, Y_{1}, \cdots, Y_{n}\right)\right] \\
& =\sum_{H=2}^{\infty} \sum_{h_{1}+\cdots+h_{m}=H} c_{1}^{h_{1}} \cdots c_{m}^{h_{m}} \lambda^{-p}{ }_{H} \psi_{h_{1}}^{*}, \cdots, h_{m} ; j
\end{aligned}
$$

$$
{ }_{H} \psi_{h_{1}, \cdots, h_{m} ; j}^{*}={ }_{H} \psi_{h_{1}, \cdots, h_{m} ; j},
$$

provided that in the second member of (14.11) we replace the

$$
{ } \eta_{k_{1}}, \cdots, k_{m} ; \alpha(\lambda, x)
$$

by zeros. $\uparrow$ Thus by $(12.18 \mathrm{a})$

$$
\begin{array}{rlrl}
{ }_{H} \psi_{h_{1}}^{*}, \cdots, h_{m} ; j & ={ }_{H} \psi_{h_{1}, \cdots, h_{m} ; j} & (B \leqq N-1), \\
{ }_{N} \psi_{h_{1}, \cdots, h_{m} ; j}^{*}=-\lambda^{p}{ }_{N} \Gamma_{j}^{h_{1}, \cdots, h_{m}} & \\
{ }_{H} \psi_{h_{1}}^{*}, \cdots, h_{m} ; j & =-\lambda{ }^{p}{ }_{H} \Gamma_{j}^{h_{1}, \cdots, h_{m}}, &
\end{array}
$$

In $(14.12 \mathrm{c})$

$$
{ }_{H} \Gamma_{j}^{h_{1}, \cdots, h_{m}, *}={ }_{H} \Gamma_{j}^{h_{1}, \cdots, h_{m}} \quad(B>N),
$$

provided that in the second members the functions (14.12) are replaced by zeros.

It is to be recalled that the

$$
\begin{aligned}
& { }_{\nu} \eta_{k_{1}}, \cdots, k_{m}: \alpha(\lambda, x) \\
& (\nu \leqq N-1)
\end{aligned}
$$

have been previously determined so that

$$
{ }_{H} \psi_{h_{1}, \cdots, h_{m} ; j}=0 \quad(B \leqq N-1) .
$$

If we take account of the statement preceding Lemma $2, \S 13$, the form of the function of (14.12b) is inferred with the aid of (13.23) (for $q=N-1$ ). Thus,

$$
\begin{aligned}
& { }_{N} \psi_{h_{1}, \cdots, h_{m} ; j}^{*} \\
= & \exp \left[h_{1} G_{1}(\lambda, x)+\cdots+h_{m} G_{m}(\lambda, x)\right] \lambda^{(N-2)(p+\omega)+p}[\lambda, x]_{\bar{\alpha}-N+2} \\
& \left(h_{1}+\cdots+h_{m}=N ; \text { in } \Gamma\left(c^{\prime}, d^{\prime} ; R^{\prime}\right)\right) .
\end{aligned}
$$

The form of the functions (14.13) can be established as follows.

$\dagger$ When the functions (14.12) have been replaced by zeros, the formal series $s_{j}(\lambda, x)={ }_{1} y_{j}(\lambda, x)$ $+{ }_{2} y_{j}(\lambda, x)+\cdots$ reduce to the functions $Y_{j}(\lambda, x)$, respectively. On the other hand, (12.18) and (12.18a) have been obtained as a result of substituting in $y_{j}^{(1)}(\lambda, x)-\lambda^{p} a_{j}\left(\lambda, x, y_{1}, \cdots, y_{n}\right)$ the series $s_{i}(\lambda, x)$. 
Since, for $\nu \leqq N-1, \bar{\alpha}-\nu+1 \geqq \bar{\alpha}-N+2$, in consequence of (13.24) (for $q=1, \cdots, N-1)$ we have

$$
\begin{array}{r}
{ }_{\nu} \eta_{k_{1}}, \cdots, k_{m} ; \alpha \\
(\lambda, x)=\exp \left[k_{1} G_{1}+\cdots+k_{m} G_{m}\right] \lambda^{(\nu-1)(p+\omega)}[\lambda, x]_{\bar{\alpha}-N+2} \\
\left(k_{1}+\cdots+k_{m}=\nu ; \nu=1, \cdots, N-1 ; \text { in } \Gamma\left(c^{\prime}, d^{\prime} ; R^{\prime}\right)\right) .
\end{array}
$$

With the functions (14.12) replaced by zeros, we may write

$$
\begin{array}{r}
{ }_{\nu} \eta_{k_{1}}, \cdots, k_{m} ; \alpha \\
\left(k_{1}+\cdots+k_{m}=\nu ; \nu=N, N+1, \cdots ; \text { in } \Gamma\left(c^{\prime}, d^{\prime} ; R^{\prime}\right)\right) .
\end{array}
$$

If (14.15) and (14.15a) are substituted in (12.15), in view of the definition of the functions (14.13) it is inferred that

$$
\begin{array}{r}
{ }_{H} \Gamma_{j}^{h_{1}} ; \cdots, h_{m},^{*}=\exp \left[h_{1} G_{1}(\lambda, x)+\cdots+h_{m} G_{m}(\lambda, x)\right] \lambda^{(H-2)(p+\omega)}[\lambda, x]_{\bar{\alpha}-N+2} \\
\left(h_{1}+\cdots+h_{m}={ }_{B} ; H=N+1, N+2, \cdots ; \text { in } \Gamma\left(c^{\prime}, d^{\prime} ; R^{\prime}\right)\right) .
\end{array}
$$

By (14.14)

$$
F_{j}(\lambda, x)=\sum_{H \geqq N} \sum_{h_{1}+\cdots+h_{m}=H} \lambda^{-p c_{1}^{h_{1}}} \cdots c_{m H}^{h_{m}} \psi_{h_{1}, \cdots, h_{m} ; j}^{*}
$$

Thus, in consequence of (14.2), (14.14a), (14.12c), and (14.16)

$$
\begin{array}{r}
\left|F_{j}(\lambda, x)\right|<k_{0}(\gamma(\lambda))^{N}|\lambda|^{(N-2)(p+\omega)} \sum_{h_{1}+\cdots+h_{m}=N} g_{h_{1}, \cdots, h_{m}}(\lambda, x) \\
\left(j=1, \cdots, n ; \text { in } \Gamma\left(c^{\prime}, d^{\prime} ; R^{\prime}\right)\right),
\end{array}
$$

where

$$
g_{h_{1}, \cdots, h_{m}}(\lambda, x)=\left|\exp \left[h_{1} G_{1}(\lambda, x)+\cdots+h_{m} G_{m}(\lambda, x)\right]\right| .
$$

LEMma 3. The transformation (14.1), (14.1a) (cf. the italics subsequent to (14.1a)), applied to the system (11.1), will yield the system

$$
\begin{array}{r}
\lambda^{-p} \rho_{j}^{(1)}(\lambda, x)-l_{j}^{*}\left(\lambda, x, \rho_{1}, \cdots, \rho_{n}\right)=q_{i}^{*}\left(\lambda, x, \rho_{1}, \cdots, \rho_{n}\right)-F_{j}(\lambda, x) \\
(j=1, \cdots, n) .
\end{array}
$$

The $l_{j}^{*}$ and $q_{j}^{*}$, here involved, satisfy (14.9) in the sense of the italicized statement subsequent to (14.9). The series

(14.19a) $\quad q_{j}^{*}\left(\lambda, x, \rho_{1}, \cdots, \rho_{n}\right)=\sum_{\nu_{1}+\cdots+\nu_{n} \geqq 2} \alpha_{\nu_{1}}, \cdots, \nu_{n} ; j(\lambda, x) \rho_{1}^{\nu_{1}} \cdots \rho_{n}^{\nu_{n}}$

will converge absolutely and uniformly in $\Gamma\left(c^{\prime}, d^{\prime} ; R^{\prime}\right)\left(|\lambda| \geqq r^{\prime}\right)$, provided (14.6) holds. Moreover, the $F_{j}(\lambda, x)$ satisfy (14.17), (14.18). The above is asserted under the supposition that the arbitrary constants (functions of $\lambda$ ) $c_{i}$ satisfy (14.2); furthermore, $\gamma_{1}$ and $r^{\prime}$ are to be suitably chosen so that (14.4a), (14.4b) hold. 
15. The Third Existence Theorem. We now have the following statement which is analgous to a statement in $\$ 7$.

Let $\lambda, x$ be in $\Gamma\left(c^{\prime}, d^{\prime} ; R^{\prime}\right)\left(|\lambda| \geqq r^{\prime}\right)$. Suppose

$$
\left|\rho_{i}\right| \leqq \rho^{*}, \quad\left|w_{i}\right| \leqq w^{*} \quad(i=1, \cdots, n)
$$

with

$$
\rho^{*}+w^{*} \leqq \rho^{\prime \prime} .
$$

For the function (14.19a) we then shall have

$$
\begin{aligned}
& \left|q_{j}^{*}\left(\lambda, x, \rho_{1}+w_{1}, \cdots, \rho_{n}+w_{n}\right)-q_{j}^{*}\left(\lambda, x, \rho_{1}, \cdots, \rho_{n}\right)\right| \\
& \quad<\left(q^{\prime} \rho^{*}+q w^{*}\right) w^{*} \quad\left(\lambda, x \text { in } \Gamma\left(c^{\prime}, d^{\prime} ; R^{\prime}\right) ; q^{\prime}, q \text { independent of } \lambda, x\right) .
\end{aligned}
$$

We now write

$$
\begin{aligned}
& \rho_{j: k}-\rho_{j: k-1}=w_{j: k} \\
& \rho_{j: k}=w_{j: 0}+w_{j: 1}+\cdots+w_{j: k}
\end{aligned} \quad\left(k=0,1, \cdots ; \rho_{j:-1}=0\right),
$$

and consider the succession of differential systems

$$
\begin{aligned}
& \lambda^{-p} w_{j: k}^{(1)}-l_{j}^{*}\left(\lambda, x, w_{1: k}, \cdots, w_{n: k}\right)=g_{j: k} \\
& \equiv q_{j}^{*}\left(\lambda, x, \rho_{1: k-2}+w_{1: k-1}, \cdots, \rho_{n: k-2}+w_{n: k-1}\right) \\
& -q_{j}^{*}\left(\lambda, x, \rho_{1: k-2}, \cdots, \rho_{n: k-2}\right) \\
& \quad\left(j=1, \cdots, n ; k=0,1, \cdots ; g_{j: 0}=-{ }_{j} F(\lambda, x)\right) .
\end{aligned}
$$

A solution will be obtained in the form of convergent series

$$
\rho_{j}(\lambda, x)=w_{j: 0}(\lambda, x)+w_{j: 1}(\lambda, x)+\cdots \quad(j=1, \cdots, n) .
$$

The left member in (15.4), multiplied by $\lambda^{p}$, is "asymptotically" identical with the left member in (12.19) (cf. Lemma 3, \$14). Hence in consequence of the statement in connection with (12.19) $-(12.20 \mathrm{~b})$ one may write (15.4) in the form

$$
w_{j: k}(\lambda, x)=\sum_{\beta=1}^{n}{ }_{k} c_{\beta}(\lambda, x)_{0} y_{\beta, j}^{*}(\lambda, x) \quad(j=1, \cdots, n),
$$

where

$$
{ }_{k} c_{\beta}(\lambda, x)=\sum_{\tau=1}^{n} \int^{x} \lambda^{p} g_{\tau: k} \bar{y}_{\tau, \beta}^{*}(\lambda, u) d u
$$

Here the ${ }_{0} y_{i, j}^{*}(\lambda, x)$ can be taken asymptotically the same (in $\Gamma\left(c^{\prime}, d^{\prime} ; R^{\prime}\right)$ ) as the ${ }_{0} y_{i, j}(\lambda, x)(i, j=1, \cdots, n)$, involved in (12.20); moreover,

$$
\left({ }_{0} \bar{y}_{i, j}^{*}(\lambda, u)\right)=\left({ }_{0} y_{i, j}^{*}(\lambda, u)\right)^{-1} .
$$


If account is taken of (13.1), (13.2), and (13.3), it is concluded that

$$
\begin{array}{rlrl}
{ }_{0} y_{\beta, j}^{*}(\lambda, x) & =e^{G_{\beta}(\lambda, x)} \gamma_{\beta, j}^{*}(\lambda, x), & \gamma_{\beta, j}^{*}(\lambda, x) & =[\lambda, x]_{\sigma}, \\
{ }_{0} \bar{y}_{\tau, \beta}^{*}(\lambda, u) & =e^{-G_{\beta}(\lambda, u)} \lambda^{\omega} \bar{\gamma}_{\tau, \beta}^{*}(\lambda, u), & \bar{\gamma}_{\tau, \beta}^{*}(\lambda, u)=[\lambda, u]_{\bar{\sigma}}, \\
\left|\gamma_{\beta, j}^{*}(\lambda, x)\right|, & \left|\bar{\gamma}_{\tau, \beta}^{*}(\lambda, x)\right|<\gamma_{*} & & \text { (in } \left.\Gamma\left(c^{\prime}, d^{\prime} ; R^{\prime}\right)\right) .
\end{array}
$$

Moreover, $\sigma$ can be taken as great as desired, and $\bar{\sigma} \rightarrow \infty$ with $\sigma$. However, the $y_{i, j}^{*}(\lambda, x)$ will, in general, depend on $\sigma$.

In view of the above (15.6) may be written in the form

$$
\begin{aligned}
w_{j: k}(\lambda, x) & =\sum_{\beta=1}^{n}{ }_{k} c_{\beta}(\lambda, x) e^{G_{\beta}(\lambda, x)} \gamma_{\beta, j}^{*}(\lambda, x), \\
{ }_{k} c_{\beta}(\lambda, x) & =\sum_{\tau=1}^{n} \int^{x} \lambda^{p+\omega} g_{\tau: k} e^{-G_{\beta}(\lambda, u)} \bar{\gamma}_{\tau, \beta}^{*}(\lambda, u) d u .
\end{aligned}
$$

Finally, with the aid of $(15.7 \mathrm{~b})$ we obtain

$$
\left|w_{j: k}(\lambda, x)\right|<n \gamma^{2} \sum_{\beta=1}^{n}\left|e^{G_{\beta}(\lambda, x)}\right| \int^{x} g_{k}|\lambda| p+\omega\left|e^{-G_{\beta}(\lambda, u)}\right||d u|
$$

(in $\left.\Gamma\left(c^{\prime}, d^{\prime} ; R\right)\right)$, provided $\left|g_{j: k}\right| \leqq g_{k}(j=1, \cdots, n)$ and the integrals here involved can be evaluated.

Write

$$
\begin{aligned}
& q_{\nu}(\lambda, x)=\sum_{h_{1}+\cdots+h_{m}=\nu}\left|\exp \left[h_{1} G_{1}(\lambda, x)+\cdots+h_{m} G_{m}(\lambda, x)\right]\right|, \\
& \mu(\lambda)=\gamma(\lambda)|\lambda|^{p+\omega} .
\end{aligned}
$$

Then, since $g_{j: 0}=-{ }_{j} F(\lambda, u)$, in view of (14.17) we may take

$$
g_{0}=k_{0} \mu^{N}(\lambda)|\lambda|^{-2(p+\omega)} q_{N}^{(\lambda, u)} .
$$

Whence, by (15.9),

$$
\begin{aligned}
\left|w_{j: 0}(\lambda, x)\right|<k_{0} n \gamma^{2} \mu^{N}(\lambda)|\lambda|-(p+\omega) & \sum_{\beta=1}^{n}\left|e^{G \beta(\lambda, x)}\right| \\
\cdot & \sum_{h_{1}+\cdots+h_{m}=N} \int^{x}\left|e^{G(\lambda, u)}\right||d u|,
\end{aligned}
$$

where

$$
G(\lambda, u)=h_{1} G_{1}(\lambda, u)+\cdots+h_{m} G_{m}(\lambda, u)-G_{\beta}(\lambda, u) .
$$

Now

(15.13a) $\quad R G^{(1)}(\lambda, u)=h_{1} R Q_{1}^{(1)}(\lambda, u)+\cdots+h_{m} R Q_{m}^{(1)}(\lambda, u)-R Q_{\beta}^{(1)}(\lambda, u)$. 
Applications of (12.10), (12.10a), and (12.10c) leads (by the same reasoning as employed in proving (13.13)) to the conclusion that the function of (15.13a) is $\leqq 0$ for $\lambda, u$ in $\Gamma\left(c^{\prime}, d^{\prime} ; R^{\prime}\right)$. $\dagger$ Since

and since, for

$$
R[G(\lambda, x)-G(\lambda, u)]=\int_{u}^{x} R G^{(1)}(\lambda, v) d v
$$

$$
c^{\prime} \leqq x \leqq v \leqq u \leqq d^{\prime},
$$

$d v \leqq 0$, the integrand just displayed is $\geqq 0$ for $\lambda, x$ in $\Gamma\left(c^{\prime}, d^{\prime} ; R^{\prime}\right)$, provided (15.14) holds. Thus, under (15.14),

$$
R G(\lambda, x) \geqq R G(\lambda, u)
$$

(cf. (15.13)). Accordingly,

$$
\int_{d^{\prime}}^{x}\left|e^{G(\lambda, u)} d u\right| \leqq\left|e^{G(\lambda, x)}\right| \int_{d^{\prime}}^{x}|d u|=\left|e^{G(\lambda, x)}\right|\left(d^{\prime}-c^{\prime}\right)
$$

$$
\left(\lambda, x \text { in } \Gamma\left(c^{\prime}, d^{\prime} ; R^{\prime}\right)\right) \text {. }
$$

In the sequel integrations will be from $d^{\prime}$ to $x\left(x\right.$ in $\left.\left(c^{\prime}, d^{\prime}\right)\right)$.

By (15.15), (15.12), (15.13), and the definition of $q_{N}(\lambda, x)$

$$
\begin{aligned}
\left|w_{j: 0}(\lambda, x)\right| & <k_{0} k_{1} \mu^{N}(\lambda)|\lambda|^{-(p+\omega)} q_{N}(\lambda, x)=k_{1}|\lambda|{ }^{p+\omega} g_{0}(\lambda, x) \\
& =w_{0}^{*}(\lambda, x) \quad\left(j=1, \cdots, n ; \lambda, x \text { in } \Gamma\left(c^{\prime}, d^{\prime} ; R^{\prime}\right)\right)
\end{aligned}
$$

(cf. (15.11)), where

$$
k_{1}=\left(d^{\prime}-c^{\prime}\right) n^{2} \gamma^{2} .
$$

With $q^{\prime}$ and $q$ denoting the constants of (15.2) let $b$ be the upper bound of the numbers

$$
q, \quad q^{\prime}\left[1+\frac{\rho^{\prime \prime}}{1+\rho^{\prime \prime}}+\cdots+\left(\frac{\rho^{\prime \prime}}{1+\rho^{\prime \prime}}\right)^{\nu-1}\right]+q\left(\frac{\rho^{\prime \prime}}{1+\rho^{\prime \prime}}\right)^{\nu}
$$

$(\nu=1,2, \cdots) ; b$ will be finite. The inequalities

$$
\begin{array}{r}
w_{0}^{*}(\lambda, x) \leqq \frac{\rho^{\prime \prime}}{1+\rho^{\prime \prime}}, \quad \Gamma(\lambda, x)=k^{\prime} b|\lambda|^{p+\omega} w_{0}^{*}(\lambda, x) \leqq \frac{\rho^{\prime \prime}}{1+\rho^{\prime \prime}} \\
\left(\lambda, x \text { in } \Gamma\left(c^{\prime}, d^{\prime} ; R^{\prime}\right)\left(|\lambda| \geqq r^{\prime}\right)\right)
\end{array}
$$

(cf. (15.16), (15.16a)) will be obtained either by

(i) taking $r^{\prime}$ sufficiently great;

or by

† Presently, however, it is immaterial whether the coefficient of the highest power of $\lambda$, in (15.13a), vanishes for any $x$ in $\left(c^{\prime}, d^{\prime}\right)$. 
(ii) taking the interval $\left(c^{\prime}, d^{\prime}\right)$ sufficiently small.

If we make use of (15.4) for $k=1$ (with $\rho_{j:-1}=0$ ) and apply (15.1), (15.2), with

$$
\rho^{*}=\rho_{-1}^{*}(\lambda, x)=0, \quad w^{*}=w_{0}^{*}(\lambda, x),
$$

it follows that

$$
\left|g_{j: 1}(\lambda, u)\right|<b\left(w_{0}^{*}(\lambda, u)\right)^{2}=g_{1} \quad\left(\lambda, u \text { in } \Gamma\left(c^{\prime}, d^{\prime} ; R^{\prime}\right)\right) .
$$

In view of (15.16) and (15.14a) the upper bound of

$$
w_{0}^{*}(\lambda, u)\left|e^{-G_{\beta}(\lambda, u)}\right| \quad\left(\lambda, u \text { in } \Gamma\left(c^{\prime}, d^{\prime} ; R^{\prime}\right)\right),
$$

for

$$
c^{\prime} \leqq x \leqq u \leqq d^{\prime},
$$

is attained at $x$; the function $w_{0}^{*}(\lambda, u)$, and hence

$$
\left(w_{0}^{*}(\lambda, u)\right)^{2}\left|e^{-G_{\beta}(\lambda, u)}\right|,
$$

will possess the same property. By (15.9) (for $k=1$ ), (15.18), and the stated property of (15.20)

$$
\left|w_{j: 1}(\lambda, x)\right|<\left(d^{\prime}-c^{\prime}\right) n^{2} \gamma^{2}|\lambda|^{p+\omega} b\left(w_{0}^{*}(\lambda, x)\right)^{2} .
$$

Thus, by (15.17)

$$
\left|w_{j: 1}(\lambda, x)\right|<\Gamma(\lambda, x) w_{0}(\lambda, x)=w_{1}^{*}(\lambda, x) \quad\left(\text { in } \Gamma\left(c^{\prime}, d^{\prime} ; R^{\prime}\right)\right) .
$$

Making use of (15.4) for $k=2$ (with $\rho_{j: 0}=w_{j: 0}$ ) and applying (15.1), (15.2), with

$$
\rho^{*}=\rho_{0}^{*}(\lambda, x)=w_{0}^{*}(\lambda, x), \quad w^{*}=w_{1}^{*}(\lambda, x) \dagger
$$

we infer that

$$
\begin{aligned}
& \left|g_{j: 2}(\lambda, x)\right|<\left(q^{\prime} w_{0}^{*}(\lambda, x)+q w_{1}^{*}(\lambda, x)\right) w_{1}^{*}(\lambda, x) \\
& \quad=\left[q^{\prime}+q \Gamma(\lambda, x)\right] w_{0}^{*}(\lambda, x) w_{1}^{*}(\lambda, x) \\
& \quad<\left[q^{\prime}+q \cdot\left(\frac{\rho^{\prime \prime}}{1+\rho^{\prime \prime}}\right)\right] w_{0}^{*}(\lambda, x) w_{1}^{*}(\lambda, x)<b w_{0}^{*}(\lambda, x) w_{1}^{*}(\lambda, x)
\end{aligned}
$$

(in $\left.\Gamma\left(c^{\prime}, d^{\prime} ; R^{\prime}\right)\right)$

Assume that for $\lambda, x$ in $\Gamma\left(c^{\prime}, d^{\prime} ; R^{\prime}\right)\left(|\lambda| \geqq r^{\prime}\right)$ and for $i=1, \cdots, k(\geqq 2)$ the following inequalities hold

$$
\left|g_{j: i}(\lambda, x)\right|<g_{i}=b w_{0}^{*}(\lambda, x) w_{i-1}^{*}(\lambda, x),
$$

$\dagger \rho^{*}+w^{*}=w_{0}^{*}+w_{1}^{*}<\left(\rho^{\prime \prime} /\left(1+\rho^{\prime \prime}\right)\right)+\left(\rho^{\prime \prime} /\left(1+\rho^{\prime \prime}\right)\right)^{2}<\rho^{\prime \prime}$ by $(15.17)$ and (15.21).

$\ddagger$ By definition of $b$. 


$$
\left|w_{j: i-1}(\lambda, x)\right|<w_{i-1}^{*}(\lambda, x)=\Gamma^{i-1}(\lambda, x) w_{0}^{*}(\lambda, x) \quad(j=1, \cdots, n) .
$$

For $k=2$ these relations have been established in (15.18), (15.22), (15.16), and (15.21).

In consequence of (15.23) for $i=k$, and (15.23a) for $i=k$

$$
g_{k}=g_{k}(\lambda, u)=b \Gamma^{k-1}(\lambda, u)\left(w_{0}^{*}(\lambda, u)\right)^{2} .
$$

In view of the property previously stated in connection with the function (15.19) and $w_{0}^{*}(\lambda, u)$, and in view of the fact that $\Gamma(\lambda, u)$ enjoys the same property, $\dagger$ if we write

(15.24a) $g_{k}(\lambda, u)\left|e^{-G_{\beta}(\lambda, u)}\right|=b \Gamma^{k-1}(\lambda, u) w_{0}^{*}(\lambda, u)\left[w_{0}^{*}(\lambda, u) e^{-G_{j}(\lambda, u)}\right]$,

we infer that the function on the left-hand side of (15.24a) will attain its upper bound at $u=x$, for $\lambda, u$ in $\Gamma\left(c^{\prime}, d^{\prime} ; R^{\prime}\right)$ (provided (15.19a) is satisfied). Hence from (15.9) it follows that

$$
\left|w_{j: k}(\lambda, x)\right|<n^{2} \gamma^{2}|\lambda|^{p+\omega}\left(d^{\prime}-c^{\prime}\right) g_{k}(\lambda, x)=k_{1}|\lambda|^{p+\omega} b \Gamma^{k-1}(\lambda, x)\left(w_{0}^{*}(\lambda, x)\right)^{2} .
$$

Thus, by (15.17)

$$
\begin{aligned}
&\left|w_{j: k}(\lambda, x)\right|<\Gamma^{k}(\lambda, x) w_{0}^{*}(\lambda, x)=w_{k}^{*}(\lambda, x) \\
& \quad\left(\text { in } \Gamma\left(c^{\prime}, d^{\prime} ; R^{\prime}\right) ; j=1, \cdots, n\right) .
\end{aligned}
$$

In other words, (15.23a) will hold for $i=1, \cdots, k+1$.

We are going to apply (15.4) with $k$ replaced by $k+1$. We may write

$$
\begin{aligned}
& \left|\rho_{j: k-1}(\lambda, x)\right|=\left|w_{j: 0}(\lambda, x)+\cdots+w_{j: k-1}(\lambda, x)\right|<w_{0}^{*}(\lambda, x)+\cdots \\
& \quad+w_{k-1}^{*}(\lambda, x)=\rho^{*}, \quad\left|w_{j: k}(\lambda, x)\right|<w_{k}^{*}(\lambda, x)=w^{*} \quad(j=1, \cdots, n) .
\end{aligned}
$$

Now, by (15.23a) $(i=1, \cdots, k+1)$ and by virtue of (15.17)

$$
\rho^{*}+w^{*}<\left(\frac{\rho^{\prime \prime}}{1+\rho^{\prime \prime}}\right)+\left(\frac{\rho^{\prime \prime}}{1+\rho^{\prime \prime}}\right)^{2}+\cdots+\left(\frac{\rho^{\prime \prime}}{1+\rho^{\prime \prime}}\right)^{k+1}<\rho^{\prime \prime} .
$$

Hence the statement in connection with (15.1), (15.2) is applicable. We have

$$
\begin{aligned}
& \left|g_{j: k+1}(\lambda, x)\right|<\left(q^{\prime} \rho^{*}+q w^{*}\right) w^{*} \\
& \quad=\left[q^{\prime}\left(1+\Gamma(\lambda, x)+\cdots+\Gamma^{k-1}(\lambda, x)\right)+q \Gamma^{k}(\lambda, x)\right] w_{0}(\lambda, x) w_{k}^{*}(\lambda, x) \\
& \quad<\left[q^{\prime}\left(1+\delta+\cdots+\delta^{k-1}\right)+q \delta^{k}\right] w_{0}^{*}(\lambda, x) w_{k}^{*}(\lambda, x) \\
& \quad \leqq b w_{0}^{*}(\lambda, x) w_{k}^{*}(\lambda, x)=g_{k+1} \quad\left(\delta=\rho^{\prime \prime} /\left(1+\rho^{\prime \prime}\right) ; \text { in } \Gamma\left(c^{\prime}, d^{\prime} ; R^{\prime}\right)\right) .
\end{aligned}
$$

Whence it is concluded that the inequalities (15.23), (15.23a) hold for $i=1,2, \cdots$. Consequently the series (15.5) converge absolutely and uniformly in $\Gamma\left(c^{\prime}, d^{\prime} ; R^{\prime}\right)\left(|\lambda| \geqq r^{\prime}\right)$; in fact, 


$$
\begin{aligned}
\left|\rho_{j}(\lambda, x)\right| \leqq & w_{j: 0}(\lambda, x)|+| w_{j: 1}(\lambda, x) \mid+\cdots \\
& <w_{0}^{*}(\lambda, x)+w_{1}^{*}(\lambda, x)+\cdots \\
& =w_{0}^{*}(\lambda, x)\left[1+\Gamma(\lambda, x)+\Gamma^{2}(\lambda, x)+\cdots\right] \\
\leqq & w_{0}^{*}(\lambda, x)\left[1+\delta+\delta^{2}+\cdots\right]=w_{0}^{*}(\lambda, x)\left(1+\rho^{\prime \prime}\right) \\
& \quad\left(\text { in } \Gamma\left(c^{\prime}, d^{\prime} ; R^{\prime}\right) ; j=1, \cdots, n\right) .
\end{aligned}
$$

The absolute values of the coefficients of $w_{1: i}(i=1, \cdots, n)$ in

$$
l_{j}^{*}\left(\lambda, x, w_{1: k}, \cdots, w_{n: k}\right)
$$

are $\leqq K$, where $K$ is independent of $\lambda$ and $x\left(\lambda, x\right.$ in $\left.\Gamma\left(c^{\prime}, d^{\prime} ; R^{\prime}\right)\right)$. By (15.4) for $k=0$

$$
\left|w_{j: 0}^{(1)}(\lambda, x)\right| \leqq|\lambda|^{p} n K w_{0}^{*}(\lambda, x)+|\lambda|^{p} g_{0}(\lambda, x) .
$$

Substituting the expression for $g_{0}(\lambda, x)$ from (15.16), we obtain

$$
\left|w_{j: 0}^{(1)}(\lambda, x)\right|<k^{\prime \prime}|\lambda|^{p} w_{0}^{*}(\lambda, x) \quad\left(j=1, \cdots, n ; \text { in } \Gamma\left(c^{\prime}, d^{\prime} ; R^{\prime}\right)\right) . \dagger
$$

By virtue of (15.4) for $k>1$, (15.25), and (15.24) and the fact that $\Gamma(\lambda, x)$, $w_{0}^{*}(\lambda, x) \leqq \delta$, it is seen that

$$
\begin{aligned}
\left|w_{j: k}^{(1)}(\lambda, x)\right| & \leqq|\lambda|^{p} n K w_{k}^{*}(\lambda, x)+|\lambda|^{p} b \Gamma^{k-1}(\lambda, x)\left(w_{0}^{*}(\lambda, x)\right)^{2} \\
& <(n K+b)|\lambda|^{p} \delta^{k} w_{0}^{*}(\lambda, x) .
\end{aligned}
$$

Thus, with ${ }_{1} k$ denoting the greater of the numbers $k^{\prime \prime}, n K+b$, we have

$$
\left|w_{j: k}^{(1)}(\lambda, x)\right|<{ }_{1} k|\lambda|^{p} \delta^{k} w_{0}^{*}(\lambda, x) \quad(k=0,1, \cdots ; j=1, \cdots, n)
$$

in $\Gamma\left(c^{\prime}, d^{\prime} ; R^{\prime}\right)$.

With the aid of (15.28) it is concluded that the series

$$
\rho_{j}^{(1)}(\lambda, x)=w_{j: 0}^{(1)}(\lambda, x)+w_{j: 1}^{(1)}(\lambda, x)+\cdots
$$

converge absolutely and uniformly. We have, for $\lambda, x$ in $\Gamma\left(c^{\prime}, d^{\prime} ; R^{\prime}\right)$,

$$
\begin{array}{r}
\left|\rho_{j}^{(1)}(\lambda, x)\right| \leqq\left|w_{j: 0}^{(1)}(\lambda, x)\right|+\cdots<\left(1+\rho^{\prime \prime}\right)_{1} k|\lambda|^{p} w_{0}^{*}(\lambda, x) \\
(j=1, \cdots, n) .
\end{array}
$$

For $\lambda, x$ in $\Gamma\left(c^{\prime}, d^{\prime} ; R^{\prime}\right)\left(|\lambda| \geqq r^{\prime} ; r^{\prime}\right.$ sufficiently great) the series (15.5) will truly represent a solution of the transformed system. The $\rho_{j}(\lambda, x)$ are analytic in $\lambda$ for $\lambda$ in $R^{\prime}(\lambda \neq \infty)$, provided $x$ is in $\left(c^{\prime}, d^{\prime}\right)$. For any fixed $\lambda$ in $R^{\prime}$ the $\rho_{j}(\lambda, x)$ will be continuous in $x$ for $x$ in $\left(c^{\prime}, d^{\prime}\right)$. $\ddagger$ Moreover, these functions are differentiable in $\Gamma\left(c^{\prime}, d^{\prime} ; R^{\prime}\right)$ with respect to $x$.

$\dagger k^{\prime \prime}$ is independent of $\lambda, x$.

$\ddagger$ The $\rho_{j}(\lambda, x)$ are uniformly convergent series of continuous functions. 
The Third Existence Theorem. Let $\Gamma\left(\alpha^{\prime}, \beta^{\prime} ; R\right)$ be a region proper according to Definition $2, \S 12$. The functions $Q_{i}(\lambda, x)$, here involved, are from the formal matrix solution (12.2) satisfying the linear problem (12.1) (associated with the system (11.1)). Let the $G_{i}(\lambda, x)(i=1, \cdots, n)$ be defined by (12.6b). Let $\Gamma\left(c, d ; R^{\prime}\right)$ be an admissible (cf. Definition $\left.3, \S 12\right)$ subregion of $\Gamma\left(\alpha^{\prime}, \beta^{\prime} ; R\right)$. With suitable notation we then have (12.10), (12.10a), (12.10b), and (12.10c).

Let $N(>0)$ be a fixed integer, however large. Form a region $\Gamma\left(c^{\prime}, d^{\prime} ; R^{\prime}\right)$, where $R^{\prime}$ is the same region as before and $\left(c^{\prime}, d^{\prime}\right)$ is a subinterval of $(c, d)$ such that the italicized statement preceding (13.17) should hold for $\left(c^{\prime}, d^{\prime}\right)$. Let the $c_{i}$ $(i=1, \cdots, m)$ be arbitrary functions of $\lambda$ (independent of $x)$, subject, however, to the condition stated in italics in connection with (14.2).

The system (11.1) will then possess a solution $y_{j}(\lambda, x)(j=1, \cdots, n)$, whose elements are analytic in $\lambda$ for $\lambda$ in $R^{\prime}(\lambda \neq \infty)\left(x\right.$ in $\left.\left(c^{\prime}, d^{\prime}\right)\right)$ and satisfy asymptotic relations

$$
y_{j}(\lambda, x) \sim s_{j}(\lambda, x) \quad\left(j=1, \cdots, n ; \text { in } \Gamma\left(c^{\prime}, d^{\prime} ; R^{\prime}\right)\right),
$$

where the $s_{j}(\lambda, x)$ are the series referred to in Lemma $2, \$ 13$. The relations (15.30) are asymptotic in the following sense. The $y_{j}(\lambda, x)$ are of the form

$$
\begin{aligned}
y_{j}(\lambda, x)=\sum_{\nu=1}^{N-1} \sum_{k_{1}+\cdots+k_{m}=\nu} c_{1}^{k_{1}} \cdots c_{m}^{k_{m}}{ }_{\nu} \eta_{k_{1}, \cdots, k_{m} ; j}(\lambda, x)+\rho_{j}(\lambda, x) \\
(j=1, \cdots, n),
\end{aligned}
$$

where

$$
\begin{array}{r}
{ }_{\nu \eta_{k_{1}}, \cdots, k_{m} ; j}(\lambda, x) \\
\quad=\exp \left[k_{1} G_{1}(\lambda, x)+\cdots+k_{m} G_{m}(\lambda, x)\right] \lambda^{(\nu-1)(p+\omega)}[\lambda, x]_{\bar{\alpha}-\nu+1} \\
\left(k_{1}, \cdots, k_{m} \geqq 0 ; k_{1}+\cdots+k_{m}=\nu ; \nu=1,2, \cdots, N-1 ; j=1, \cdots, n\right)
\end{array}
$$

for $\lambda, x$ in $\Gamma\left(c^{\prime}, d^{\prime} ; R^{\prime}\right)$. The symbol $[\lambda, x]_{\bar{\alpha}-\nu+1}$, above, is defined in $\Gamma\left(c^{\prime}, d^{\prime} ; R^{\prime}\right)$ according to Definition $4, \S 13$. The $\rho_{j}(\lambda, x)$ are functions defined, together with the derivatives $\rho_{j}^{(1)}(\lambda, x)$, in $\Gamma\left(c^{\prime}, d^{\prime} ; R^{\prime}\right)$ and are such that

$$
\begin{aligned}
& \left|\rho_{j}(\lambda, x)\right|<\bar{k} \gamma^{N}(\lambda)|\lambda|^{(N-1)(p+\omega)} \\
& \quad \sum_{h_{1}+\cdots+h_{m}=N}\left|\exp \left[h_{1} G_{1}(\lambda, x)+\cdots+h_{m} G_{m}(\lambda, x)\right]\right|=\rho_{N}^{*}(\lambda, x),
\end{aligned}
$$

where $\gamma(\lambda)$ is defined as in (14.2) and

$$
\left|\rho_{j}^{(1)}(\lambda, x)\right|<\bar{k}_{1} \rho_{N}^{*}(\lambda, x)|\lambda|^{p} \quad\left(j=1, \cdots, n ; \text { in } \Gamma\left(c^{\prime}, d^{\prime} ; R^{\prime}\right)\right),
$$

$\bar{k}, \bar{k}_{1}$ being independent of $\lambda, x$. In the above, $\bar{\alpha}$, depending on $N$, is to be taken 
suitably great. In $R^{\prime},|\lambda| \geqq r^{\prime}$ and $r^{\prime}$ is to be taken sufficiently great so that (14.4a) and (15.17) † will hold.

Note. We observe that $\left|\rho_{j}(\lambda, x)\right|$ is essentially of the order of

$$
\sum_{k_{1}+\cdots+k_{m}=N} c_{1}^{k_{1}} \cdots c_{m N \eta_{k_{1}}, \cdots, k_{m} ; j}^{k_{m}}(\lambda, x) \text {. }
$$

In fact, the absolute value of the latter expression is less than

$$
h \rho_{N}^{*}(\lambda, x) \quad\left(\text { in } \Gamma\left(c^{\prime}, d^{\prime} ; R^{\prime}\right)\right),
$$

where $h$ is independent of $\lambda, x$.

The following general remarks will be made regarding the main results of this work.

The remaining problem is of interest, namely, to determine under what specialized conditions the formal series solutions involved in the three existence theorems converge. It is to be noted that in all of Horn's work, which relates to certain restricted first-order problems, $\ddagger$ the formal solutions converge and thus represent actual solutions. $\S$

Another problem of importance is to find under what restrictions are the coefficients in the formal series solutions (in the case of the First Existence Theorem) representable with the aid of the fundamental methods of Nörlund (Laplace integrals leadings to convergent factorial series developments-essentially by a method of exponential summability).\|

Finally, it is observed that the existence theorems of this work can be extended without any substantial difficulties by replacing the second members in the systems (A), (B), and (C) of $\$ 1$ by suitable functions satisfying in a certain neighborhood of the singular point appropriate relations asymptotic with respect to $y_{1}, \cdots, y_{n}$ (to a finite number of terms with respect to $t$ $($ or $\lambda))$.

$\dagger$ These conditions can be also satisfied by other means (cf. the text in connection with (14.4a) and (15.17)).

$\ddagger$ For references to J. Horn see $\left(\mathrm{T}_{1}\right)$; also see my paper in Compositio Mathematica, loc. cit.

$\S$ Even in the first-order problems occasions arise when the formal solutions diverge. So even for these problems one is forced to use asymptotic methods, unless suitable restrictions are introduced.

\| Whenever applicable, these methods yield results of greater precision than those obtained by asymptotic methods. On the other hand, as indicated in $\left(T_{1}\right)$, they are applicable only under suitable restrictions.

UNIVERSITY OF ILLINOIS, URBANA, ILL. 